NBER WORKING PAPER SERIES

\title{
STRUCTURAL EQUATIONS, TREATMENT EFFECTS AND ECONOMETRIC POLICY EVALUATION
}

\author{
James J. Heckman \\ Edward Vytlacil \\ Working Paper 11259 \\ http://www.nber.org/papers/w11259 \\ NATIONAL BUREAU OF ECONOMIC RESEARCH \\ 1050 Massachusetts Avenue \\ Cambridge, MA 02138 \\ March 2005
}

This paper was presented by Heckman as the Fisher-Schultz Lecture at the Eighth World Meetings of the Econometric Society, Seattle, Washington, August 13, 2000. This paper was also presented at the seminar on Applied Price Theory at the Graduate School of Business, University of Chicago in October 2000, at a seminar at Upsalla University, December 2000, at Harvard University, April 2001, and at the Montreal Econometrics Seminar, September 2003. We thank Jaap Abbring, Richard Blundell and two anonymous referees for helpful comments on the first round reports. We benefited from the close reading of Ricardo Avelino, Jean-Marc Robin, Sergio Urzua, and Weerachart Kilenthong on the second draft. We have benefited from a close reading by Jora Stixrud and Sergio Urzua on the third draft. We have also benefited from comments by an anonymous referee on the second draft of this paper. Sergio Urzua provided valuable research assistance in programming the simulations reported in this paper and was assisted by Hanna Lee. Urzua made valuable contributions to our understanding of the random coefficient case and cases with negative weights and made numerous valuable comments on this draft, as did Weerachart Kilenthong. See our companion paper (Heckman, Urzua, and Vytlacil, 2004) where these topics are developed further. This research was supported by NSF 97-09-873, NSF-00-99195, NSF SES-0241858, and NIH R01 HD043411, and the American Bar Foundation. The views expressed herein are those of the author(s) and do not necessarily reflect the views of the National Bureau of Economic Research.

(C2005 by James J. Heckman and Edward Vytlacil. All rights reserved. Short sections of text, not to exceed two paragraphs, may be quoted without explicit permission provided that full credit, including $(\odot)$ notice, is given to the source. 
Structural Equations, Treatment Effects and Econometric Policy Evaluation

James J. Heckman and Edward Vytlacil

NBER Working Paper No. 11259

March 2005

JEL No. C1

\begin{abstract}
This paper uses the marginal treatment effect (MTE) to unify the nonparametric literature on treatment effects with the econometric literature on structural estimation using a nonparametric analog of a policy invariant parameter; to generate a variety of treatment effects from a common semiparametric functional form; to organize the literature on alternative estimators; and to explore what policy questions commonly used estimators in the treatment effect literature answer. A fundamental asymmetry intrinsic to the method of instrumental variables is noted. Recent advances in IV estimation allow for heterogeneity in responses but not in choices, and the method breaks down when both choice and response equations are heterogeneous in a general way.

James J. Heckman

Department of Economics

The University of Chicago

1126 E. 59th Street

Chicago, IL 60637

and NBER

j-heckman@uchicago.edu

Edward Vytlacil

Stanford University

vytlacil@stanford.edu
\end{abstract}


Evaluating the impacts of public policies, forecasting their effects in new environments, and predicting the effects of policies never tried are three central tasks of economics. The structural approach and the treatment effect approach are two competing paradigms of policy evaluation.

The structural approach emphasizes clearly articulated economic models that can be used to accomplish all three tasks under the exogeneity and parameter policy invariance assumptions presented in that literature. (See Hansen and Sargent, 1981; Hendry, 1995.) Economic theory is used to guide the construction of models and to suggest included and excluded variables. The functional form and exogeneity assumptions invoked in this literature are sometimes controversial (see, e.g., Angrist and Krueger, 1999) and the sources of identification of parameters of these models are often not clearly articulated.

The treatment effect literature as currently developed focuses on the first task-evaluating the impact of a policy in place - in the special case where there is a "treatment group" and a "comparison group," i.e. a group of nonparticipants. In the language of that literature, "internal validity" is the primary goal and issues of forecasting out of sample or of evaluating new policies receive little attention. ${ }^{1}$ Because of its more limited goals, fewer explicit functional form and exogeneity assumptions are invoked. The literature on treatment effects has given rise to a new language of economic policy analysis where the link to economic theory is often obscure and the economic policy questions being addressed are not always clearly stated. Different instruments answer different economic questions that typically are not clearly stated. Relationships among the policy parameters implicitly defined by alternative choices of instruments are not articulated.

This paper unites the two approaches to policy evaluation using the Marginal Treatment Effect (MTE) under the assumption that analysts have access to treatment and comparison groups. The MTE is the mean response of persons to treatment at a margin that is precisely defined in this paper. It is a willingness to pay measure when outcomes are values under alternative treatment regimes.

Under the conditions specified in this paper, the MTE can be used to construct and compare alternative conventional treatment effects, a new class of policy relevant treatment effects and the probability limits produced from instrumental variable estimators and matching estimators. Using the MTE, this paper unites the selection (control function) approach, defined in a nonparametric setting, with the recent

\footnotetext{
${ }^{1}$ Internal validity means that a treatment parameter defined in a specified environment is free of selection bias. It is defined more precisely below.
} 
literature on instrumental variables.

A major focus in the recent microeconomic policy evaluation literature, and a major theme of this paper, is on constructing and estimating models with heterogeneity in responses to treatment among otherwise observationally identical people. This literature emphasizes that responses to treatment vary among observationally identical people, and crucially, that agents select (or are selected) into treatment at least in part based on their own idiosyncratic response to it. This emphasis is in marked contrast to the emphasis in the conventional representative-agent macro time series literature that ignores such heterogeneity despite ample microeconometric evidence on it. ${ }^{2}$

Entire classes of econometric evaluation estimators can be organized by whether or not they allow for the possibility of selection based on unobserved components of heterogeneous responses to treatment. In the presence of such heterogeneity, a variety of different mean treatment effects can be defined for different instruments and conditioning sets. In the absence of such heterogeneity, these different treatment effects collapse to the same parameter. ${ }^{3}$

The dependence of estimated treatment parameters on instruments is an important and not widely understood feature of models with heterogeneous responses on which people act. ${ }^{4}$ Instrument-dependent parameters arise in this class of models, something excluded by assumption in conventional structural econometric models that emphasize the estimation of invariant parameters. Two economists analyzing the same data set but using different valid instruments will estimate different parameters that have different economic interpretations. Even more remarkably, two economists using the same instrument but with different notions about what variables belong in choice equations will interpret the output of an instrumental variable analysis differently. Intuitions about "identifying strategies" acquired from analyzing conventional models where responses to treatment do not vary among persons are not valid in the more general setting analyzed in this paper. The choice of an instrument defines the treatment parameter being estimated. The relevant question regarding the choice of instrumental variables in the general class of models studied in this paper is "What parameter is being identified by the instrument?" rather than the traditional question of "What is the efficient combination of instruments for a fixed parameter?" - the question that

\footnotetext{
${ }^{2}$ Heckman (2001) summarizes the evidence on heterogeneity in responses to treatment on which agents select into treatment.

${ }^{3}$ See Heckman (1997); Heckman and Robb (1985, 1986); Heckman and Vytlacil (1999).

${ }^{4}$ This dependence was first noted by Heckman and Robb (1985, p. 196). See also Angrist, Graddy, and Imbens (2000).
} 
has traditionally occupied the attention of econometricians who study instrumental variables. Even in the presence of least squares bias, and even assuming large samples, $I V$ based on classical assumptions may be more biased for a given policy parameter than $O L S$. The cure may be worse than the disease.

We extend the method of instrumental variables to estimate economically interpretable parameters in models with heterogeneous treatment outcomes. We note a fundamental asymmetry intrinsic to the method of instrumental variables. Treatment outcomes can be heterogeneous in a general way that we make precise in this paper. Choice equations cannot be heterogeneous in the same general way. When choices and treatment outcomes are analyzed symmetrically, the method of instrumental variables and our extension of it breaks down, and more explicit structural approaches are necessary to solve policy evaluation problems.

The plan of this paper is as follows. Section 1 presents a prototypical microeconometric structural model as a benchmark to define and motivate the various treatment parameters used in the literature and to compare and contrast structural estimation approaches with those used in the literature on treatment effects. We then define our general model and assumptions in Section 2. Our model extends the treatment effect literature by introducing choice theory into it and by using a weaker set of assumptions than those used in the structural literature to define and identify the marginal treatment effect $(M T E)$. This section shows how the MTE can be used to generate and unify the various treatment parameters advocated in the recent literature and provides an economic foundation for the treatment effect literature. We derive a set of testable restrictions implied by our model, and we apply the general analysis to the special case of a parametric Generalized Roy Model.

The conventional treatment parameters do not, in general, answer questions of economic or policy interest. Section 3 shows how to use the MTE to define policy relevant parameters that answer well posed economic questions. Evaluation of different policies requires different weights for the MTE. The MTE plays the role of a policy invariant structural parameter in conventional econometrics for a class of policy interventions defined in this paper. ${ }^{5}$

Section 4 organizes entire classes of econometric estimators on the basis of what they assume about the role of unobservables in the MTE function, conditional on $X$. Our analysis shows that traditional instrumental variables procedures require that the marginal treatment effect is the same for all persons

\footnotetext{
${ }^{5}$ Hendry (1995) discusses the role of policy invariant parameters in macro-forecasting and policy evaluation.
} 
of given $X$ characteristics. When the marginal treatment effect varies over individuals with the same $X$, we show how the instrumental variables estimand (the probability limit of the instrumental variables estimator) can be written as a weighted average of $M T E$, where our general expressions nest previous results in the literature as special cases. The interpretation of the $I V$ estimand depends not only on the choice of instrument used but also on what other variables are included in the choice model even if they are not used as instruments. We show that it is not always possible to pick an instrument that answers a particular policy problem of interest, and we show that not all instruments answer well defined policy questions. We present necessary and sufficient conditions to construct an instrument to produce a particular policy counterfactual, and show how to construct the instrument when the conditions are satisfied. We develop necessary and sufficient conditions for a particular instrument to answer some well defined policy question, and show how to construct the policy counterfactual when the conditions are satisfied. We focus on instrumental variables in this paper but also consider matching and ordinary least squares as special cases of our general model for $I V$.

Section 5 returns to the policy evaluation problem. The treatment effect literature can be used to answer certain narrowly focused questions under weaker assumptions than are required to recover conventional structural parameters that answer a broad range of questions. When we attempt to address the broader set of questions entertained in the structural econometrics literature, additional conditions are required to extrapolate existing policies to new environments and to provide accurate forecasts of new policies never previously experienced. The weaker identifying assumptions invoked in the treatment effect literature are possible because of the narrower set of questions addressed by that literature. In the language of the treatment effect literature, internal validity (absence of selection bias) does not imply external validity (the ability to generalize). When the same policy forecasting questions addressed by the structural literature are asked of the treatment effect literature, the assumption sets used in the two literatures look very similar, especially for nonparametric versions of structural models. External validity requires stronger conditions.

Section 6 discusses the fundamental role played by the assumed absence of general forms of heterogeneity in choice equations invoked in the recent literature under the rubric of "monotonicity" assumptions. When both choices and treatment outcomes are modelled symmetrically, the method of instrumental variables breaks down, and a different approach to policy analysis is required. Section 7 concludes. 


\section{A Latent Variable Framework}

The treatment effect literature investigates a class of policies that have partial participation at a point in time so there is a "treatment" group and a "comparison" group. It is not helpful in evaluating policies that have universal participation. In contrast, the structural econometrics literature can evaluate policies with universal participation by using functional form and support conditions to substitute for lack of a comparison group (see Heckman and Vytlacil, 2005). Throughout this paper we follow the conventional practice in the literature and ignore general equilibrium effects. ${ }^{6}$

In order to link our discussion to the literature on structural econometrics, it is fruitful to compare how the two different approaches analyze a Generalized Roy Model for two potential outcomes $\left(Y_{0}, Y_{1}\right)$. This model is widely used in applied econometrics (see Amemiya, 1985, and Heckman, 2001).

Write potential outcomes $\left(Y_{0}, Y_{1}\right)$ for conditioning variables $X$ as

$$
Y_{0}=\mu_{0}(X)+U_{0}
$$

and

$$
Y_{1}=\mu_{1}(X)+U_{1}
$$

where $Y_{1}$ is the outcome if treated and $Y_{0}$ is the outcome if not treated. ${ }^{7}$ In a model of educational attainment, $Y_{1}$ is the present value of college earnings and $Y_{0}$ is the present value of earnings in the benchmark no-treatment state (e.g. high school). Let $D=1$ denote receipt of treatment so that $Y_{1}$ is observed, while $D=0$ denotes that treatment was not received so that $Y_{0}$ is observed. In the educational attainment example, $D=1$ if the individual selects into college, $D=0$ otherwise. The observed outcome $Y$ is given by

$$
Y=D Y_{1}+(1-D) Y_{0}
$$

Let

$$
C=\mu_{C}(Z)+U_{C}
$$

\footnotetext{
${ }^{6}$ See, however, the studies by Heckman, Lochner, and Taber (1998), who demonstrate the empirical importance of investigating general equilibrium effects in the context of evaluating the returns to schooling.

${ }^{7}$ Throughout this paper, we denote random variables/random vectors by capital letters and potential realizations by the corresponding lower case letter. For example, $X$ denotes the random vector, and $x$ denotes a potential realization of the random vector $X$.
} 
denote the cost of receiving treatment. Net utility is $D^{*}=Y_{1}-Y_{0}-C$ and the agent selects into treatment if the net utility from doing so is positive, $D=\mathbf{1}\left[D^{*} \geq 0\right]$.

The original Roy Model (1951) is a special case of this framework when there are zero costs of treatment, $\mu_{C}(Z)=0$ and $U_{C}=0$. The generalized Roy model allows for costs of treatment, both driven by observable determinants of the cost of treatment, $Z$, and unobservable determinants of the cost of treatment, $U_{C}$. For example, in the educational attainment example, tuition and family income operate through direct costs $\mu_{C}(Z)$ to determine college attendance, while $U_{C}$ might include disutility from studying. The model can be generalized to incorporate uncertainty about the benefits and costs of treatment and to allow for more general decision rules. Let $\mathcal{I}$ denote the information set available to the agent at the time when the agent is deciding whether to select into treatment. If, for example, the agent selects into treatment when the expected benefit exceeds the expected cost, then the index is $D^{*}=E\left(Y_{1}-Y_{0}-C \mid \mathcal{I}\right)$. The decision to participate is based on $\mathcal{I}$ and $D=\mathbf{1}\left[D^{*} \geq 0\right]$ where $D^{*}$ is a random variable measurable with respect to $\mathcal{I}^{8}$

Conventional approaches used in the structural econometrics literature assume that $(X, Z) \Perp\left(U_{0}, U_{1}, U_{C}\right)$, where " $\Perp$ " denotes independence. In addition, they adopt parametric assumptions about the distributions of the error terms and functional forms of the estimating equations, and identify the full model that can then be used to construct a variety of policy counterfactuals. The most commonly used specification of this model writes $\mu_{0}(X)=X \beta_{0}, \mu_{1}(X)=X \beta_{1}, \mu_{C}(Z)=Z \beta_{C}$ and assumes $\left(U_{0}, U_{1}, U_{C}\right) \sim N(0, \Sigma)$. This is the normal selection model (Heckman, 1976).

The parametric normal framework can be used to answer all three policy evaluation questions. First, it can be used to evaluate existing policies by asking how policy-induced changes in $X$ or $Z$ affect $(Y, D)$. Second, it can be used to extrapolate old policies to new environments by computing outcomes for the values of $X, Z$ that characterize the new environment. Linearity and distributional assumptions make extrapolation straightforward. Third, this framework can be used to evaluate new policies if they can be expressed as some known functions of $(X, Z)$. For example, consider the effect of charging tuition in an environment where tuition has never before been charged. If tuition can be put on the same footing as (made comparable with) another measure of cost that is measured and varies, or with returns that can be

\footnotetext{
${ }^{8}$ See Cunha, Heckman, and Navarro (2005) for a version of this model.
} 
measured and vary, then we can use the estimated response to the variation in observed costs or returns to estimate the response to the new tuition policy. ${ }^{9}$

This paper relaxes the functional form and distributional assumptions used in the structural literature and still identifies an economically interpretable model that can be used for policy analysis. Recent semiparametric approaches relax both distributional and functional form assumptions of selection models, but typically assume exogeneity of $X$ (see, e.g., Powell, 1994) and do not estimate treatment effects except through limit arguments (Heckman, 1990; Andrews and Schafgans, 1998). ${ }^{10}$ The treatment effect literature seeks to bypass the ad hoc assumptions used in the structural literature and estimate treatment effects under weaker conditions. The goal of this literature is to examine the effects of policies in place (i.e. to produce internally valid estimators) rather than to forecast new policies or old policies on new populations.

\section{Treatment Effects}

We now present the model of treatment effects developed in Heckman and Vytlacil (1999) and Heckman, Urzua, and Vytlacil (2004), which relaxes most of the controversial assumptions discussed in Section 1 . It is a nonparametric selection model with testable restrictions that can be used to unify the treatment effect literature, identify different treatment effects, link the literature on treatment effects to the literature in structural econometrics and interpret the implicit economic assumptions underlying instrumental variables and matching methods. We follow Heckman and Vytlacil (1999) and Heckman, Urzua, and Vytlacil (2004) in considering binary treatments. Heckman and Vytlacil (2005) and Heckman, Urzua, and Vytlacil (2004) extend this analysis to the case of a discrete, multi-valued treatment, for both ordered and unordered models, while Florens, Heckman, Meghir, and Vytlacil (2002) develop a related model with a continuum of treatments.

We use the general framework of Section 1, equations (1a)-(1d), and define $Y$ as the measured outcome

\footnotetext{
${ }^{9}$ For example, in a present value income maximizing model of schooling, costs and returns are on the same footing so knowledge of how schooling responds to returns is enough to determine how schooling responds to costs. See Section 5.1.

${ }^{10} \mathrm{~A}$ large part of the literature is concerned with estimation of slope coefficients (e.g., Ahn and Powell, 1993) and not the counterfactuals needed for policy analysis. Heckman (1990) develops the more demanding conditions required to identify policy counterfactuals.
} 
variable. We do not impose any assumption on the support of the distribution of $Y$. We use the more general nonlinear and nonseparable outcome model

$$
\begin{aligned}
& Y_{1}=\mu_{1}\left(X, U_{1}\right) \\
& Y_{0}=\mu_{0}\left(X, U_{0}\right) .
\end{aligned}
$$

Examples include conventional latent variable models: $Y_{i}=1$ if $Y_{i}^{*}=\mu_{i}(X)+U_{i} \geq 0$ and $Y_{i}=0$ otherwise; $i=0,1$. Notice that in the general case, $\mu_{i}\left(X, U_{i}\right)-E\left(Y_{i} \mid X\right) \neq U_{i}, i=0,1$, so even if the $\mu_{i}$ are structural, the $E\left(Y_{i} \mid X\right)$ are not. ${ }^{11}$

The individual treatment effect associated with moving an otherwise identical person from " 0 " to " 1 " is $Y_{1}-Y_{0}=\Delta$ and is defined as the effect on $Y$ of a ceteris paribus move from "0" to "1". These ceteris paribus effects are called "causal effects." To link this framework to the literature on structural econometrics, we characterize the decision rule for program participation by an index model:

$$
D^{*}=\mu_{D}(Z)-U_{D} ; \quad D=1 \quad \text { if } \quad D^{*} \geq 0 ; \quad D=0 \quad \text { otherwise }
$$

where $(Z, X)$ is observed and $\left(U_{1}, U_{0}, U_{D}\right)$ is unobserved. The random variable $U_{D}$ may be a function of $\left(U_{0}, U_{1}\right)$. For example, in the Roy Model $U_{D}=U_{1}-U_{0}$, and in the Generalized Roy Model $U_{D}=$ $U_{1}-U_{0}-U_{C}$. Without loss of generality, $Z$ includes all of the elements of $X$. However, our analysis requires that $Z$ contain at least one element not in $X$. The following assumptions are weaker than those used in the conventional literature on structural econometrics or the recent literature on semiparametric selection models and at the same time can be used to both define and identify different treatment parameters. ${ }^{12}$ The assumptions are:

$(\mathrm{A}-1) \mu_{D}(Z)$ is a nondegenerate random variable conditional on $X$;

(A-2) The random vectors $\left(U_{1}, U_{D}\right)$ and $\left(U_{0}, U_{D}\right)$ are independent of $Z$ conditional on $X$;

(A-3) The distribution of $U_{D}$ is absolutely continuous with respect to Lebesgue measure;

\footnotetext{
${ }^{11}$ See Heckman and Vytlacil (2005) for alternative definitions of structure.

${ }^{12}$ As noted in Section 2, and Heckman and Vytlacil (2001b), a much weaker set of conditions is required to define the parameters than is required to identify them. As noted in Section 5, stronger conditions are required for policy forecasting.
} 
(A-4) The values of $E\left|Y_{1}\right|$ and $E\left|Y_{0}\right|$ are finite; and

$(\mathrm{A}-5) 1>\operatorname{Pr}(D=1 \mid X)>0$.

Assumptions (A-1) and (A-2) are "instrumental variable" assumptions that there is at least one variable that determines participation in the program that is not in $X$ and that is independent of potential outcomes $\left(Y_{0}, Y_{1}\right)$ given $X$. These are the assumptions used in the natural and social experiment literatures where randomization or pseudorandomization generates instruments. (A-2) also assumes that $U_{D}$ is independent of $Z$ given $X$ and is used below to generate counterfactuals. Assumption (A-3) is a technical assumption made primarily for expositional convenience. Assumption (A-4) guarantees that the conventional treatment parameters are well defined. Assumption (A-5) is the assumption in the population of both a treatment and a control group for each $X$. Observe that there are no exogeneity requirements for $X$. This is in contrast with the assumptions commonly made in the conventional structural literature and the semiparametric selection literature (see, e.g., Powell, 1994). A counterfactual "no feedback" condition facilitates interpretability so that conditioning on $X$ does not mask the effects of $D$. Letting $X_{d}$ denote a value of $X$ if $D$ is set to $d$, a sufficient condition that rules out feedback from $D$ to $X$ is:

(A-6) $X_{1}=X_{0}$ almost everywhere.

Condition (A-6) is not strictly required to formulate an evaluation model, but it enables an analyst who conditions on $X$ to capture the "total" or "full effect" of $D$ on $Y$ (see Pearl, 2000). This assumption imposes the requirement that $X$ is an external variable determined outside the model and is not affected by counterfactual manipulations of $D$. However, the assumption allows for $X$ to be freely correlated with $U_{1}, U_{0}$ and $U_{D}$ so it can be endogenous in this sense. In this paper, we examine treatment effects conditional on $X$, and we maintain assumption (A-6) in this paper.

Define $P(Z)$ as the probability of receiving treatment given $Z: P(Z) \equiv \operatorname{Pr}(D=1 \mid Z)=F_{U_{D} \mid X}\left(\mu_{D}(Z)\right)$, where $F_{U_{D} \mid X}(\cdot)$ denotes the distribution of $U_{D}$ conditional on $X .{ }^{13}$ We often denote $P(Z)$ by $P$, suppressing the $Z$ argument. As a normalization, we impose $U_{D} \sim \operatorname{Unif}[0,1]$ and $\mu_{D}(Z)=P(Z)$. This normalization is innocuous given our assumptions, because if the latent variable generating choices is $D^{*}=\nu(Z)-V$,

\footnotetext{
${ }^{13}$ Throughout this paper, we will refer to the cumulative distribution function of a random vector $A$ by $F_{A}(\cdot)$ and to the cumulative distribution function of a random vector $A$ conditional on random vector $B$ by $F_{A \mid B}(\cdot)$. We will write the cumulative distribution function of $A$ conditional on $B=b$ by $F_{A \mid B}(\cdot \mid b)$.
} 
where $V$ is a general continuous random variable, we can apply a probability transform to reparameterize the model so that $\mu_{D}(Z)=F_{V \mid X}(\nu(Z))$ and $U_{D}=F_{V \mid X}(V){ }^{14}$

Vytlacil (2002) establishes that assumptions (A-1)-(A-5) for selection model (2a), (2b) and (3) are equivalent to the assumptions used to generate the LATE model of Imbens and Angrist (1994). Thus the nonparametric selection model for treatment effects developed in this paper is equivalent to an influential instrumental variable model for treatment effects. Our latent variable model satisfies their assumptions and their assumptions generate our latent variable model. Our latent variable model is a version of the standard sample selection bias model.

Our model and assumptions (A-1)-(A-5) impose two testable restrictions on the distribution of $(Y, D$, $Z, X)$. First it imposes an index sufficiency restriction: for any measurable set $\mathcal{A}$ and for $j=0,1$,

$$
\operatorname{Pr}\left(Y_{j} \in \mathcal{A} \mid X, Z, D=j\right)=\operatorname{Pr}\left(Y_{j} \in \mathcal{A} \mid X, P(Z), D=j\right)
$$

This restriction has empirical content when $Z$ contains two or more variables not in $X$. Second, the model also imposes a testable monotonicity restriction in $P=p$ for $E(Y D \mid X=x, P=p)$ and $E(Y(1-D) \mid X=x, P=p)$ which we develop in Appendix A.

Even though the model of treatment effects developed in this paper is not the most general possible model, it has testable implications and hence empirical content. It unites various literatures and produces a nonparametric version of the widely used selection model, and links the treatment literature to economic choice theory.

\section{Definitions of Treatment Effects}

The difficulty of observing the same individual in both treated and untreated states leads to the use of various population level treatment effects widely used in the biostatistics literature and applied in economics. ${ }^{15}$ The most commonly invoked treatment effect is the Average Treatment Effect $(A T E)$ :

\footnotetext{
${ }^{14}$ This representation is valid whether or not (A-2) is true. However, (A-2) imposes restrictions on counterfactual choices. For example, if a change in government policy changes the distribution of $Z$ by an external manipulation, under (A-2) the model can be used to generate the choice probability from $P(z)$ evaluated at the new arguments i.e., the model is invariant with respect to the distribution $Z$.

${ }^{15}$ Heckman, LaLonde, and Smith (1999) discussed panel data cases where it is possible to observe both $Y_{0}$ and $Y_{1}$ for the same person.
} 
$\Delta^{A T E}(x) \equiv E(\Delta \mid X=x)$ where $\Delta=Y_{1}-Y_{0}$. This is the effect of assigning treatment randomly to everyone of type $X$ assuming full compliance, and ignoring general equilibrium effects. The average impact of treatment on persons who actually take the treatment is Treatment on the Treated $(T T): \Delta^{T T}(x) \equiv E(\Delta \mid X=x, D=1)$. This parameter can also be defined conditional on $P(Z)$ : $\Delta^{T T}(x, p) \equiv E(\Delta \mid X=x, P(Z)=p, D=1){ }^{16}$

The mean effect of treatment on those for whom $X=x$ and $U_{D}=u_{D}$, the Marginal Treatment Effect $(M T E)$, plays a fundamental role in our analysis:

$$
\Delta^{M T E}\left(x, u_{D}\right) \equiv E\left(\Delta \mid X=x, U_{D}=u_{D}\right)
$$

The MTE is the expected effect of treatment conditional on observed characteristics $X$ and conditional on $U_{D}$, the unobservables from the first stage decision rule. For $u_{D}$ evaluation points close to zero, $\Delta^{M T E}\left(x, u_{D}\right)$ is the expected effect of treatment on individuals with the value of unobservables that make them most likely to participate in treatment and who would participate even if the mean scale utility $\mu_{D}(Z)$ were small. If $U_{D}$ is large, $\mu_{D}(Z)$ would have to be large to induce people to participate.

One can also interpret $E\left(\Delta \mid X=x, U_{D}=u_{D}\right)$ as the mean gain in terms of $Y_{1}-Y_{0}$ for persons with observed characteristics $X$ who would be indifferent between treatment or not if they were exogenously assigned a value of $Z$, say $z$, such that $\mu_{D}(z)=u_{d}$. When $Y_{1}$ and $Y_{0}$ are value outcomes, MTE is a mean willingness to pay measure. MTE is a choice-theoretic building block that unites the treatment effect, selection and matching literatures.

A third interpretation is that $M T E$ conditions on $X$ and the residual defined by subtracting the expectation of $D^{*}$ from $D^{*}: \tilde{U}_{D}=D^{*}-E\left(D^{*} \mid Z, X\right)$. These three interpretations are equivalent under separability in $D^{*}$, i.e., when (3) characterizes the choice equation, but lead to three different definitions of $M T E$ when a more general nonseparable model is developed. This point is developed further in Section 6.

The LATE parameter of Imbens and Angrist (1994) is a version of MTE. Define $D_{z}$ as a counterfactual choice variable, with $D_{z}=1$ if $D$ would have been chosen if $Z$ had been set to $z$, and $D_{z}=0$ otherwise. Let $\mathcal{Z}(x)$ denote the support of the distribution of $Z$ conditional on $X=x$. For any $\left(z, z^{\prime}\right) \in \mathcal{Z}(x) \times \mathcal{Z}(x)$ such that $P(z)>P\left(z^{\prime}\right), \operatorname{LATE}$ is $E\left(\Delta \mid X=x, D_{z}=1, D_{z^{\prime}}=0\right)=E\left(Y_{1}-Y_{0} \mid X=x, D_{z}=1, D_{z^{\prime}}=0\right)$,

\footnotetext{
${ }^{16}$ These two definitions of treatment on the treated are related by integrating out the conditioning $p$ variable: $\Delta^{T T}(x)=$ $\int_{0}^{1} \Delta^{T T}(x, p) d F_{P(Z) \mid X, D}(p \mid x, 1)$ where $F_{P(Z) \mid X, D}(\cdot \mid x, 1)$ is the distribution of $P(Z)$ given $X=x$ and $D=1$.
} 
the mean gain to persons who would be induced to switch from $D=0$ to $D=1$ if $Z$ were manipulated externally from $z^{\prime}$ to $z$. From the latent index model, it follows that LATE can be written as

$$
E\left(Y_{1}-Y_{0} \mid X=x, D_{z}=1, D_{z^{\prime}}=0\right)=E\left(Y_{1}-Y_{0} \mid X=x, u_{D}^{\prime} \leq U_{D}<u_{D}\right)=\Delta^{L A T E}\left(x, u_{D}, u_{D}^{\prime}\right)
$$

for $u_{D}=\operatorname{Pr}\left(D_{z}=1\right)=P(z), u_{D}^{\prime}=\operatorname{Pr}\left(D_{z^{\prime}}=1\right)=P\left(z^{\prime}\right)$, where assumption (A-2) implies that $\operatorname{Pr}\left(D_{z}=\right.$ $1)=\operatorname{Pr}(D=1 \mid Z=z)$ and $\operatorname{Pr}\left(D_{z^{\prime}}=1\right)=\operatorname{Pr}\left(D=1 \mid Z=z^{\prime}\right)$. Imbens and Angrist define the LATE parameter as the probability limit of an estimator. Their analysis conflates issues of definition of parameters with issues of identification. Our representation of LATE allows us to separate these two conceptually distinct matters and to define the LATE parameter more generally. One can imagine evaluating the right hand side of this equation at any $u_{D}, u_{D}^{\prime}$ points in the unit interval and not only at points in the support of the distribution of the propensity score $P(Z)$ conditional on $X=x$ where it is identified. From assumptions (A-2), (A-3), and (A-4), $\Delta^{L A T E}\left(x, u_{D}, u_{D}^{\prime}\right)$ is continuous in $u_{D}$ and $u_{D}^{\prime}$ and $\lim _{u_{D}^{\prime} \uparrow u_{D}} \Delta^{\operatorname{LATE}}\left(x, u_{D}, u_{D}^{\prime}\right)=\Delta^{M T E}\left(x, u_{D}\right) \cdot{ }^{17}$

Heckman and Vytlacil (1999) use assumptions (A-1)-(A-5) and the latent index structure to develop the relationship between $M T E$ and the various treatment effect parameters shown in the first three lines of Table IA. For example, in that table $\Delta^{T T}(x)$ is a weighted average of $\Delta^{M T E}$ :

$$
\Delta^{T T}(x)=\int_{0}^{1} \Delta^{M T E}\left(x, u_{D}\right) h_{T T}\left(x, u_{D}\right) d u_{D}
$$

where

$$
h_{T T}\left(x, u_{D}\right)=\frac{1-F_{P \mid X}\left(u_{D} \mid x\right)}{\int_{0}^{1}\left(1-F_{P \mid X}(t \mid x)\right) d t}=\frac{S_{P \mid X}\left(u_{D} \mid x\right)}{E(P(Z) \mid X=x)},
$$

and $S_{P \mid X}\left(u_{D} \mid x\right)$ is $\operatorname{Pr}\left(P(Z)>u_{D} \mid X=x\right)$ and $h_{T T}\left(x, u_{D}\right)$ is a weighted distribution (See Heckman and Vytlacil, 2001b). The parameter $\Delta^{T T}(x)$ oversamples $\Delta^{M T E}\left(x, u_{D}\right)$ for those individuals with low values of $u_{D}$ that make them more likely to participate in the program being evaluated. Treatment on the untreated (TUT) is defined symmetrically with TT and oversamples those least likely to participate. The

\footnotetext{
${ }^{17}$ This follows from Lebesgue's theorem for the derivative of an integral and holds almost everywhere with respect to Lebesgue measure. The ideas of the marginal treatment effect and the limit form of LATE were first introduced in the context of a parametric normal Generalized Roy model by Björklund and Moffitt (1987), and were analyzed more generally in Heckman (1997). Angrist, Graddy, and Imbens (2000) also define and develop a limit form of LATE.
} 
various weights are displayed in Table IB. The other weights, treatment effects and estimands shown in this table are discussed later. A central theme of this paper is that under our assumptions all estimators and estimands can be written as weighted averages of $M T E$.

Observe that if $E\left(\Delta \mid X=x, U_{D}=u_{D}\right)=E(\Delta \mid X=x)$, so $\Delta$ is mean independent of $U_{D}$ given $X=x$, then $\Delta^{M T E}=\Delta^{A T E}=\Delta^{T T}=\Delta^{L A T E}$. Therefore in cases where there is no heterogeneity in terms of unobservables in $\operatorname{MTE}(\Delta$ constant conditional on $X=x)$ or agents do not act on it so that $U_{D}$ drops out of the conditioning set, marginal treatment effects are average treatment effects, so that all of the evaluation parameters are the same. Otherwise, they are different. Only in the case where the marginal treatment effect is the average treatment effect will the "effect" of treatment be uniquely defined.

Figure 1A plots weights for a parametric normal Generalized Roy Model generated from the parameters shown at the base of Figure 1B. We discuss the contents of Figure 1B in section 4. A high $u_{D}$ is associated with higher cost, relative to return, and less likelihood of choosing $D=1$. The decline of $M T E$ in terms of higher values of $u_{D}$ means that people with higher $u_{D}$ have lower gross returns. TT overweights low values of $u_{D}$ (i.e., it oversamples $U_{D}$ that make it likely to have $D=1$ ). ATE samples $U_{D}$ uniformly. Treatment on the Untreated $\left(E\left(Y_{1}-Y_{0} \mid X=x, D=0\right)\right)$ or $T U T$, oversamples the values of $U_{D}$ unlikely to have $D=1$.

Table II shows the treatment parameters produced from the different weighting schemes. Given the decline of the $M T E$ in $u_{D}$, it is not surprising that $T T>A T E>T U T$. The difference between $T T$ and ATE is a sorting gain: $E\left(Y_{1}-Y_{0} \mid X, D=1\right)-E\left(Y_{1}-Y_{0} \mid X\right)$, the average gain experienced by people who sort into treatment compared to what the average person would experience. Purposive selection on the basis of gains should lead to positive sorting gains of the sort found in the table. We return to this table to discuss the other numbers in it.

Heckman (2001) presents evidence on the nonconstancy of the MTE drawn from a variety of studies of schooling, job training, migration and unionism. With the exception of studies of unionism, a common finding in the empirical literature is the nonconstancy of $M T E$ given X. ${ }^{18}$ The evidence from the literature suggests that different treatment parameters measure different effects, and persons participate in programs based on heterogeneity in responses to the program being studied. The phenomenon of nonconstancy of the MTE that we analyze in this paper is of substantial empirical interest.

\footnotetext{
${ }^{18}$ However, most of the empirical evidence is based on parametric models.
} 
The additively separable latent index model for $D$ (equation 3) and assumptions (A-1)-(A-5) are far stronger than what is required to define the parameters in terms of the MTE. The representations of treatment effects defined in Table IA remain valid even if $Z$ is not independent of $U_{D}$, if there are no variables in $Z$ that are not also contained in $X$, or if a more general nonseparable choice model generates $D$ (so $\left.D^{*}=\mu_{D}\left(Z, U_{D}\right)\right)$. No instrument is needed to define the parameters. These issues are discussed further in Section 6.

Assumptions (A-1)-(A-5) will be used to interpret what instrumental variables estimate, and to relate instrumental variables to the policy relevant treatment effects. They are sufficient to identify $\Delta^{M T E}\left(x, u_{D}\right)$ at any $u_{D}$ evaluation point that is a limit point of the support of the distribution of $P(Z)$ conditional on $X=x .{ }^{19}$ As developed in Section 6, without these assumptions and representations (in particular equation (3)) for the choice equations, the $I V$ method and our extension of it does not identify any economically interpretable parameters.

The literature on structural econometrics is clear about the basic parameters of interest although it is not always clear about the exact combinations of parameters needed to answer specific policy problems. $^{20}$ The literature on treatment effects offers a variety of evaluation parameters. Missing from that literature is an algorithm for defining treatment effects that answer precisely formulated policy questions. The MTE provides a framework for developing such an algorithm which we now develop.

\section{Policy Relevant Treatment Parameters}

The conventional treatment parameters do not always answer economically interesting questions. Their link to cost benefit analysis and interpretable economic frameworks is often obscure. ${ }^{21}$ Each answers a different question. Ignoring general equilibrium effects, $\Delta^{T T}$ is one ingredient for determining whether or not a given program should be shut down or retained. It is informative on the question of whether the

\footnotetext{
${ }^{19}$ For example, if we additionally impose that the distribution of $P(Z)$ conditional on $X$ has a density with respect to Lebesgue measure, then (A-1)-(A-5) enable us to identify $\Delta^{M T E}\left(x, u_{D}\right)$ at all $\left(x, u_{D}\right)$ evaluation points in the support of the distribution of $(X, P(Z))$.

${ }^{20}$ In a fundamental paper, Marschak (1953) shows how different combinations of structural parameters are required to forecast the impacts of different policies. It is possible to answer many policy questions without identifying any of the structural parameters individually. The treatment effect literature partially embodies this vision, but typically does not define the economic question being answered, in contrast to Marschak's approach. See Heckman (2001) and Heckman and Vytlacil (2005).

${ }^{21}$ Heckman and Vytlacil (2005) develop the relationship between these parameters and the requirements of cost benefit analysis.
} 
persons participating in a program benefit from it in gross terms. ${ }^{22}$ The parameter $\Delta^{M T E}$ estimates the gross gain from a marginal expansion of a program. Many investigators estimate a treatment effect and hope that it answers an interesting question. A more promising approach to defining parameters is to postulate a policy question or decision problem of interest and to derive the treatment parameter that answers it. Taking this approach does not in general produce the conventional treatment parameters or the estimands produced from instrumental variables.

We consider a class of policies that affect $P$, the probability of participation in a program, but do not affect $\Delta^{M T E}$. The policies analyzed in the treatment effect literature that change the $Z$ not in $X$ are more restrictive than the general policies that shift $X$ and $Z$ analyzed in the structural literature. An example from the schooling literature would be policies that change tuition or distance to school but do not directly affect the gross returns to schooling. Since we ignore general equilibrium effects in this paper, the effects on $\left(Y_{0}, Y_{1}\right)$ from changes in the overall level of education are assumed to be negligible.

Let $a$ and $a^{\prime}$ denote two potential policies and let $D_{a}$ and $D_{a^{\prime}}$ denote the choices that would be made under policies $a$ and $a^{\prime}$. Let the corresponding decision rules be $D_{a}=\mathbf{1}\left[P_{a}\left(Z_{a}\right) \geq U_{D}\right], D_{a^{\prime}}=\mathbf{1}\left[P_{a^{\prime}}\left(Z_{a^{\prime}}\right) \geq\right.$ $\left.U_{D}\right]$, where $P_{a}\left(Z_{a}\right)=\operatorname{Pr}\left(D_{a}=1 \mid Z_{a}\right)$ and $P_{a^{\prime}}\left(Z_{a^{\prime}}\right)=\operatorname{Pr}\left(D_{a^{\prime}}=1 \mid Z_{a^{\prime}}\right)$. To simplify the exposition, we will suppress the arguments of these functions and write $P_{a}$ and $P_{a^{\prime}}$ for $P_{a}\left(Z_{a}\right)$ and $P_{a^{\prime}}\left(Z_{a^{\prime}}\right)$. Define $\left(Y_{0, a}, Y_{1, a}, U_{D, a}\right)$ as $\left(Y_{0}, Y_{1}, U_{D}\right)$ under policy $a$, and define $\left(Y_{0, a^{\prime}}, Y_{1, a^{\prime}}, U_{D, a^{\prime}}\right)$ correspondingly under policy $a^{\prime}$. We assume that $Z_{a}$ and $Z_{a^{\prime}}$ are independent respectively of $\left(Y_{0, a}, Y_{1, a}, U_{D, a}\right)$ and $\left(Y_{0, a^{\prime}}, Y_{1, a^{\prime}}, U_{D, a^{\prime}}\right)$ conditional on $X_{a}$ and $X_{a^{\prime}}$. Let $Y_{a}=D_{a} Y_{1, a}+\left(1-D_{a}\right) Y_{0, a}$ and $Y_{a^{\prime}}=D_{a^{\prime}} Y_{1, a^{\prime}}+\left(1-D_{a^{\prime}}\right) Y_{0, a^{\prime}}$, denote the outcomes that would be observed under policies $a$ and $a^{\prime}$, respectively.

We define $\Delta^{M T E}$ as policy invariant if

Policy Invariance $E\left(Y_{1, a} \mid U_{D, a}=u, X_{a}=x\right)$ and $E\left(Y_{0, a} \mid U_{D, a}=u, X_{a}=x\right)$ are invariant to the choice of policy $a$.

Policy invariance can be justified by the strong assumption that the policy change does not change the counterfactual outcomes, covariates, or unobservables, i.e., $\left(Y_{0, a}, Y_{1, a}, X_{a}, U_{D, a}\right)=\left(Y_{0, a^{\prime}}, Y_{1, a^{\prime}}, X_{a^{\prime}}, U_{D, a^{\prime}}\right)$. However, $\Delta^{M T E}$ is policy invariant if this assumption is relaxed to the weaker assumption that the policy change does not affect the distribution of these variables conditional on $X$ :

\footnotetext{
${ }^{22}$ It is necessary to account for costs to conduct a proper cost benefit analysis. See the discussion in Heckman and Vytlacil (2005) for nonparametric cost-benefit analysis.
} 
(A-7) The distribution of $\left(Y_{0, a}, Y_{1, a}, U_{D, a}\right)$ conditional on $X_{a}=x$ is the same as the distribution of $\left(Y_{0, a^{\prime}}, Y_{1, a^{\prime}}, U_{D, a^{\prime}}\right)$ conditional on $X_{a^{\prime}}=x$.

We assume (A-7) holds and discuss invariance further in Appendix B.

For the widely used Benthamite social welfare criterion $V(Y)$, comparing policies using mean outcomes, and considering the effect for individuals with a given level of $X=x$, we obtain the policy relevant treatment effect, PRTE, denoted $\Delta^{P R T E}(x)$ :

$$
E\left(V\left(Y_{a}\right) \mid X=x\right)-E\left(V\left(Y_{a^{\prime}}\right) \mid X=x\right)=\int_{0}^{1} \Delta_{V}^{M T E}\left(x, u_{D}\right)\left\{F_{P_{a^{\prime}} \mid X}\left(u_{D} \mid x\right)-F_{P_{a} \mid X}\left(u_{D} \mid x\right)\right\} d u_{D}
$$

where $F_{P_{a} \mid X}(\cdot \mid x)$ and $F_{P_{a^{\prime}} \mid X}(\cdot \mid x)$ are the distributions of $P_{a}$ and $P_{a^{\prime}}$ conditional on $X=x$, respectively, defined for the different policy regimes and $\Delta_{V}^{M T E}=E\left(V\left(Y_{1, a}\right)-V\left(Y_{0, a}\right) \mid U_{D, a}=u, X_{a}=x\right){ }^{23,24}$ The weights are derived in Appendix B under the assumption that the policy does not change the joint distribution of outcomes. To simplify the notation, throughout the rest of this paper, we assume that $V(Y)=Y$. Modifications of our analysis for the more general case are straightforward.

Define $\Delta \bar{P}(x)=E\left(P_{a} \mid X=x\right)-E\left(P_{a^{\prime}} \mid X=x\right)$, the change in the proportion of people induced into the program due to the intervention. Assuming $\Delta \bar{P}(x)$ is positive, we may define per person affected weights as $h_{P R T E}\left(x, u_{D}\right)=\frac{F_{P_{a^{\prime}} \mid X}\left(u_{D} \mid x\right)-F_{P_{a} \mid X}\left(u_{D} \mid x\right)}{\Delta \bar{P}(x)}$. These are the weights displayed in Table IB. As demonstrated in the next section, in general, conventional $I V$ weights $\Delta_{V}^{M T E}$ differently than either the conventional treatment parameters $\left(\Delta^{A T E}\right.$ or $\left.\Delta^{T T}\right)$ or the policy relevant parameters, and so does not recover these parameters.

Instead of hoping that conventional treatment parameters or favorite estimators answer interesting economic questions, one approach developed in this paper is to estimate $\Delta_{V}^{M T E}$ and weight it by the appropriate weight determined by how the policy changes the distribution of $P$ to construct $\Delta^{P R T E}$. An alternative approach produces a policy weighted instrument to identify $\Delta^{P R T E}$ by standard instrumental

\footnotetext{
${ }^{23}$ We could define policy invariance for $\Delta^{M T E}$ in terms of expectations of $V\left(Y_{1, a}\right)$ and $V\left(Y_{0, a}\right)$.

${ }^{24}$ If we assume that the marginal distribution of $X_{a}$ and $X_{a^{\prime}}$ are the same as the marginal distribution of a benchmark $X$, the weights can be integrated against the distribution of $X$ to obtain the total effect of the policy in the population:

$$
\begin{aligned}
E\left(V\left(Y_{a}\right)\right)-E\left(V\left(Y_{a^{\prime}}\right)\right)=E_{X}\left\{E\left(V\left(Y_{a}\right) \mid X\right)-E\right. & \left.\left(V\left(Y_{a^{\prime}}\right) \mid X\right)\right\} \\
& =\int\left[\int_{0}^{1} \Delta_{V}^{M T E}\left(x, u_{D}\right)\left\{F_{P_{a^{\prime}} \mid X}\left(u_{D} \mid x\right)-F_{P_{a} \mid X}\left(u_{D} \mid x\right)\right\} d u_{D}\right] d F_{X}(x) .
\end{aligned}
$$
}


variables. We develop both approaches in the next section. Before doing so, we first consider what conventional $I V$ estimates and conditions for identifying $\Delta^{M T E}$. We also consider matching methods and $O L S$.

\section{Instrumental Variables, Local Instrumental Variables, $O L S$ and Matching}

In this section, we use $\Delta^{M T E}$ to organize the literature on econometric evaluation estimators. We assume (A-7) but for simplicity suppress the " $a$ " and " $a$ "' subscripts. We focus primarily on instrumental variable estimators but also briefly consider the method of matching. We present the method of local instrumental variables. Well established intuitions about instrumental variable identification strategies break down when $\Delta^{M T E}$ is nonconstant in $u_{D}$ given $X$. Two sets of instrumental variable conditions are presented in the current literature for this more general case: those associated with conventional instrumental variable assumptions which are implied by the assumption of "no selection on heterogenous gains" and those which permit selection on heterogeneous gains. Neither set implies the other, nor does either identify the policy relevant treatment effect in the general case. Each set of conditions identifies different treatment parameters.

In place of standard instrumental variables methods, we advocate a new approach to estimating policy impacts by estimating $\Delta^{M T E}$ using local instrumental variables $(L I V)$ to identify all of the treatment parameters from a generator $\Delta^{M T E} . \Delta^{M T E}$ can be weighted in different ways to answer different policy

questions. For certain classes of policy interventions discussed in Section $5, \Delta^{M T E}$ possesses an invariance property analogous to the invariant parameters of traditional structural econometrics.

We also consider whether it is possible to construct an instrument such that instrumental variables directly estimate $\Delta^{P R T E}$. We establish necessary and sufficient conditions for the existence of such an instrument. We also address the inverse question of whether instrumental variable estimators always answer well-posed policy questions. In general, they do not. We present necessary and sufficient conditions for a particular instrument to answer some policy counterfactual and characterize what question is answered when an answer exists. 


\subsection{Conventional Instrumental Variables}

In the general case with $\Delta^{M T E}\left(x, u_{D}\right)$ nonconstant in $u_{D}$, linear $I V$ does not estimate any of the treatment effects previously defined. Let $J(Z)$ denote an instrument written as a function of $Z$. We sometimes denote $J(Z)$ by $J$, leaving implicit that $J$ is a function of $Z$. The standard conditions $J(Z) \not \Perp \Perp ~\left(U_{1}, U_{0}\right)$ and $\operatorname{Cov}(J(Z), D) \neq 0$ do not, by themselves, imply that instrumental variables using $J(Z)$ as the instrument will identify conventional or policy-relevant treatment effects. We must supplement the standard conditions to identify interpretable parameters. To link our analysis to conventional analyses of $I V$, we invoke familiarlooking representations of additive separability of outcomes in terms of $\left(U_{1}, U_{0}\right)$ so $Y_{1}=\mu_{1}(X)+U_{1}$ and $Y_{0}=\mu_{0}(X)+U_{0}$, but this is not strictly required. All derivations and results in this section hold without any additive separability assumption if $\mu_{1}(x)$ and $\mu_{0}(x)$ are replaced by $E\left(Y_{1} \mid X=x\right)$ and $E\left(Y_{0} \mid X=x\right)$, respectively, and $U_{1}$ and $U_{0}$ are replaced by $Y_{1}-E\left(Y_{1} \mid X\right)$ and $Y_{0}-E\left(Y_{0} \mid X\right)$, respectively.

Two distinct sets of instrumental variable conditions in the literature are those due to Heckman and Robb (1985, 1986) and Heckman (1997), and those due to Imbens and Angrist (1994). In the case where $\Delta^{M T E}$ is nonconstant in $u_{D}$, linear $I V$ estimates different parameters depending on which assumptions are maintained. To establish this point, it is useful to briefly review the $I V$ method in the case of a common treatment effect defined conditional on $X$, where $Y_{1}-Y_{0}=\Delta$, with $\Delta$ a deterministic function of $X$, and where additive separability in outcomes is assumed, as in conventional models. Using (1a) and (1b) with $U_{1}=U_{0}=U$, and assuming $E(U \mid X)=0$, we may write $Y=\mu_{0}(X)+D \Delta+U$ where $\Delta=\mu_{1}(X)-\mu_{0}(X)$. By the law of iterated expectations, $E(U \mid X)=0$ and $Z \Perp U \mid X$ imply $E(U J(Z) \mid X)=0$. The standard instrumental variables intuition is that when $E(U J \mid X)=0$ and $\operatorname{Cov}(J, D \mid X) \neq 0$, linear $I V$ identifies $\Delta$ :

$(I V) \frac{\operatorname{Cov}(J, Y \mid X)}{\operatorname{Cov}(J, D \mid X)}=\frac{\operatorname{Cov}(J, D \Delta \mid X)}{\operatorname{Cov}(J, D \mid X)}=\Delta \frac{\operatorname{Cov}(J, D \mid X)}{\operatorname{Cov}(J, D \mid X)}=\Delta=\mu_{1}(X)-\mu_{0}(X)$

where the second equality follows from the assumption that $\Delta$ is a deterministic function of $X$. This intuition breaks down in the heterogeneous response case where the outcomes are generated by different unobservables $\left(U_{0} \neq U_{1}\right)$ so $Y=\mu_{0}(X)+D \Delta+U_{0}$, where $\Delta=\mu_{1}(X)-\mu_{0}(X)+U_{1}-U_{0}$. This is a variable response model.

There are two important cases of the variable response model. The first case arises when responses are heterogeneous, but conditional on $X$ : people do not base their participation on these responses. In this 
case, the following condition holds:

(C-1) $D \Perp \Delta \mid X \Longrightarrow E\left(\Delta \mid X, U_{D}\right)=E(\Delta \mid X), \Delta^{M T E}\left(x, u_{D}\right)$ is constant in $u_{D}$ and $\Delta^{M T E}=\Delta^{A T E}=$ $\Delta^{T T}=\Delta^{L A T E}$.

The second case arises when the following condition holds:

(C-2) $D \not \Perp \Delta \mid X$ and $E\left(\Delta \mid X, U_{D}\right) \neq E(\Delta \mid X)$.

In this case $\Delta^{M T E}$ is nonconstant and the treatment parameters differ among each other.

Application of the standard $I V$ equation to the general variable coefficient model produces the first equality in $I V$ above. Now, however, $\Delta$ is not a deterministic function of $X$ and thus we cannot simply take $\Delta$ outside of the covariance term as in the third term of $(I V)$. Plugging in $\Delta=\mu_{1}(X)-\mu_{0}(X)+U_{1}-U_{0}$, we obtain

$$
\frac{\operatorname{Cov}(J, D \Delta \mid X)}{\operatorname{Cov}(J, D \mid X)}=\mu_{1}(X)-\mu_{0}(X)+\frac{\operatorname{Cov}\left(J, D\left(U_{1}-U_{0}\right) \mid X\right)}{\operatorname{Cov}(J, D \mid X)} .
$$

Our independence assumptions imply that $J$ is independent of $U_{1}-U_{0}$ conditional on $X$, but do not imply that $J$ is uncorrelated with $D\left(U_{1}-U_{0}\right)$ conditional on $X$. Thus, in general, the covariance in the numerator of the second term is not zero. Knowledge of $(X, Z, D)$ and $\left(X, Z,\left(U_{0}, U_{1}\right)\right)$ dependencies is not enough to determine the covariance in the second term. We need to know joint $\left(X, Z, D, U_{0}, U_{1}\right)$ dependencies.

A sufficient condition for producing $(\mathrm{C}-1)$ is the strong information condition that decisions to participate in the program are not made on the basis of $U_{1}-U_{0}$ :

$$
\operatorname{Pr}\left(D=1 \mid Z, X, U_{1}-U_{0}\right)=\operatorname{Pr}(D=1 \mid Z, X)
$$

Given our assumption that $\left(U_{1}-U_{0}\right)$ is independent of $Z$ given $X$, one can use Bayes' Theorem to show that (I-1) implies the weaker mean independence condition:

$$
E\left(U_{1}-U_{0} \mid Z, X, D=1\right)=E\left(U_{1}-U_{0} \mid X, D=1\right)
$$

which is generically necessary and sufficient for linear $I V$ to identify $\Delta^{T T}$ and $\Delta^{A T E}$.

Case (C-2) is inconsistent with (I-2). IV estimates $\Delta^{L A T E}$ under the conditions of Imbens and Angrist (1994). $\Delta^{L A T E}$, selection models, and $L I V$, introduced below, analyze the more general case covered by 
(C-2). Different assumptions define different parameters. In addition, as we establish in Section 4.3, even under the same assumptions, different instruments define different parameters and traditional intuitions about instrumental variables break down.

\subsection{Estimating the $M T E$ Using Local Instrumental Variables}

Heckman and Vytlacil $(1999,2001 b)$ resolve this confusion using the Local Instrumental Variable $(L I V)$ estimator to recover $\Delta^{M T E}$ pointwise. Conditional on $X=x, L I V$ is the derivative of the conditional expectation of $Y$ with respect to $P(Z)=p$ :

$$
\Delta^{L I V}(x, p) \equiv \frac{\partial E(Y \mid X=x, P(Z)=p)}{\partial p}
$$

$E\left(Y_{1}-Y_{0} \mid X, P(Z)\right)$ exists (almost everywhere) by assumption (A-4), and $E(Y \mid X, P(Z))$ can be recovered over the support of $(X, P(Z))$. Assumptions (A-2), (A-3) and (A-4) jointly allow one to use Lebesgue's theorem for the derivative of an integral to show that $E\left(Y_{1}-Y_{0} \mid X=x, P(Z)=p\right)$ is differentiable in $p$. Thus we can recover $\frac{\partial}{\partial p} E(Y \mid X=x, P(Z)=p)$ for almost all $p$ that are limit points of the support of distribution of $P(Z)$ conditional on $X=x \cdot{ }^{25}$ Under our assumptions, $L I V$ identifies $M T E$ for all limit points in the support of the distribution of $P(Z)$ conditional on $X$. This expression does not require additive separability of $\mu_{1}\left(X, U_{1}\right)$ or $\mu_{0}\left(X, U_{0}\right) \cdot{ }^{26}$

Under standard regularity conditions, a variety of nonparametric methods can be used to estimate the derivative of $E(Y \mid X, P(Z))$ and thus to estimate $\Delta^{M T E}$. With $\Delta^{M T E}$ in hand, if the support of the distribution of $P(Z)$ conditional on $X$ is the full unit interval, one can generate all the treatment parameters defined in Section 2 as well as the policy relevant treatment parameter presented in Section 3 as weighted versions of $\Delta^{M T E}$. When the support of the distribution of $P(Z)$ conditional on $X$ is not full, it is still possible to identify some parameters. For example, Heckman and Vytlacil (2001b) show that to identify $A T E$ under our assumptions, it is necessary and sufficient that the support of the distribution of $P(Z)$

\footnotetext{
${ }^{25}$ For example, if the distribution of $P(Z)$ conditional on $X$ has a density with respect to Lebesgue measure, then all points in the support of the distribution of $P(Z)$ conditional on $X$ are limit points of that support and we can identify $\Delta^{L I V}(x, p)=\frac{\partial E(Y \mid X=x, P(Z)=p)}{\partial p}$ for $p$ (almost everywhere).

${ }^{26}$ Note, however, it does require our model and assumptions, including the assumption of additive separability between $U_{D}$ and $Z$ in the latent index for selection into treatment. See the discussion in Section 6.
} 
conditional on $X$ includes 0 and 1. Thus, identification of ATE does not require that the distribution of $P(Z)$ conditional on $X$ be the full unit interval or that the distribution of $P(Z)$ conditional on $X$ contain any limit points. Sharp bounds on the treatment parameters can be constructed under the same assumptions imposed in this paper without imposing full support conditions. The resulting bounds are simple and easy to apply compared with those presented in the previous literature. ${ }^{27}$

To establish the relationship between $L I V$ and ordinary $I V$ based on $P(Z)$ and to motivate how $L I V$ identifies $\Delta^{M T E}$, notice that from the definition of $Y$ that the conditional expectation of $Y$ given $P(Z)$ is

$$
E(Y \mid P(Z)=p)=E\left(Y_{0} \mid P(Z)=p\right)+E(\Delta \mid P(Z)=p, D=1) p,
$$

where we keep the conditioning on $X$ implicit. Our model and conditional independence assumption (A-2) imply

$$
E(Y \mid P(Z)=p)=E\left(Y_{0}\right)+E\left(\Delta \mid p \geq U_{D}\right) p
$$

Applying the $I V$ or Wald estimator for two different values of $P(Z), p$ and $p^{\prime}$, for $p \neq p^{\prime}$, we obtain:

$$
\frac{E(Y \mid P(Z)=p)-E\left(Y \mid P(Z)=p^{\prime}\right)}{p-p^{\prime}}=\Delta^{A T E}+\frac{E\left(U_{1}-U_{0} \mid p \geq U_{D}\right) p-E\left(U_{1}-U_{0} \mid p^{\prime} \geq U_{D}\right) p^{\prime}}{p-p^{\prime}}
$$

where the expression is obtained under the assumption of additive separability in the outcomes so (1a) and (1b) apply. Note that exactly the same equation holds without additive separability if one replaces $U_{1}$ and $U_{0}$ with $Y_{1}-E\left(Y_{1} \mid X\right)$ and $Y_{0}-E\left(Y_{0} \mid X\right)$.

When $U_{1} \equiv U_{0}$ or $\left(U_{1}-U_{0}\right) \Perp U_{D}$, (case $\left.(\mathrm{C}-1)\right), I V$ based on $P(Z)$ estimates $\Delta^{A T E}$ because the second term on the right hand side of the expression (8) vanishes. Otherwise, $I V$ estimates a difficult-to-interpret combination of MTE parameters which we analyze further below.

Another representation of $E(Y \mid P(Z)=p)$ that reveals the index structure under additive separability more explicitly writes (keeping the conditioning on $X$ implicit) that

$$
E(Y \mid P(Z)=p)=E\left(Y_{0}\right)+\Delta^{A T E} p+\int_{0}^{p} E\left(U_{1}-U_{0} \mid U_{D}=u_{D}\right) d u_{D}
$$

\footnotetext{
${ }^{27}$ For example, see Heckman and Vytlacil (2001a) for a comparison of sharp bounds under the nonparametric selection model with the Manski (1990) sharp bounds under a weaker mean independence condition. Heckman and Vytlacil (2005) survey and synthesize this literature and Heckman and Vytlacil (2001b) develop the bounds.
} 
We can differentiate with respect to $p$ and use $L I V$ to identify $\Delta^{M T E}$ :

$$
\frac{\partial E(Y \mid P(Z)=p)}{\partial p}=\Delta^{A T E}+E\left(U_{1}-U_{0} \mid U_{D}=p\right)=\Delta^{M T E}(p)
$$

Notice that $I V$ estimates $\Delta^{A T E}$ when $E(Y \mid P(Z)=p)$ is a linear function of $p$. Thus a test of the linearity of $E(Y \mid P(Z)=p)$ in $p$ is a test of the validity of linear $I V$ for $\Delta^{A T E}$, i.e., it is a test of whether or not the data are consistent with a correlated random coefficient model. The nonlinearity of $E(Y \mid P(Z)=p)$ in $p$ provides a way to distinguish whether Case $(\mathrm{C}-1)$ or Case (C-2) describes the data. It is also a test of whether or not agents can at least partially anticipate future unobserved (by the econometrician) gains (the $Y_{1}-Y_{0}$ given $X$ ) at the time they make their participation decisions. This analysis generalizes to the nonseparable outcomes case. We use separability in outcomes only to simplify the exposition and link to more traditional models. In particular, exactly the same expression holds with exactly the same derivation for the nonseparable case if we replace $U_{1}$ and $U_{0}$ with $Y_{1}-E\left(Y_{1} \mid X\right)$ and $Y_{0}-E\left(Y_{0} \mid X\right)$, respectively. ${ }^{28}$

Figure 2A plots two cases of $E(Y \mid P(Z)=p)$ based on the Generalized Roy Model used to generate the example in Figure $1 \mathrm{~A}$ and $1 \mathrm{~B}$. When $\Delta^{M T E}$ does not depend on $u_{D}$ the expectation is a straight line. Figure $2 \mathrm{~B}$ plots the derivatives of the two curves in Figure $2 \mathrm{~A}$. When $\Delta^{M T E}$ depends on $u_{D}$, people sort into the program being studied positively on the basis of gains from the program, and one gets the curved line depicted in Figure 2A. The levels and derivatives of $E(Y \mid P(Z)=p)$ and standard errors can be estimated using a variety of semiparametric methods. The derivative estimator of $\Delta^{M T E}$ is the local instrumental variable $(L I V)$ estimator of Heckman and Vytlacil (1999, 2001b). Thus it is possible to test condition (C-1) using simple econometric methods. In the case without regressors, $X$, the null hypothesis is the parametric null of linearity. ${ }^{29}$

\footnotetext{
${ }^{28}$ Making the conditioning on $X$ explicit, we obtain that $E(Y \mid X=x, P(Z)=p)=E\left(Y_{0} \mid X=x\right)+\Delta^{A T E}(x) p+$ $\int_{0}^{p} E\left(U_{1}-U_{0} \mid X=x, U_{d}=u_{D}\right) d u_{D}$, with derivative with respect to $p$ given by $\Delta^{M T E}(x, p)$.

${ }^{29}$ Thus, one can apply any one of the large number of available tests for a parametric null versus a nonparametric alternative (see, e.g., Ellison and Ellison, 1999; Zheng, 1996). With regressors, the null is nonparametric leaving $E(Y \mid X=x, P(Z)=p)$ unspecified except for restrictions on the partial derivatives with respect to $p$. In this case, the formal test is a test of a nonparametric null versus a nonparametric alternative, and a formal test of the null hypothesis can be implemented using the methodology of Chen and Fan (1999).
} 


\subsection{What Does Linear IV Estimate?}

It is instructive to consider what linear $I V$ estimates when $\Delta^{M T E}$ is nonconstant, and conditions (A-1)(A-5) hold. We consider the general nonseparable case. We consider instrumental variables conditional on $X=x$ using a general function of $Z$ as an instrument, and then specialize our result using $P(Z)$ as the instrument. Let $J(Z)$ be any function of $Z$ such that $\operatorname{Cov}(J(Z), D \mid X=x) \neq 0$. Define

$$
\beta_{I V}(x ; J) \equiv[\operatorname{Cov}(J(Z), Y \mid X=x)] /[\operatorname{Cov}(J(Z), D \mid X=x)]
$$

Appendix B derives an expression for the numerator of this expression, using (1c) and (A-2) and letting $\tilde{J}(Z) \equiv J(Z)-E(J(Z) \mid X)$

$$
\operatorname{Cov}(J(Z), Y \mid X)=\int_{0}^{1} \Delta^{M T E}\left(X, u_{D}\right) E\left(\tilde{J}(Z) \mid X, P(Z) \geq u_{D}\right) \operatorname{Pr}\left(P(Z) \geq u_{D} \mid X\right) d u_{D}
$$

The denominator follows by a similar argument. By iterated expectations $\operatorname{Cov}(J(Z), D \mid X)=\operatorname{Cov}(J(Z), P(Z) \mid$ Thus

$$
\beta_{I V}(x ; J)=\int \Delta^{M T E}\left(x, u_{D}\right) h_{I V}\left(u_{D} \mid x ; J\right) d u_{D}
$$

where

$$
h_{I V}\left(u_{D} \mid x ; J\right)=\frac{E\left(\tilde{J}(Z) \mid X=x, P(Z) \geq u_{D}\right) \operatorname{Pr}\left(P(Z) \geq u_{D} \mid X=x\right)}{\operatorname{Cov}(J(Z), P(Z) \mid X=x)},
$$

assuming the standard rank condition $\operatorname{Cov}(J(Z), P(Z) \mid X=x) \neq 0$. The weights integrate to unity,

$$
\int_{0}^{1} h_{I V}\left(u_{D} \mid x ; J\right) d u_{D}=1
$$

and can be constructed from the data on $X, P(Z), J(Z)$ and $D$. Assumptions about the properties of the weights are testable. ${ }^{30}$

We first discuss additional properties of the weights for the special case where $J(Z)=P(Z)$ (the propensity score is the instrument), and then analyze the properties of the weights for a general instrument

\footnotetext{
${ }^{30}$ Expressions for $I V$ and $O L S$ as weighted averages of marginal response functions, and the properties and construction of the weights, were first derived by Yitzhaki in 1989 in a paper that was eventually published in 1996 (See Yitzhaki, 1996). He does not use the MTE, however.
} 
$J(Z)$. From equation (11),

$$
h_{I V}\left(u_{D} \mid x ; P(Z)\right)=\frac{\left[E\left(P(Z) \mid X=x, P(Z) \geq u_{D}\right)-E(P(Z) \mid X=x)\right] \operatorname{Pr}\left(P(Z) \geq u_{D} \mid X=x\right)}{\operatorname{Var}(P(Z) \mid X=x)} .
$$

Figure 1B plots the $I V$ weight for $J(Z)=P(Z)$ and the $M T E$ for our Generalized Roy model example (see also Table $1 \mathrm{~B})$. Let $p_{x}^{\mathrm{Min}}$ and $p_{x}^{\mathrm{Max}}$ denote the minimum and maximum points in the support of the distribution of $P(Z)$ conditional on $X=x$. The weights on $M T E$ corresponding to the use of $P(Z)$ as the instrument are nonnegative for all evaluation points, are strictly positive for $u_{D} \in\left(p_{x}^{\mathrm{Min}}, p_{x}^{\mathrm{Max}}\right)$ and are zero for $u_{D}<p_{x}^{\operatorname{Min}}$ and for $u_{D}>p_{x}^{\operatorname{Max}} \cdot 31$

Our expression for the weights does not impose any support conditions on the distribution of $P(Z)$ conditional on $X$, and thus does not require either that $P(Z)$ be continuous or discrete. To demonstrate this, consider two extreme special cases: (i) when $P(Z)$ is a continuous random variable, and (ii) when $P(Z)$ is a discrete random variable.

First consider the case where the distribution of $P(Z)$ conditional on $X$ has a density with respect to Lebesgue measure with nonnegative density on the interval ( $\left.p_{x}^{\mathrm{Min}}, p_{x}^{\mathrm{Max}}\right)$. In this case, $\Delta^{L I V}\left(x, u_{D}\right)$ is well defined for all $u_{D} \in\left(p_{x}^{\mathrm{Min}}, p_{x}^{\mathrm{Max}}\right)$ such that $h_{I V}\left(u_{D} \mid x ; P(Z)\right)>0$. Using the fact that $\Delta^{L I V}\left(x, u_{D}\right)=$ $\Delta^{M T E}\left(x, u_{D}\right)$ at evaluation points where $L I V$ is well defined, we can rewrite the expression for the $I V$ estimator as

$$
\beta_{I V}(x ; P(Z))=\int_{p_{x}^{\mathrm{Min}}}^{p_{x}^{\mathrm{Max}}} \Delta^{L I V}\left(x, u_{D}\right) h_{I V}\left(u_{D} \mid x ; P(Z)\right) d u_{D} \cdot{ }^{32}
$$

Next consider the case where the distribution of $P(Z)$ conditional on $X$ has density with respect to counting measure. For simplicity, assume that the support of the distribution of $P(Z)$ conditional on $X$

${ }^{31}$ For $u_{D}$ evaluation points between $p_{x}^{\mathrm{Min}}$ and $p_{x}^{\mathrm{Max}}, u_{D} \in\left(p_{x}^{\mathrm{Min}}, p_{x}^{\mathrm{Max}}\right)$, we have that

$$
E\left(P(Z) \mid P(Z) \geq u_{D}, X=x\right)>E(P(Z) \mid X=x) \text { and } \operatorname{Pr}\left(P(Z) \geq u_{D} \mid X=x\right)>0,
$$

so that $h_{I V}\left(u_{D} \mid x ; P(Z)\right)>0$ for any $u_{D} \in\left(p_{x}^{\mathrm{Min}}, p_{x}^{\mathrm{Max}}\right)$. For $u_{D}<p_{x}^{\mathrm{Min}}$,

$$
E\left(P(Z) \mid P(Z) \geq u_{D}, X=x\right)=E(P(Z) \mid X=x) .
$$

For any $u_{D}>p_{x}^{\mathrm{Max}}, \operatorname{Pr}\left(P(Z) \geq u_{D} \mid X=x\right)=0$. Thus, $h_{I V}\left(u_{D} \mid x ; P(Z)\right)=0$ for any $u_{D}<p_{x}^{\mathrm{Min}}$ and for any $u_{D}>p_{x}^{\mathrm{Max}}$. $h_{I V}\left(u_{D} \mid x ; P(Z)\right)$ is strictly positive for $u_{D} \in\left(p_{x}^{\mathrm{Min}}, p_{x}^{\mathrm{Max}}\right)$, and is zero for all $u_{D}<p_{x}^{\text {Min }}$ and all $u_{D}>p_{x}^{\mathrm{Max}}$. Whether the weights are nonzero at the endpoints depends on the distribution of $P(Z)$. However, since the weights are defined for integration with respect to Lebesgue measure, the value taken by the weights at $p_{x}^{\text {Min }}$ and $p_{x}^{\text {Max }}$ does not affect the value of the integral.

${ }^{32}$ Angrist, Graddy, and Imbens (2000) develop a special case of this expression for a scalar instrument. 
contains a finite number of values, $\left\{p_{1}, \ldots, p_{K}\right\}$ with $p_{1}<p_{2}<\cdots<p_{K}$. Then $E(P(Z) \mid X=x, P(Z) \geq$ $\left.u_{D}\right)$ is constant in $u_{D}$ for $u_{D}$ within any $\left(p_{j}, p_{j+1}\right)$ interval, and $\operatorname{Pr}\left(P(Z) \geq u_{D}\right)$ is constant in $u_{D}$ for $u_{D}$ within any $\left(p_{j}, p_{j+1}\right)$ interval, and thus $h_{I V}\left(u_{D} \mid x ; P(Z)\right)$ is constant in $u_{D}$ over any $\left(p_{j}, p_{j+1}\right)$ interval. Let $q_{j}$ denote the value taken by $h_{I V}\left(u_{D} \mid x ; P(Z)\right)$ for $u_{D} \in\left(p_{j}, p_{j}+1\right)$. Then, letting $\tilde{q}_{j}=q_{j}\left(p_{j+1}-p_{j}\right)$

$$
\begin{aligned}
\beta_{I V}(x ; P(Z)) & =\int E\left(\Delta \mid X=x, U_{D}=u_{D}\right) h_{I V}\left(u_{D} \mid x ; P(Z)\right) d u_{D} \\
& =\sum_{j=1}^{K-1} \int_{p_{j}}^{p_{j+1}} E\left(\Delta \mid X=x, U_{D}=u_{D}\right) q_{j} d u_{D} \\
& =\sum_{j=1}^{K-1} q_{j}\left(p_{j+1}-p_{j}\right) \int_{p_{j}}^{p_{j+1}} E\left(\Delta \mid X=x, U_{D}=u_{D}\right) \frac{1}{\left(p_{j+1}-p_{j}\right)} d u_{D} \\
& =\sum_{j=1}^{K-1} \Delta^{L A T E}\left(x, p_{j}, p_{j+1}\right) \tilde{q}_{j} .{ }^{33}
\end{aligned}
$$

The properties of the weights for general $J(Z)$ depend critically on the relationship between $J(Z)$ and $P(Z)$. Defining $T(p \mid x ; J)=E(J \mid P(Z)=p, X=x)-E(J \mid X=x)$,

$$
h_{I V}\left(u_{D} \mid x ; J\right)=\frac{\int_{u_{D}}^{1} T(t \mid x ; J) d F_{P \mid X}(t \mid x)}{\operatorname{Cov}(J, P \mid X=x)} .
$$

From this expression, we learn that the $I V$ estimator with $J(Z)$ as an instrument satisfies the following properties:

(i) Two instruments $J$ and $J^{*}$ weight $M T E$ equally at all $u_{D}$ evaluation points if and only if $E(J \mid X=$ $x, P(Z)=p)-E(J \mid X=x)=E\left(J^{*} \mid X=x, P(Z)=p\right)-E\left(J^{*} \mid X=x\right)$ for all $p$ in the support of the distribution of $P(Z)$ conditional on $X=x$.

(ii) The support of $h_{I V}\left(u_{D} \mid x ; J\right)$ is contained in $\left(p_{x}^{\operatorname{Min}}, p_{x}^{\operatorname{Max}}\right)$. Therefore $h_{I V}(t \mid x ; J)=0$ for $t<p_{x}^{\text {Min }}$ and for $t>p_{x}^{\text {Max }}$. Using any instrument other than $P(Z)$ leads to nonzero weights only on a subset of $\left(p_{x}^{\operatorname{Min}}, p_{x}^{\mathrm{Max}}\right)$, and using the propensity score as an instrument leads to nonnegative weights on a larger range of evaluation points than using any other instrument.

(iii) $h_{I V}\left(u_{D} \mid x ; J\right)$ is nonnegative for all $u_{D}$ if $E(J \mid X=x, P(Z) \geq p)$ is weakly monotonic in $p$. Using $J$

\footnotetext{
${ }^{33}$ In this special case, our analysis is a latent variable version of the formula in Imbens and Angrist (1994).
} 
as an instrument yields nonnegative weights on $\Delta^{M T E}$ if $E(J \mid X=x, P(Z) \geq p)$ is weakly monotonic in $p$. This condition is satisfied when $J(Z)=P(Z)$. More generally, if $J$ is a monotonic function of $P(Z)$, then using $J$ as the instrument will lead to nonnegative weights on $\Delta^{M T E}$. There is no guarantee that the weights for a general $J(Z)$ will be nonnegative for all $u_{D}$, although the weights integrate to unity and thus must be positive over some range of evaluation points. We produce examples below where the instrument leads to negative weights for some evaluation points.

The propensity score plays a central role in determining the properties of the weights. The $I V$ weighting formula critically depends on $T(p \mid x ; J)$ and hence on the relationship between the instrument $J(Z)$ and the propensity score. For example, whether two instruments provide the same weights on MTE depends on their relationship with $P(Z)$ (item (i) above), the possible support of the $I V$ weights depends on the support of $P(Z)$ (item (ii)) and whether an instrument will provide positive weights on $M T E$ depends on the instrument's relationship with $P(Z)$ (item (iii)).

The interpretation placed on the $I V$ estimand depends on the specification of $P(Z)$ even if only $Z_{1}$ (e.g. a coordinate of $Z$ ) is used as the instrument. This drives home the point about the difference between $I V$ in the traditional model and $I V$ in the more general model with heterogeneous responses analyzed in this paper. In the traditional model, the choice of any valid instrument and the specification of instruments in $P(Z)$ not used to construct a particular $I V$ estimator does not affect the $I V$ estimand. In the more general model analyzed in this paper, these choices matter. Two economists, using the same $J(Z)=Z_{1}$, will obtain the same $I V$ point estimate, but the interpretation placed on that estimate will depend on the specification of the $Z$ in $P(Z)$ even if $P(Z)$ is not used as an instrument. The weights can be positive for one instrument and negative for another.

Table II gives the $I V$ estimand for the Generalized Roy Model used to generate Figures $1 \mathrm{~A}$ and $1 \mathrm{~B}$ using $P(Z)$ as the instrument. The model generating $D=\mathbf{1}\left[\beta^{\prime} Z>V\right]$ is given at the base of Figure $1 \mathrm{~B}$ ( $Z$ is a scalar, $\beta$ is $1, V$ is normal, $U_{D}=\Phi\left(\frac{V}{\sigma_{\mathcal{E}} \sigma_{V}}\right)$ ). We compare the $I V$ estimand with the policy relevant treatment effect for a policy defined at the base of Table II. If $Z>0$, persons get a bonus $Z t$. Their decision rule for $Z>0$ is $D=\mathbf{1}[Z(1+t)>V]$. People are not forced into participation in the program. Given the assumed distribution of $Z$, and the other parameters of the model, we obtain $h_{P R T E}\left(u_{D}\right)$ as plotted in Figures 3A-3C (the scales differ across the graphs). We use the per capita PRTE and consider 
three instruments. Table III presents estimands for three instruments in the Generalized Roy models for three environments.

The first instrument we consider is $P(Z)$, which ignores the policy $(t)$ effect on choices. It is estimated on a sample with no policy in place. Its weight is plotted in Figure 3A which also displays the $O L S$ weight (discussed later). The $I V$ weights for $P(Z)$ and the weights for $\Delta^{P R T E}$ differ. This is as it should be because $\Delta^{P R T E}$ is making a comparison across regimes but $I V$ in this case is making a comparison within a no policy regime. Given the shape of $\Delta^{M T E}\left(u_{D}\right)$, it is not surprising that the estimand for $I V$ based on $P(Z)$ is so much above the $\Delta^{P R T E}$ which weights a lower-valued segment of $\Delta^{M T E}\left(u_{D}\right)$ more heavily.

The second instrument we consider exploits the variation induced by the policy in place and fits it on samples where the policy is in place. On intuitive grounds, this instrument might be thought to work well for identifying the PRTE, but in fact it does not. The instrument is $\tilde{P}(Z, t)=P(Z(1+t \mathbf{1}[Z>0]))$ which jumps in value when $Z>0$. This is the choice probability in the regime with the policy in place. Figure $3 \mathrm{~B}$ plots the weight for this $I V$ along with the weight for $P(Z)$ as an $I V$ (repeated from $3 \mathrm{~A}$ ). While this weight looks a bit more like the weight for $\Delta^{P R T E}$, it is clearly different.

Figure 3C plots the weight for an ideal instrument for PRTE: a randomization of eligibility. This compares the outcomes in a population with the policy in place with outcomes where it is not. We use an instrument $B$ such that

$$
B= \begin{cases}1 & \text { if a person is eligible to participate in the program } \\ 0 & \text { otherwise. }\end{cases}
$$

Persons for whom $B=1$, make their participation choices under the policy with a jump in $Z, t \mathbf{1}(Z>0)$ in their choice sets. If $B=0$, persons are embargoed from the policy and there is no bonus. This is a prepolicy regime. We assume $\operatorname{Pr}\left[B=1 \mid Y_{0}, Y_{1}, V, Z\right]=\operatorname{Pr}[B=1]=0.5$ so all persons are equally likely to receive or not receive eligibility for the bonus and assignment does not depend on model unobservables in the outcome equation. The Wald estimator in this case is

$$
\frac{E(Y \mid B=1)-E(Y \mid B=0)}{\operatorname{Pr}(D=1 \mid B=1)-\operatorname{Pr}(D=1 \mid B=0)} .
$$


The $I V$ weight for this estimator is a special case of equation (11):

$$
h_{I V}\left(u_{D} \mid B\right)=\frac{E\left(B-E(B) \mid \hat{P}(Z) \geq u_{D}\right) \operatorname{Pr}\left(\hat{P}(Z) \geq u_{D}\right)}{\operatorname{Cov}(B, \hat{P}(Z))},
$$

where $\hat{P}(Z)=P(Z(1+t \mathbf{1}[Z>0]))^{B} P(Z)^{(1-B)}$. Here, the $I V$ is eligibility for a policy and $I V$ is equivalent to a social experiment that identifies the mean gain per participant who switches to participation in the program. It is to be expected that this $I V$ weight and $h_{P R T E}$ are identical.

\section{Monotonicity}

Monotonicity property (iii) is strong. For a general $J(Z)$, there is no guarantee that it will be satisfied even if $J(Z)$ is independent of $\left(Y_{0}, Y_{1}\right)$ given $X$ and if $J(Z)$ is correlated with $D$ given $X=x$ so that standard $I V$ conditions are satisfied. Thus if $Z$ is a $K$-dimensional vector and $J(Z)=Z_{1}$, even if conditional on $Z_{2}=z_{2}, \ldots, Z_{K}=z_{K}, P(Z)$ is monotonic in $Z_{1}$, there is no guarantee that $Z_{1}$ used as an instrument for $D$ has positive weights on the $M T E$.

If we redefine $I V$ for $Z_{1}$ to be conditional on $Z_{2}=z_{2}, \ldots, Z_{K}=z_{K}$, the weights are positive. Conditioning on instruments not used to form the primary covariance relationship is a new concept that does not appear in the conventional $I V$ literature. In conventional cases governed by condition (C-1), any valid instrument identifies the same parameter. In the general case analyzed in this paper, different choices of instruments and the conditioning sets of other $Z$ variables define different parameters.

Figure 4 demonstrates the possibility of negative weights for the model given at its base. In this figure, we use $V$ rather than normalized $F_{V}(V)=U_{D}$ in order to use familiar normal algebra. This simulation is generated from a classical normal error term selection model with nonnormal instruments. The instruments are generated as mixtures of normals from two underlying populations. One can think of this example as a two-component ecological model with different $J(Z), P(Z)$ covariance relationships in the two components. An alternative way of saying the same thing is that there are different $\left(J(Z), \beta^{\prime} Z\right)$ covariance relationships in the two subpopulations generating $D=1\left(\beta^{\prime} Z>V\right)$. In the first component, the covariance between $J(Z)$ and $\beta^{\prime} Z$ is .98 . In the second, the covariance varies as shown in Table IV where the $I V$ is $Z_{1}$ but the choice probability depends on $Z_{1}$ and $Z_{2}\left(\mu_{D}(Z)=\beta^{\prime} Z\right)$. Ceteris paribus, increasing $Z_{1}$ increases the 
probability that $D=1$. Symmetrically, increasing $Z_{2}$ holding $Z_{1}$ constant also increases this probability. Yet, since $Z_{1}$ and $Z_{2}$ covary, varying $Z_{1}$ implicitly varies $Z_{2}$, which may offset the ceteris paribus effect of $Z_{1}$ and produce nonmonotonicity and negative weights. In this example there are different covariance relationships in different normal subcomponents of the data. As $Z_{1}$ increases, $P(Z)$ increases for some people and decreases for other people, leading to two-way flows into and out of treatment for different people. $I V$ estimates the effect of $Z_{1}$ on outcomes not controlling for the other elements of $Z$. For the configuration of parameters shown there (and for numerous other configurations), the $I V$ weight is negative over a substantial range of values.

The negativity of the weights over certain regions exhibited in Figure 4 makes clear that $Z_{1}$ (and more generally $J(Z)$ ) fails the monotonicity condition (iii) and does not estimate a gross treatment effect. Some agents withdraw from participation in the program when $Z_{1}$ is raised (not holding constant $Z_{2}$ ) while others enter, even though ceteris paribus a higher $Z_{1}$ raises participation $(D)$. Thus the widely held view that $I V$ estimates some treatment effect of a change in $D$ induced by a change in $Z_{1}$, is in general false. It estimates a net effect and not a treatment effect, because monotonicity may be violated. Heckman, Urzua, and Vytlacil (2004) present stark examples where MTE is negative, the weights are negative and instrumental variable estimates of treatment effects are positive. Table IV shows how the $I V$ estimand changes with the weights even though the treatment parameters are the same in all three examples.

Monotonicity condition (iii) is testable. Whether condition (iii) corresponds to positive weights on MTE depends on whether all of our assumptions hold, particularly (A-2) and representation (3). If the weights are negative, the change in $J(Z)$ induces two way flows into and out of treatment. Since it is possible to estimate the joint density of $(J(Z), P(Z))$ given $X$ nonparametrically, under our assumptions it is possible to test for the positivity of the weights which under our assumptions is also a test for monotonicity condition (iii). However (A-2) itself is not testable. Monotonicity condition (iii) is distinct from the condition termed "monotonicity" by Imbens and Angrist (1994). We discuss their condition in Section 6. 


\subsection{Policy Relevant Instrumental Variables}

We have just analyzed what $I V$ estimates in terms of weighting MTE. Instead of picking an instrument and hoping that it estimates something interesting, it is more natural to define an economically interesting parameter and see if instrumental variables identify it. Suppose that there is a parameter defined as a weighted average of $\Delta^{M T E}$ conditional on $X=x$. Can we construct a function of $Z$ to use as an ordinary instrument so that the resulting estimand corresponds to the desired weighted average of $\Delta^{M T E}$ ? This question is especially interesting if the estimand is a policy counterfactual. We also consider whether there is any policy counterfactual estimated by a given instrument. We initially consider the case where $P(Z)$ is a continuous random variable. We return at the end of this section to consider the case where the distribution of $P(Z)$ is discrete.

Suppose that we seek to recover a parameter defined by $\int \Delta^{M T E}(x, u) w(u \mid x) d u$ by the method of linear instrumental variables. We know from equation (12) the form of the weights corresponding to the $I V$ estimator for any particular instrument $J(Z)$. We seek an instrument $J(Z)$ that has associated weights on MTE given by equation (12) that are the same as those on the desired parameter

$$
w\left(u_{D} \mid x\right)=\frac{\int_{u_{D}}^{1} T(t \mid x ; J) d F_{P \mid X}(t \mid x)}{\operatorname{Cov}(J, P \mid X=x)}=h_{I V}\left(u_{D} \mid x, J\right)
$$

Assuming that $F_{P \mid X}$ has a density with respect to Lebesgue measure, the second term in this expression is differentiable in $u$ (almost everywhere). Assuming that $w\left(u_{D} \mid x\right)$ is also differentiable at all points of evaluation, it follows that $w^{\prime}\left(u_{D} \mid x\right)=-\frac{T\left(u_{D} \mid x ; J\right) f_{P \mid X}\left(u_{D} \mid x\right)}{\operatorname{Cov}(J, P \mid X=x)}$. The following proposition provides conditions under which an instrument exists with the desired properties.

Proposition 1. Under the following conditions

(i) $F_{P \mid X}(\cdot)$ has a density with respect to Lebesgue measure;

(ii) $w(\cdot \mid x)$ satisfies the following properties: $w\left(u_{D} \mid x\right)$ differentiable in $u_{D}$ for all $u_{D} \in[0,1], \int_{0}^{1} w\left(u_{D} \mid x\right) d u_{D}=$ 1 , and $w(1 \mid x)=w(0 \mid x)=0 ;$

(iii) $f_{P \mid X}(t \mid x)=0$ implies $w^{\prime}(t \mid x)=0$; 
there exists an instrument $J_{x}(Z)$ such that $\operatorname{Cov}\left(J_{x}, D \mid X=x\right) \neq 0$ and $w\left(u_{D} \mid x\right)=h_{I V}\left(u_{D} \mid x, J_{x}\right)$. An instrument that satisfies these conditions is ${ }^{34}$

$$
J_{x}(Z)= \begin{cases}\frac{w^{\prime}(P(Z) \mid x)}{f_{P \mid X}(P(Z) \mid x)} & \text { if } f_{P \mid X}(P(Z) \mid x)>0 \\ 0 & \text { if } f_{P \mid X}(P(Z) \mid x)=0 .{ }^{35}\end{cases}
$$

Under (i), conditions (ii) and (iii) are necessary and sufficient for the existence of such an instrument.

Proof. See Appendix C.

Q.E.D.

Condition (i) is a regularity condition requiring that $P(Z)$ be a continuous random variable. We examine the case where $P(Z)$ is discrete at the end of this section. Condition (ii) requires that the desired weights on $M T E$ be proper weights in the sense of integrating up to one, and also that the weights satisfy the regularity conditions that the weights are differentiable and that the weights are zero when evaluated at the $u_{D}$ values of zero and one. The first and third of these conditions mimic the properties of any $I V$ weights, and the second condition mimics the property of any $I V$ weights when the instrument is continuous. Condition (iii) is a strong, but natural, condition. It requires that the support of the propensity score includes the support of $w^{\prime}(\cdot \mid x)$. Thus, the density of the propensity score has to be positive at any evaluation point where $w^{\prime}(\cdot \mid x)$ is nonzero. This condition will always be satisfied if $f_{P \mid X}(t \mid x)>0$ for all $t \in[0,1]$. Given (i), if (ii) or (iii) fails, then no instrument exists that provides the desired weights. If the desired weights do not integrate to one or are not differentiable, then as long as $P(Z)$ is continuous (has a distribution that is absolutely continuous with respect to Lebesgue measure), there will not exist any instrument that provides the desired weights. If the weights are nonconstant over an interval outside of the support of the propensity score, then no such instrument can be constructed that would provide the desired weights.

One implication of Proposition 1 is that if $P(Z)$ is continuous, when the $M T E$ depends on $u_{D}$ in a nontrivial way there does not exist any instrument that provides the weights corresponding to ATE or TT. To see this, recall that the weights for $A T E$ and $T T$ do not satisfy $w(1 \mid x)=w(0 \mid x)=0$, so that the

\footnotetext{
${ }^{34}$ When such an instrument exists, it will not be unique, since the $I V$ estimand will be invariant to rescaling or location shifts for the instrument.

${ }^{35}$ Note that $f_{P \mid X}(P(Z) \mid x)>0$ with probability 1 so that $J_{x}(Z)=w^{\prime}(P(Z) \mid x) / f_{P \mid X}(P(Z) \mid x)$ with probability 1.
} 
weights for $A T E$ and $T T$ do not satisfy condition (ii) of the proposition. Thus, under assumptions (A-1)(A-5) and under the additional assumptions of the proposition, no instrument exists giving the weights for $A T E$ or $T T$ if $P(Z)$ is a continuous random variable. This statement leaves open the question of whether instruments will exist that answer policy counterfactuals. We now specialize the previous proposition for the special case of policy weights, using the notation for policy counterfactuals from Section 3.

\section{Proposition 2. Assume}

(i) $F_{P \mid X}(\cdot), F_{P_{a^{\prime}} \mid X}(\cdot)$ and $F_{P_{a} \mid X}(\cdot)$ have densities with respect to Lebesgue measure where $P$ is the initial (benchmark) probability and $P_{a^{\prime}}$ and $P_{a}$ are the probabilities associated with two policies (possibly) distinct from the benchmark policy.

(ii) $E\left(P_{a} \mid X=x\right) \neq E\left(P_{a^{\prime}} \mid X=x\right)$;

(iii) for any $t, f_{P \mid X}(t \mid x)=0$ implies $f_{P_{a} \mid X}(t \mid x)-f_{P_{a^{\prime}} \mid X}(t \mid x)=0$.

Define $J_{x}$ to be a policy relevant instrument if it satisfies $\operatorname{Cov}\left(J_{x}, D \mid X=x\right) \neq 0$ and

$$
h_{I V}\left(u_{D} \mid x, J_{x}\right)=\frac{\int_{u_{D}}^{1} T\left(t \mid x ; J_{x}\right) d F_{P \mid X}(t \mid x)}{\operatorname{Cov}\left(J_{x}, P \mid X=x\right)}=\frac{F_{P_{a^{\prime}} \mid X}(t \mid x)-F_{P_{a} \mid X}(t \mid x)}{E\left(P_{a^{\prime}} \mid X=x\right)-E\left(P_{a} \mid X=x\right)} .
$$

Given condition (i) and (ii), condition (iii) is necessary and sufficient for the existence of such an instrument. If the instrument exists, it can be constructed as: ${ }^{36}$

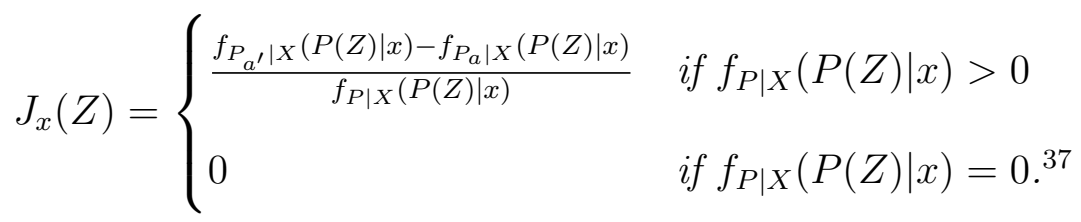

Proof. Follows by verifying the conditions of Proposition 1. See Appendix C.

Q.E.D.

Condition (i) requires that the propensity score be a continuous random variable in a benchmark regime and under both alternative regimes. Condition (ii) requires that a fraction of individuals selecting into treatment under regime $a$ is different than the fraction under regime $a^{\prime}$. Condition (iii) imposes the

\footnotetext{
${ }^{36}$ If such a $J_{x}(Z)$ exists, then any linear function of $J_{x}(Z)$ will also produce the desired set of weights.

${ }^{37}$ Note that $f_{P \mid X}(P(Z) \mid x)>0$ with probability 1 so that $J_{x}(Z)=\frac{\left(f_{P_{a^{\prime}} \mid X}(P(Z) \mid x)-f_{P_{a} \mid X}(P(Z) \mid x)\right)}{f_{P \mid X}(P(Z) \mid x)}$ with probability 1 .
} 
requirement that the densities of the propensity score in the two regimes only differ at evaluation points in the support of the benchmark propensity score. If (iii) fails, then no policy relevant instrument can be constructed.

An immediate corollary of the proposition is that $I V$ using the propensity score as the instrument recovers the policy relevant parameter if

$$
P(Z)=\alpha(X)+\beta(X)\left[\frac{f_{P_{a^{\prime}} \mid X}(P(Z))-f_{P_{a} \mid X}(P(Z))}{f_{P \mid X}(P(Z))}\right]
$$

where $\alpha(X)=E(P(Z) \mid X)$ and $\beta(X)=-\operatorname{Var}(P(Z) \mid X)$, i.e. only if the propensity score is linear in $\left\{f_{P_{a^{\prime}} \mid X}(P(Z))-f_{P_{a} \mid X}(P(Z))\right\} / f_{P \mid X}(P(Z))$.

A related question asks whether, given an instrument, there exists a policy counterfactual such that the given instrument is the policy relevant instrument for that counterfactual. We investigate this question for policy counterfactuals starting from a benchmark distribution of $P(Z)$ (the benchmark policy is $a$ so $\left.P_{a}\left(Z_{a}\right)=P(Z)\right)$ to some new policy characterized by $P_{a^{\prime}}\left(Z_{a^{\prime}}\right)$. We first answer the question for the special case where the propensity score is the instrument. Solving for $f_{P_{a^{\prime}} \mid X}(P(Z))$ in $(13)$, the propensity score will be the policy relevant instrument for a policy characterized by

$$
f_{P_{a^{\prime}} \mid X}\left(u_{D}\right)=f_{P \mid X}\left(u_{D}\right)\left(1-\frac{u_{D}-E(P(Z) \mid X)}{\operatorname{Var}(P(Z) \mid X)}\right) .
$$

If $f_{P_{a^{\prime}} \mid X}$ given by equation (14) is a proper density, then instrumental variables using the propensity score directly estimates the effect of a policy intervention that changes the density of the propensity score from $f_{P \mid X}$ to $f_{P_{a^{\prime}} \mid X}$ where $f_{P_{a^{\prime}} \mid X}$ is given by equation $(14)$. To be a proper density, $f_{P_{a^{\prime}} \mid X}$ must integrate to one and be nonnegative for all evaluation points. $f_{P_{a^{\prime}} \mid X}(\cdot)$ integrates to one. ${ }^{38} f_{P_{a^{\prime}} \mid X}(\cdot)$ will be nonnegative and thus a proper density if and only if $u_{D}-E(P(Z) \mid X) \leq \operatorname{Var}(P(Z))$ for all $u_{D}$ such that $f_{P \mid X}\left(u_{D}\right)>0$. If we let $p_{x}^{\mathrm{Max}}$ denote the maximum of the support of $P(Z)$ conditional on $X$, we can rewrite this condition as $p_{x}^{\operatorname{Max}}-E(P(Z) \mid X=x) \leq \operatorname{Var}(P(Z) \mid X=x)$. Nothing guarantees that this condition holds so one cannot guarantee that an instrument produces any policy counterfactual. Not all instruments answer well posed policy questions.

\footnotetext{
${ }^{38} \int f_{P \mid X}\left(u_{D}\right)\left(1-\frac{u_{D}-E(P(Z) \mid X)}{\operatorname{Var}(P(Z) \mid X)}\right) d u_{D}=1$ since $\int u_{D} f_{P \mid X}\left(u_{D}\right) d u_{D}=E(P(Z) \mid X)$
} 
We next consider the question of whether a general instrument is the policy relevant instrument for some policy. Following the same series of steps used to establish (14), if the instrument $J(Z)$ answers a corresponding policy question, then the policy imposes the restriction that

$$
f_{P_{a^{\prime}} \mid X}\left(u_{D}\right)=f_{P \mid X}\left(u_{D}\right)\left(1-\frac{E\left(J(Z) \mid X, P(Z)=u_{D}\right)-E(J(Z) \mid X)}{\operatorname{Cov}(J, P(Z) \mid X)}\right)
$$

The implied $f_{P_{a^{\prime}}}(\cdot)$ integrates to one. It is nonnegative for all evaluation points if and only if

$$
\frac{E\left(J(Z) \mid X, P(Z)=u_{D}\right)-E(J(Z) \mid X)}{\operatorname{Cov}(J, P(Z) \mid X)} \leq 1
$$

for all $u_{D}$ such that $f_{P \mid X}\left(u_{D}\right)>0$. If this condition fails, the instrument is not the policy relevant instrument for any policy. Nothing in the structure of the problem imposes this requirement.

The preceding analysis conditions on $X$. Suppose that we wish to recover unconditional parameters, e.g., those defined by $\int\left[\int \Delta^{M T E}(x, u) w(u \mid x) d u\right] d F_{X}(x)$. If the conditions of Proposition 1 hold for $X=x$ (almost everywhere), then one solution would be to construct $J_{x}(Z)$ for each $x$, estimate the parameter conditional on $X$ for each $x$, and then average over $x$ values. However, from the construction of $J_{x}(Z)$, one can use instrumental variables unconditional on $X$ with the constructed $J(Z)$ as the instrument to obtain the desired parameter in one step.

Proposition 3. Assume that the conditions of Proposition 1 hold for almost all X. Construct

$$
J(Z)= \begin{cases}\frac{w^{\prime}(P(Z) \mid X)}{f_{P \mid X}(P(Z))} & \text { if } f_{P \mid X}(P(Z))>0 \\ 0 & \text { if } f_{P \mid X}(P(Z))=0 .{ }^{39}\end{cases}
$$

Then

$$
\frac{\operatorname{Cov}(J(Z), Y)}{\operatorname{Cov}(J(Z), D)}=\int\left[\int \Delta^{\mathrm{MTE}}\left(x, u_{D}\right) w\left(u_{D} \mid x\right) d u\right] d F_{X}(x) .
$$

Proof. See Appendix C.

Thus far we have considered the case where $P(Z)$ is a continuous random variable. Is it possible to construct an instrument that produces the desired weights if $P(Z)$ is discrete? The following proposition

\footnotetext{
${ }^{39}$ Note that $f_{P \mid X}(P(Z) \mid X)>0$ with probability 1 so that $J(Z)=w^{\prime}(P(Z) \mid X) / f_{P \mid X}(P(Z) \mid X)$ with probability 1.
} 
shows that instrumental variables estimators are only able to produce a very narrow range of weights if $P(Z)$ is discrete. In particular, they only produce weights given by step functions with the jumps in the weight function occurring only at the support points of $P(Z)$.

Proposition 4. Under the following conditions

(i) The support of the distribution of $P(Z)$ conditional on $X$ is a finite set, $\left\{p_{1}, \ldots, p_{K}\right\}$ with $p_{1}<p_{2}<$ $\ldots<p_{K}$ and with $\operatorname{Pr}\left[P(Z)=p_{j} \mid X=x\right]>0$ for each $j=1, \ldots, K$;

(ii) $w(\cdot \mid x)$ satisfies the following properties: $\int_{0}^{1} w\left(u_{D} \mid x\right) d u=1$, and $w\left(u_{D} \mid x\right)=0$ for $u_{D} \leq p_{1}$ and for $u_{D}>p_{K}$

(iii) $w\left(u_{D} \mid x\right)$ is constant in $u$ over the interval $\left(p_{j}, p_{j+1}\right]$ for $j=1, \ldots, K$;

there exists an instrument $J_{x}(Z)$ such that $\operatorname{Cov}\left(J_{x}, D \mid X=x\right) \neq 0$ and

$$
w\left(u_{D} \mid x\right)=\frac{\int_{u_{D}}^{1} T\left(t \mid x ; J_{x}\right) d F_{P \mid X}(t \mid x)}{\operatorname{Cov}\left(J_{x}, P \mid X=x\right)} .
$$

An instrument that satisfies these conditions is

$$
J_{x}(Z)=\sum_{j=1}^{K} \frac{1}{\operatorname{Pr}\left[P(Z)=p_{j} \mid X=x\right]}\left(w_{j}-w_{j+1}\right) \mathbf{1}\left[P(Z)=p_{j}\right],
$$

where $w_{j}$ denotes the (constant) value of $w\left(u_{D} \mid x\right)$ over the interval $\left(p_{j-1}, p_{j}\right]$ for $j=2, \ldots, K-1$, and where $w_{1}=w_{K+1}=0 .{ }^{40}$ Given condition (i), conditions (ii) and (iii) are necessary and sufficient for the existence of such an instrument.

Proof. See Appendix C.

Thus, if $P(Z)$ is a discrete random variable, only a very limited set of possible weights on $P(Z)$ can be captured through proper choice of the instrument so the class of policies that can be generated by $I V$ is very limited. We next use our framework to analyze the $O L S$ estimator and the assumptions about $\Delta^{M T E}$ imposed in one widely used version of the method of matching.

\footnotetext{
${ }^{40}$ When such an instrument exists, it will not be unique, since the $I V$ estimand will be invariant to rescaling or location shifts for the instrument.
} 


\subsection{OLS Weights and Matching}

The $O L S$ estimator can also be represented as a weighted average of $\triangle^{M T E}$. The weight is given in Table IB where $U_{1}$ and $U_{0}$ are defined as deviations from conditional expectations, $U_{1}=Y_{1}-E\left(Y_{1} \mid X\right)$, $U_{0}=Y_{0}-E\left(Y_{0} \mid X\right)$. Unlike the weights for $\Delta^{T T}$ and $\Delta^{A T E}$, these weights do not necessarily integrate to one and they are not necessarily nonnegative. The $O L S$ weights for the Generalized Roy Model are plotted in Figure 1B. The negative component of the $O L S$ weight leads to a smaller $O L S$ treatment estimate compared to the other treatment effects in Table II.

Table II shows the estimated $O L S$ treatment effect for the Generalized Roy example. For a binary regressor $D, O L S$ conditional on $X$ identifies

$$
\begin{aligned}
\Delta^{O L S}(X) & =E\left(Y_{1} \mid X, D=1\right)-E\left(Y_{0} \mid X, D=0\right) \\
& =E\left(Y_{1}-Y_{0} \mid X, D=1\right)+\left\{E\left(Y_{0} \mid X, D=1\right)-E\left(Y_{0} \mid X, D=0\right)\right\}
\end{aligned}
$$

where the term in braces is the "selection bias" term - the difference in pretreatment outcomes between treated and untreated individuals. It is also the bias for $\Delta^{T T}$. The large negative selection bias in this example is consistent with comparative advantage as emphasized by Roy (1951). People who are good in sector 1 (i.e. treatment) may be very poor in Sector 0 (no treatment). The differences among the policy relevant treatment effects, the conventional treatment effects and the $O L S$ estimand are illustrated in Figure $3 \mathrm{~A}$ and Tables II and III. As is evident from Table II, it is not at all clear that the instrumental variable estimator, with instruments that satisfy classical properties, performs better than $O L S$ in identifying the policy relevant treatment effect.

If there is no selection conditional on covariates, $U_{D} \Perp\left(Y_{1}, Y_{0}\right) \mid X$, then $E\left(U_{1} \mid X, U_{D}\right)=E\left(U_{1} \mid\right.$ $X)=0$ and $E\left(U_{0} \mid X, U_{D}\right)=E\left(U_{0} \mid X\right)=0$ so that the $O L S$ weights are unity and $O L S$ identifies ATE. OLS is a form of matching. Furthermore, $U_{D} \Perp\left(Y_{1}, Y_{0}\right) \mid X$ implies that $\Delta^{M T E}\left(x, u_{D}\right)$ does not vary with $u_{D}$, i.e., $\Delta^{M T E}\left(X, u_{D}\right)=\Delta^{M T E}\left(X, u_{D}^{\prime}\right)$ for $u_{D}, u_{D}^{\prime}$ (almost everywhere) so all treatment effects are the same. Observe that given the assumed conditional independence in terms of $X$, we can identify $A T E$ and $T T$ without requiring a $Z$ satisfying (A-2). If there is such a $Z$, the conditional independence condition implies under (A-1)-(A-5) that $E(Y \mid X, P(Z)=p)$ is linear in $p$. This conditional independence assumption is invoked in the method of matching and has come into wide spread use. The method is based 
upon the assumption that there is no purposeful selection into the program based on unmeasured (by the econometrician) components of gain ${ }^{41}$.

One can weaken the assumption that $U_{D} \Perp\left(Y_{1}, Y_{0}\right) \mid X$ to the condition that $Y_{1}$ and $Y_{0}$ are mean independent of $D$ conditional on $X{ }^{42}$ However, $D$ will be mean independent of $Y_{1}, Y_{0}$ conditional on $X$ without $U_{D}$ being independent of $Y_{1}, Y_{0}$ conditional on $X$ only if fortuitous balancing occurs with regions of positive $Y_{1}, Y_{0}$ dependence on $U_{D}$ and regions of negative $Y_{1}, Y_{0}$ dependence on $U_{D}$ just exactly offsetting each other. Such balancing is ruled out in the Roy model and in the Generalized Roy Model. ${ }^{43,44}$ We next apply our framework to analyze policy forecasting problems.

\section{Out of Sample Policy Forecasting, Forecasting the Effects of}

\section{New Policies and Structural Models Based on the $M T E$}

Section 3 introduced the concept of the Policy Relevant Treatment Effect and invokes a policy invariance assumption. In this section, we present conditions for constructing PRTE for new environments and for new programs using historical data.

Using the terminology of Campbell and Stanley (1966), estimating the impact of a program in place in a particular environment is the problem of "internal validity." Extrapolating internally valid estimates to new environments, "external validity," or forecasting the effects of new policies, are also important problems which we now address.

Let $a \in \mathcal{A}$ denote a policy characterized by random vector $Z_{a}$. Let $e \in \mathcal{E}$ denote an environment

${ }^{41}$ See Heckman and Navarro (2004) and Heckman and Vytlacil (2005)for a more extensive discussion of matching estimators.

${ }^{42}$ See Heckman, Ichimura, Smith, and Todd (1998) and Heckman, Ichimura, and Todd (1997). If the goal of the analysis is to estimate $\Delta^{T T}$, one can get by with the weaker assumption that only $Y_{0}$ is mean independent of $D$ conditional on $X$ since $E\left(Y_{1} \mid D=1, X=x\right)$ is identified from observational data so there can be selection arising from dependence between $Y_{1}$ and $D$.

${ }^{43}$ In particular, assume $Y_{j}=\mu_{j}(X)+U_{j}$ for $j=0,1$, assume $D=\mathbf{1}\left[Y_{1}-Y_{0} \geq C(Z)+U_{C}\right]$, and let $U_{D}=U_{C}-\left(U_{1}-U_{0}\right)$. Then if $U_{C} \Perp\left(U_{1}-U_{0}\right)$, and $U_{C}$ has a log concave density, then $E\left(Y_{1}-Y_{0} \mid X, U_{D}=u_{D}\right)$ is decreasing in $u_{D}, \Delta^{T T}(x)>\Delta^{A T E}(x)$, and the matching conditions do not hold. If $U_{C} \Perp\left(U_{1}-U_{0}\right)$ but $U_{C}$ does not have a log concave density, then it is still the case that $\left(U_{1}-U_{0}, U_{D}\right)$ is negative quadrant dependent. One can show that $\left(U_{1}-U_{0}, U_{D}\right)$ being negative quadrant dependent implies that $\Delta^{T T}(x)>\Delta^{A T E}(x)$, and thus again that the matching conditions cannot hold. See Heckman and Vytlacil (2005) for further discussion.

${ }^{44}$ It is sometimes said that the matching assumptions are "for free" (See Gill and Robins, 2001) because one can always replace unobserved $F\left(Y_{1} \mid X=x, D=0\right)$ with $F\left(Y_{1} \mid X=x, D=1\right)$ and unobserved $F\left(Y_{0} \mid X=x, D=1\right)$ with $F\left(Y_{0} \mid X=x, D=0\right)$. This ignores the counterfactual states generated under the matching assumptions that (C-1) is true in the population. The assumed absence of selection is not a "for free" assumption, and produces fundamentally different counterfactual states for the same model under matching and selection assumptions. 
characterized by random vector $X_{e}$. A history, $\mathcal{H}$, is a collection of policy-environment $(a, e)$ pairs that have been experienced and documented. We assume that the environment is autonomous so the choice of $a$ does not affect $X_{e}$. Letting $X_{e, a}$ denote the value of $X_{e}$ under policy $a$, autonomy requires that

(A-8) $X_{e, a}=X_{e} \quad \forall a, e \quad$ (autonomy).

Autonomy is a more general notion than the concept introduced in (A-6). They are the same when the policy is a treatment. General equilibrium feedback effects can cause a failure of autonomy. In this section we will assume autonomy, in accordance with the partial equilibrium tradition in the treatment effect literature. ${ }^{45}$

Evaluating a particular policy $a^{\prime}$ in environment $e^{\prime}$ is straightforward if $\left(a^{\prime}, e^{\prime}\right) \in \mathcal{H}$. One simply looks at the associated outcomes and treatment effects formed in that policy environment and applies the methods previously discussed to obtain internally valid estimates. The challenge comes in forecasting the impacts of policies $\left(a^{\prime}\right)$ in environments $\left(e^{\prime}\right)$ for $\left(a^{\prime}, e^{\prime}\right)$ not in $\mathcal{H}$.

We show how $\Delta^{M T E}$ plays the role of a policy-invariant functional that aids in creating counterfactual states never previously experienced. We focus on the problem of constructing the policy relevant treatment effect $\triangle^{P R T E}$ but our discussion applies more generally to the other treatment parameters.

Given the assumptions invoked in Section 3, $\Delta^{M T E}$ can be used to evaluate a whole menu of policies characterized by different conditional distributions of $P_{a^{\prime}}$. In addition, given our assumptions, we can focus on how policy $a^{\prime}$, which is characterized by $Z_{a^{\prime}}$, produces the distribution $F_{P_{a^{\prime}} \mid X}$ which weights an invariant $\Delta^{M T E}$ without having to conduct a new investigation of $(Y, X, Z)$ relationships for each proposed policy. ${ }^{46}$

\subsection{Constructing Weights for New Policies in a Common Environment}

The problem of constructing $\Delta^{P R T E}$ for policy $a^{\prime}$ (compared to $\bar{a}$ ) in environment $e$ when $\left(a^{\prime}, e\right) \notin \mathcal{H}$ entails constructing $E\left(V\left(Y_{a^{\prime}}\right)\right)$. We maintain the assumption that the baseline policy is observed, so $(\bar{a}, e) \in \mathcal{H}$. We assume (A-1)-(A-5), (A-7) and (A-8) and use (3) to characterize choices. The policy does not change the distribution of $\left(Y_{0}, Y_{1}, U_{D}\right)$ conditional on $X$. Under these conditions, equation (6) is a valid expression

\footnotetext{
${ }^{45}$ However, see Heckman, Lochner, and Taber (1998) for an example of a nonautonomous treatment model.

${ }^{46}$ Ichimura and Taber (2002) present a discussion of local policy analysis in a more general framework without the MTE structure, using a framework developed by Hurwicz (1962).
} 
for PRTE and constructing PRTE only requires identification of $\Delta^{M T E}$ and constructing $F_{P_{a^{\prime}} \mid X_{e}}$ from the policy histories $\mathcal{H}_{e}$, defined as the elements of $\mathcal{H}$ for a particular environment $e, \mathcal{H}_{e}=\{a:(a, e) \in \mathcal{H}\}$.

Associated with the policy histories $a \in \mathcal{H}_{e}$ is a collection of policy variables $\left\{Z_{a}: a \in \mathcal{H}_{e}\right\}$. Suppose that a new policy $a^{\prime}$ can be written as $Z_{a^{\prime}}=T_{a^{\prime}, j}\left(Z_{j}\right)$ for some $j \in \mathcal{H}_{e}$ where $T_{a^{\prime}, j}$ is a known deterministic transformation and $Z_{a^{\prime}}$ has the same list of variables as $Z_{j}$. Examples of policies that can be characterized in this way are tax and subsidy policies on wages, prices and incomes that affect unit costs (wages or prices) and transfers. Tuition might be shifted upward for everyone by the same amount, or tuition might be shifted according to a nonlinear function of current tuition, parents' income, and other observable characteristics in $Z_{j}$.

Constructing $F_{P_{a^{\prime}} \mid X_{e}}$ from data in the policy history entails two distinct steps. From the definitions, $\operatorname{Pr}\left(P_{a^{\prime}} \leq t \mid X_{e}\right)=\operatorname{Pr}\left(Z_{a^{\prime}}: \operatorname{Pr}\left(D_{a^{\prime}}=1 \mid Z_{a^{\prime}}, X_{e}\right) \leq t \mid X_{e}\right)$. If (i) we know the distribution of $Z_{a^{\prime}}$, and (ii) we know the function $\operatorname{Pr}\left(D_{a^{\prime}}=1 \mid Z_{a^{\prime}}=z, X_{e}=x\right)$ over the appropriate support, we can then recover the distribution of $P_{a^{\prime}}$ conditional on $X_{e}$. Given that $Z_{a^{\prime}}=T_{a^{\prime}, j}\left(Z_{j}\right)$ for a known function $T_{a^{\prime}, j}(\cdot)$, step (i) is straightforward since we recover the distribution of $Z_{a^{\prime}}$ from the distribution of $Z_{j}$ by using the fact that $\operatorname{Pr}\left(Z_{a^{\prime}} \leq t \mid X_{e}\right)=\operatorname{Pr}\left(Z_{j}: T_{a^{\prime}, j}\left(Z_{j}\right) \leq t \mid X_{e}\right)$. Alternatively, part of the specification of the policy $a^{\prime}$ might be the distribution $\operatorname{Pr}\left(Z_{a^{\prime}} \leq t \mid X_{e}\right)$. We now turn to the second step, recovering the function $\operatorname{Pr}\left(D_{a^{\prime}}=1 \mid Z_{a^{\prime}}=z, X_{e}=x\right)$ over the appropriate support.

If $Z_{a^{\prime}}$ and $Z_{j}$ contain the same elements though possibly with different distributions, then a natural approach to forecasting the new policy is to postulate that

$$
\begin{aligned}
P_{j}(z) & =\operatorname{Pr}\left(D_{j}=1 \mid Z_{j}=z, X_{e}\right) \\
& =\operatorname{Pr}\left(D_{a^{\prime}}=1 \mid Z_{a^{\prime}}=z, X_{e}\right)=P_{a^{\prime}}(z)
\end{aligned}
$$

i.e. that over a common support for $Z_{j}$ and $Z_{a^{\prime}}$ the known conditional probability function and the desired conditional probability function agree. Condition (15) will hold, for example, if $D_{j}=\mathbf{1}\left[\mu_{D}\left(Z_{j}\right)-U_{D} \geq 0\right]$, $D_{a^{\prime}}=\mathbf{1}\left[\mu_{D}\left(Z_{a^{\prime}}\right)-U_{D} \geq 0\right], Z_{j} \Perp U_{D} \mid X_{e}$, and $Z_{a^{\prime}} \Perp U_{D} \mid X_{e}$. Even if condition (15) is satisfied on a common support, the support of $Z_{j}$ and $Z_{a^{\prime}}$ may not be the same. If the support of the distribution of $Z_{a^{\prime}}$ is not contained in the support of the distribution of $Z_{j}$, then some form of extrapolation is needed. Alternatively, if we strengthen our assumptions so that $(15)$ holds for all $j \in \mathcal{H}_{e}$, we can identify $P_{a^{\prime}}(z)$ for 
all $z$ in $\bigcup_{j \in \mathcal{H}_{e}} \operatorname{Supp}\left(Z_{j}\right)$. However, there is no guarantee that the support of the distribution of $Z_{a^{\prime}}$ will be contained in $\bigcup_{j \in \mathcal{H}_{e}} \operatorname{Supp}\left(Z_{j}\right)$, in which case some form of extrapolation is needed.

If extrapolation is required, then one approach is to assume a parametric functional form for $P_{j}(\cdot)$. Given a parametric functional form, one can use the joint distribution of $\left(D_{j}, Z_{j}\right)$ to identify the unknown parameters of $P_{j}(\cdot)$ and then extrapolate the parametric functional form to evaluate $P_{j}(\cdot)$ for all evaluation points in the support of $Z_{a^{\prime}}$. Alternatively, if there is overlap between the support of $Z_{a^{\prime}}$ and $Z_{j},{ }^{47}$ so there is some overlap in the historical and policy $a^{\prime}$ supports of $Z$, we may use nonparametric methods presented in Matzkin (1994) with functional restrictions (e.g. homogeneity) to construct the desired probabilities on new supports or to bound them. Under the appropriate conditions, we may use analytic continuation to extend $\operatorname{Pr}\left(D_{j}=1 \mid Z_{j}=z, X_{e}=x\right)$ to a new support for each $X_{e}=x$ (Rudin, 1974).

The approach just presented is based on the assumption stated in equation (15). That assumption is quite natural when $Z_{a^{\prime}}$ and $Z_{j}$ both contain the same elements, say they both contain tuition and parent's income. However, in some cases $Z_{a^{\prime}}$ might contain additional elements not contained in $Z_{j}$. As an example, $Z_{a^{\prime}}$ might include new user fees while $Z_{j}$ consists of taxes and subsidies but does not include user fees. In this case, the assumption stated in equation (15) is not expected to hold and is not even well defined if $Z_{a^{\prime}}$ and $Z_{j}$ contain a different number of elements.

A more basic approach analyzes a class of policies that operate on constraints, prices and endowments arrayed in vector $C$. Given the preferences and technology of the agent, a given $C=c$, however arrived at, generates the same choices for the agent. Thus a wage tax offset by a wage subsidy of the same amount produces a wage that has the same effect on choices as a no-policy wage. Policy $j$ affects $C$ (e.g. it affects prices paid, endowments and constraints). Define a map $\Phi_{j}: Z_{j} \longrightarrow C_{j}$ which maps a policy $j$, described by $Z_{j}$, into its consequences $\left(C_{j}\right)$ for the baseline, fixed-dimensional vector $C$. A new policy $a^{\prime}$, characterized by $Z_{a^{\prime}}$, produces $C_{a^{\prime}}$ that is possibly different from $C_{j}$ for all previous policies $j \in \mathcal{H}_{e}$.

\footnotetext{
${ }^{47}$ If we strengthen condition (15) to hold for all $j \in \mathcal{H}_{e}$, then the condition becomes that $\operatorname{Supp}\left(Z_{a^{\prime}}\right) \cap \bigcup_{j \in \mathcal{H}_{e}} \operatorname{Supp}\left(Z_{j}\right)$ is not empty.
} 
To construct the random variable $P_{a^{\prime}}=\operatorname{Pr}\left(D_{a^{\prime}}=1 \mid Z_{a^{\prime}}, X_{e}\right)$, we postulate that

$$
\begin{aligned}
\operatorname{Pr}\left(D_{j}=1 \mid Z_{j} \in \Phi_{j}^{-1}(c), X_{e}=x\right) & =\operatorname{Pr}\left(D_{j}=1 \mid C_{j}=c, X_{e}=x\right) \\
& =\operatorname{Pr}\left(D_{a^{\prime}}=1 \mid C_{a^{\prime}}=c, X_{e}=x\right) \\
& =\operatorname{Pr}\left(D_{a^{\prime}}=1 \mid Z_{a^{\prime}} \in \Phi_{a^{\prime}}^{-1}(c), X_{e}=x\right),
\end{aligned}
$$

where $\Phi_{j}^{-1}(c)=\left\{z: \Phi_{j}(z)=c\right\}$ and $\Phi_{a^{\prime}}^{-1}(c)=\left\{z: \Phi_{a^{\prime}}(z)=c\right\}$. Given these assumptions, our ability to recover $\operatorname{Pr}\left(D_{a^{\prime}}=1 \mid Z_{a^{\prime}}=z, X_{e}=x\right)$ for all $(z, x)$ in the support of $\left(Z_{a^{\prime}}, X_{e}\right)$ depends on what $\Phi_{j}$ functions have been historically observed and how rich the histories of $C_{j}, j \in \mathcal{H}_{e}$ are. For each $z_{a^{\prime}}$ evaluation point in the support of the distribution of $Z_{a^{\prime}}$, there is a corresponding $c=\Phi_{a^{\prime}}\left(z_{a^{\prime}}\right)$ evaluation point in the support of the distribution of $C_{j}=\Phi_{j}\left(Z_{j}\right)$. If, in the policy histories, there is at least one $j \in \mathcal{H}_{e}$ such that $\Phi_{j}\left(z_{j}\right)=c$ for a $z_{j}$ with $\left(z_{j}, x\right)$ in the support of the distribution of $\left(Z_{j}, X_{e}\right)$ then we can construct the probability of the new policy from data in the policy histories. The methods used to extrapolate $P_{a^{\prime}}(\cdot)$ over new regions, discussed previously, apply here. If the distribution of $C_{a^{\prime}}$ (or $\Phi_{a^{\prime}}$ and the distribution of $Z_{a^{\prime}}$ ) is known as part of the specification of the proposed policy, the distribution of $F_{P_{a^{\prime}} \mid X_{e}}$ can be constructed using the constructed $P_{a^{\prime}}$. Alternatively, if we can relate $C_{a^{\prime}}$ to $C_{j}$ by $C_{a^{\prime}}=\Psi_{a^{\prime}, j}\left(C_{j}\right)$ for a known function $\Psi_{a^{\prime}, j}$ or if we can relate $Z_{a^{\prime}}$ to $Z_{j}$ by $Z_{a^{\prime}}=T_{a^{\prime}, j}\left(Z_{j}\right)$ for a known function $T_{a^{\prime}, j}$, and the distributions of $C_{j}$ and/or $Z_{j}$ are known for some $j \in \mathcal{H}_{e}$, we can apply the method previously discussed to derive $F_{P_{a^{\prime}} \mid X_{e}}$ and hence the policy weights for the new policy.

This approach assumes that a new policy acts on components of $C$ like a policy in $\mathcal{H}_{e}$, so it is possible to forecast the effect of a policy with nominally new aspects. The essential idea is to recast the new aspects of policy in terms of old aspects previously measured. Thus in a model of schooling, let $D=$ $\mathbf{1}\left[Y_{1}-Y_{0}-B \geq 0\right]$ where $Y_{1}-Y_{0}$ is the discounted gain in earnings from going to school and $B$ is the tuition cost. Here the effect of cost is just the negative of the effect of return. Historically, we might only observe variation in $Y_{1}-Y_{0}$ (say tuition has never previously been charged). But $B$ is on the same footing (has the same effect on choice, except for sign) as $Y_{1}-Y_{0}$. This identified historical variation in $Y_{1}-Y_{0}$ can be used to nonparametrically forecast the effect of introducing $B$, provided that the support of $P_{a^{\prime}}$ is in the historical support generated by the policy histories in $\mathcal{H}_{e}$. Otherwise, some functional structure (parametric or semi-parametric) must be imposed to solve the support problem for $P_{a^{\prime}}$. 
As another example, following Marschak (1953), consider the introduction of wage taxes in a world where there has never before been a tax. Let $Z_{j}$ be the wage without taxes. We seek to forecast a post-tax net wage $Z_{a^{\prime}}=(1-\tau) Z_{j}+b$ where $\tau$ is the tax rate and $b$ is a constant shifter. Thus $Z_{a^{\prime}}$ is a known linear transformation of policy $Z_{j}$. We can construct $Z_{a^{\prime}}$ from $Z_{j}$. We can forecast under (A-2) using $\operatorname{Pr}\left(D_{j}=1 \mid Z_{j}=z\right)=\operatorname{Pr}\left(D_{a^{\prime}}=1 \mid Z_{a^{\prime}}=z\right)$. This assumes that the response to after tax wages is the same as the response to wages at the after tax level. The issue is whether $P_{a^{\prime} \mid X_{e}}$ lies in the historical support, or whether extrapolation is needed. Nonlinear versions of this example can be constructed.

As a final example, environmental economists use variation in one component of cost (e.g. travel cost) to estimate the effect of a new cost (e.g. a park registration fee). See Smith and Banzhaf (2004). Relating the costs and characteristics of new policies to the costs and characteristics of old policies is a standard, but sometimes controversial, method for forecasting the effects of new policies.

In the context of our model, extrapolation and forecasting are confined to constructing $P_{a^{\prime}}$ and its distribution. If policy $a^{\prime}$, characterized by vector $Z_{a^{\prime}}$, consists of new components that cannot be related to $Z_{j}, j \in \mathcal{H}_{e}$, or a base set of characteristics whose variation can be identified, the problem is intractable. Then $P_{a^{\prime}}$ and its distribution cannot be formed using econometric methods applied to historical data.

When it can be applied, our approach allows us to simplify the policy forecasting problem and concentrate our attention on forecasting choice probabilities and their distribution in solving the policy forecasting problem. We can use choice theory and choice data to construct these objects to forecast the impacts of new policies, by relating new policies to previously experienced policies.

\subsection{Forecasting the Effects of Policies in New Environments}

When the effects of policy $a$ are forecast for a new environment $e^{\prime}$ from baseline environment $e$, and $X_{e} \neq X_{e^{\prime}}$, in general both $\Delta^{M T E}\left(x, u_{D}\right)$ and $F_{P_{a} \mid X_{e}}$ will change. In general, neither object is environment invariant. $^{48}$ The new $X_{e^{\prime}}$ may have a different support than $X_{e}$ or any other environment in $\mathcal{H}$. In addition, the new $\left(X_{e^{\prime}}, U_{D}\right)$ stochastic relationship may be different from the historical $\left(X_{e}, U_{D}\right)$ stochastic relationship. Constructing $F_{P_{a} \mid X_{e^{\prime}}}$ from $F_{P_{a} \mid X_{e}}$ and $F_{Z_{a} \mid X_{e^{\prime}}}$ from $F_{Z_{a} \mid X_{e}}$ can be done using (i) functional form (including semiparametric functional restrictions) or (ii) analytic continuation methods. Notice that the maps $T_{a, j}$ and $\Phi_{a}$ may depend on $X_{e}$ and so the induced changes in these transformations must also

\footnotetext{
${ }^{48}$ We suppress the dependence of $U_{D}$ on $a$ for notational convenience.
} 
be modelled. There is a parallel discussion for $\Delta^{M T E}\left(x, u_{D}\right)$. The stochastic dependence between $X_{e^{\prime}}$ and $\left(U_{1}, U_{0}, U_{D}\right)$ may be different from the stochastic dependence between $X_{e}$ and $\left(U_{1}, U_{0}, U_{D}\right)$. We suppress the dependence of $U_{0}$ and $U_{1}$ on $e$ and $a$ only for convenience of exposition and make it explicit in the next paragraph.

Forecasting new stochastic relationships between $X_{e^{\prime}}$ and $\left(U_{1}, U_{0}, U_{D}\right)$ is a difficult task. It can be avoided if we invoke the traditional exogeneity assumptions of classical econometrics:

(A-9) $\left(U_{1, e, a}, U_{0, e, a}, U_{D, e, a}\right) \Perp\left(X_{e}, Z_{a}\right) \quad \forall e, a$.

Under (A-9), we only encounter the support problems for both $\Delta^{M T E}$ and the distribution of $\operatorname{Pr}\left(D_{a}=1 \mid Z_{a}, X_{e}\right)$ in constructing policy counterfactuals.

Conditions (A-7), (A-8) and (A-9) are unnecessary if the only goal of the analysis is to establish internal validity, the standard objective of the treatment effect literature. Autonomy and exogeneity conditions become important issues if we seek external validity. An important lesson from this analysis is that as we try to make the treatment effect literature do the tasks of structural econometrics (i.e. make out of sample forecasts), the assumptions invoked in the two literatures come together.

\subsection{A Comparison of Three Approaches}

Table V compares the strengths and limitations of the three approaches to policy evaluation that we have discussed: the structural approach, the conventional treatment effect approach, and the recently developed approach to treatment effects based on the MTE function developed in this paper.

The approach based on the MTE function and the structural approach share interpretability of parameters. Like the structural approach, it addresses a range of policy evaluation questions. The $M T E$ parameter is less comparable and less easily extrapolated across environments than are structural parameters, unless nonparametric versions of invariance and exogeneity assumptions are made. However, $\Delta^{M T E}$ is comparable across populations with different distributions of $P$ (conditional on $X_{e}$ ) and results from one population can be applied to another population under the conditions presented in this section. Analysts can use $\Delta^{M T E}$ to forecast a variety of policies. This invariance property is shared with conventional structural parameters. Our framework solves the problem of external validity which is ignored in the standard treatment effect approach. The price of these advantages of the structural approach is the greater range of 
econometric problems that must be solved. They are avoided in the conventional treatment approach at the cost of producing parameters that cannot be linked to well-posed economic models and hence do not provide building blocks for an empirically motivated general equilibrium analysis or for investigation of the impacts of new public policies. $\Delta^{M T E}$ estimates the preferences of the agents being studied and provides a basis for integration with well posed economic models. If the goal of a study is to examine one policy in place (the problem of internal validity), the stronger assumptions invoked in this section of the paper, and in structural econometrics, are unnecessary. Even if this is the only goal of the analysis, however, our approach allows the analyst to generate all treatment effects and $I V$ estimands from a common parameter and provides a basis for unification of the treatment effect literature.

\section{Monotonicity, Uniformity, Nonseparability, Independence and Policy Invariance: The Limits of Instrumental Variables}

The analysis of this paper and the entire recent literature on instrumental variables estimators for models with heterogeneous responses (i.e., models with outcomes of the form $(2 \mathrm{a})$ and $(2 \mathrm{~b})$ ) relies critically on the assumption that the treatment choice equation has a representation in the additively separable form (3). From Vytlacil (2002), we know that, under our assumptions. this assumption is equivalent to the assumption of monotonicity as defined by Imbens and Angrist (1994). Using the notation of Section 2, Imbens and Angrist define monotonicity as the following condition: if the $Z$ are changed for everyone from $Z=z$ to $Z=z^{\prime}, D_{z} \geq D_{z^{\prime}}$ or $D_{z} \leq D_{z^{\prime}}$ for all $U_{D}$ conditional on $X$. A better name for this condition would be "uniformity" since it describes a condition across people rather than the shape of a function for a particular person.

This uniformity condition imparts an asymmetry to the entire instrumental variable enterprise. Responses are permitted to be heterogeneous in a general way, but choices of treatment are not. In this section, we relax the assumption of additive separability in (3). We establish that in the absence of additive separability or uniformity, the entire instrumental variable identification strategy in this paper and the entire recent literature collapses. Parameters can be defined as weighted averages of an MTE but MTE and the derived parameters cannot be identified using any instrumental variable strategy. 
One natural benchmark nonseparable model is a random coefficient model of choice $D=\mathbf{1}[Z \beta \geq 0]$ where $\beta$ is a random coefficient vector and $\beta \Perp\left(Z, U_{0}, U_{1}\right)$. If $\beta$ is a random coefficient with a nondegenerate distribution and with components that take both positive and negative values, uniformity is clearly violated. However, it can be violated even when all components of $\beta$ are of the same sign if $Z$ is a vector.

To consider a more general case, relax the assumption of equation (3) to consider a more general case

$$
D^{*}=\mu_{D}\left(Z, U_{D}\right)
$$

where $\mu_{D}\left(Z, U_{D}\right)$ is not necessarily additively separable in $Z$ and $U_{D}$, and $U_{D}$ is not necessarily a scalar. ${ }^{49}$ In the random coefficient example, $U_{D}=\beta$.

$$
D=\mathbf{1}\left[D^{*} \geq 0\right]
$$

We maintain assumptions (A-1)-(A-5) and (A-8).

In special cases, (17a) can be expressed in an additively separable form. For example if $D^{*}$ is weakly separable in $Z$ and $U_{D}, D^{*}=\mu_{D}\left(\theta(Z), U_{D}\right)$ for any $U_{D}$ where $\theta(Z)$ is a scalar function, $\mu_{D}$ is increasing in $\theta(Z)$, and $U_{D}$ is a scalar, then we can write (17b) in the same form as (3):

$$
D=\mathbf{1}[\theta(Z) \geq \tilde{U}]
$$

where $\tilde{U}=\mu_{D}^{-1}\left(0 ; U_{D}\right)$ and $\tilde{U} \Perp Z \mid X$, and the inverse function is expressed with respect to the first argument. Vytlacil (2002) shows that any model that does not satisfy uniformity (or "monotonicity") will not have a representation in this form. ${ }^{50}$

In the additively separable case, the MTE (4) has three equivalent interpretations. (i) $U_{D}$ is the only unobservable in the first stage decision rule, and $M T E$ is the average effect of treatment given the unobserved characteristics in the decision rule $\left(U_{D}=u_{D}\right)$. (ii) A person with $U_{D}=u_{D}$ would be indifferent

\footnotetext{
${ }^{49}$ The additively separable latent index model is more general than it may at first appear. It is shown in Vytlacil (2004) that a wide class of threshold crossing models without the additive structure on the latent index will have a representation with the additively separable structure on the latent index.

${ }^{50}$ In the random coefficient case where $Z=\left(1, Z_{1}\right)$ where $Z_{1}$ is a scalar, and $\beta=\left(\beta_{0}, \beta_{1}\right)$ if $\beta_{1}>0$ for all realizations, we can write the choice rule in the form of (3): $Z_{1} \beta_{1}>-\beta_{0} \Rightarrow Z>-\frac{\beta_{0}}{\beta_{1}}$ and $U_{D}=-\frac{\beta_{0}}{\beta_{1}}$. This trick does not work in the general case.
} 
between treatment or not if $P(Z)=u_{D}$, where $P(Z)$ is a mean scale utility function. Thus, the $M T E$ is the average effect of treatment given that the individual would be indifferent between treatment or not if $P(Z)=u_{D}$. (iii) One can also view the additively separable form (3) as intrinsic in the way we are defining the parameter and interpret the $M T E(4)$ as an average effect conditional on the additive error term from the first stage choice model. Under all interpretations of the $M T E$ and under the assumptions used in the preceding sections of this paper, MTE can be identified by $L I V$; the MTE does not depend on $Z$ and hence it is policy invariant and the $M T E$ integrates up to generate all treatment effects, policy effects and all $I V$ estimands.

The three definitions are not the same in the general nonseparable case (17a). Heckman and Vytlacil (2001b) extend MTE in the nonseparable case using interpretation (i). MTE defined this way is policy invariant to changes in $Z$. They show that $L I V$ is a weighted average of the $M T E$ with possibly negative weights and does not identify MTE. If uniformity does not hold, the definition of MTE allows one to integrate MTE to obtain all of the treatment effects, but the instrumental variables estimator breaks down.

Alternatively, one could define $M T E$ based on (ii):

$$
\Delta_{B}^{M T E}(x, z)=E\left(Y_{1}-Y_{0} \mid X=x, U_{D} \in\left\{u_{D}: \mu_{D}\left(z, u_{D}\right)=0\right\}\right)
$$

This is the average treatment effect for individuals who would be indifferent between treatment or not at a given value of $z$. Heckman and Vytlacil (2001b) show that in the nonseparable case $L I V$ does not identify this $M T E$ and that MTE does not change when the distribution of $Z$ changes, provided the support of $M T E$ does not change. ${ }^{51}$ In general, this definition of MTE does not allow one to integrate up MTE to obtain the treatment parameters.

A third possibility is to force the index rule into an additive form by taking $\mu_{D}^{*}(Z)=E\left(\mu_{D}\left(Z, U_{D}\right) \mid Z\right)$, defining $U_{D}^{*}=\mu_{D}(Z, U)-E\left(\mu_{D}(Z, U) \mid Z\right)$ and define $M T E$ as $E\left(Y_{1}-Y_{0} \mid X=x, U_{D}^{*}=u_{D}^{*}\right)$. Note that $U_{D}^{*}$ is not independent of $Z$, is not policy invariant and is not structural. LIV does not estimate MTE. With this definition of the MTE it is not possible, in general, to integrate up MTE to obtain the various treatment effects.

\footnotetext{
${ }^{51}$ If the support of $Z$ changes, then the MTE must be extended to a new support.
} 
For any version of the nonseparable model, except those that can be transformed to separability, index sufficiency fails. To see this most directly, assume that $\mu_{D}\left(Z, U_{D}\right)$ is absolutely continuous with respect to Lebesgue measure. Define $\Omega(z)=\left\{u_{D}: \mu_{D}\left(z, u_{D}\right) \geq 0\right\}$. In the additively separable case, $P(z) \equiv \operatorname{Pr}(D=1 \mid Z=z)=\operatorname{Pr}\left(u_{D} \in \Omega(z)\right), P(z)=P\left(z^{\prime}\right) \Leftrightarrow \Omega(z)=\Omega\left(z^{\prime}\right)$. This produces index sufficiency. In the more general case of $(17 \mathrm{a})$ it is possible to have $\left(z, z^{\prime}\right)$ such that $P(z)=P\left(z^{\prime}\right)$ and $\Omega(z) \neq \Omega\left(z^{\prime}\right)$ so index sufficiency does not hold.

\subsection{Implications of Nonseparability}

In this section, we develop generalization (i), leaving development of the other interpretations for another occasion. We focus on PRTE. The analysis of the other treatment parameters follows by parallel arguments.

For any $u_{D}$ in the support of the distribution of $U_{D}$, define $\Omega_{u_{D}}=\left\{z: \mu_{D}\left(z, u_{D}\right) \geq 0\right\}$. For example, in the random coefficient case, with $U_{D} \equiv \beta$ and $D=\mathbf{1}[Z \beta \geq 0]$, we have $\Omega_{b}=\{z: z b \geq 0\}$, where $b$ is a realization of $\beta$. Define $\mathbf{1}_{\mathcal{A}}(t)$ to be the indicator function for the event $t \in \mathcal{A}$. Then, Appendix $\mathrm{B}$ shows that

$$
\begin{aligned}
E\left(Y_{a}\right)-E\left(Y_{a^{\prime}}\right) & =E\left[E\left(Y_{a} \mid X\right)-E\left(Y_{a^{\prime}} \mid X\right)\right] \\
& =\int\left[\int E\left(\Delta \mid X=x, U_{D}=u_{D}\right)\left(\begin{array}{c}
\operatorname{Pr}\left[Z_{a} \in \Omega_{u_{D}} \mid X=x\right] \\
-\operatorname{Pr}\left[Z_{a^{\prime}} \in \Omega_{u_{D}} \mid X=x\right]
\end{array}\right) d F_{U_{D} \mid X}\left(u_{D} \mid x\right)\right] d F_{X}(x)
\end{aligned}
$$

Thus, without additive separability, we can still derive an expression for PRTE and by similar reasoning the other treatment parameters. However, to evaluate the expression requires knowledge of MTE, of $\operatorname{Pr}\left[Z_{a} \in \Omega_{u_{D}} \mid X=x\right]$ and $\operatorname{Pr}\left[Z_{a^{\prime}} \in \Omega_{u_{D}} \mid X=x\right]$ for every $\left(u_{D}, x\right)$ in the support of the distribution of $\left(U_{D}, X\right)$, and of the distribution of $U_{D}$. In general, if no structure is placed on the $\mu_{D}$ function, one can normalize $U_{D}$ to be unit uniform (or a vector of unit uniform random variables) so that $F_{U_{D} \mid X}$ will be known. However, in this case the $\Omega_{u_{D}}=\left\{z: \mu_{D}\left(z, u_{D}\right) \geq 0\right\}$ sets will not in general be identified. If structure is placed on the $\mu_{D}$ function, one might be able to identify the $\Omega_{u_{D}}=\left\{z: \mu_{D}\left(z, u_{D}\right) \geq 0\right\}$ sets but then one needs to identify the distribution of $U_{D}$ conditional on $X$. If structure is placed on $\mu_{D}$, one cannot in general normalize the distribution of $U_{D}$ to be unit uniform without undoing the structure being imposed on $\mu_{D}$. 
In particular, consider the random coefficient model $D=\mathbf{1}[Z \beta \geq 0]$ where $U_{D}=\beta$ is a random vector, so that $\Omega_{\beta}=\{z: z \beta \geq 0\}$. In this case, if all of the other assumptions hold, including $Z \Perp \beta \mid X$, and the policy change does not affect $\left(Y_{1}, Y_{0}, X, \beta\right)$, the PRTE is given by

$$
\begin{aligned}
E\left(Y_{a}\right)-E\left(Y_{a^{\prime}}\right) & =E\left[E\left(Y_{a} \mid X\right)-E\left(Y_{a^{\prime}} \mid X\right)\right] \\
& =\int\left[\int E(\Delta \mid X=x, \beta=b)\left(\begin{array}{c}
\operatorname{Pr}\left[Z_{a} \in \Omega_{b} \mid X=x\right] \\
-\operatorname{Pr}\left[Z_{a^{\prime}} \in \Omega_{b} \mid X=x\right]
\end{array}\right) d F_{\beta \mid X}(b \mid x)\right] d F_{X}(x)
\end{aligned}
$$

Because structure has been placed on the $\mu_{D}(Z, \beta)$ function, the sets $\Omega_{\beta}$ are known. However, evaluating the function requires knowledge of the distribution of $\beta$ which will not in general be identified without further assumptions. ${ }^{52}$ Normalizing the distribution of $\beta$ to be a vector of unit uniform random variables produces the distribution of $\beta$ but eliminates the assumed linear index structure on $\mu_{D}$ and results in $\Omega_{\beta}$ sets that are not identified.

Even if the weights are identified, Heckman and Vytlacil (2001b) show that it is not possible to use $L I V$ to identify $M T E$ without additive separability between $Z$ and $U_{D}$ in the selection rule index. Appendix D develops this point for the random coefficient model. Thus, without additive separability in the latent index for the selection rule, we can still create an expression for PRTE (and the other treatment parameters) but both the weights and the MTE function are no longer identified using instrumental variables.

One superficially plausible way to avoid these problems would be to define $\tilde{\mu}_{D}(Z)=E\left(\mu_{D}\left(Z, U_{D}\right) \mid Z\right)$ and $\tilde{U}_{D}=\mu_{D}\left(Z, U_{D}\right)-E\left(\mu_{D}\left(Z, U_{D}\right) \mid Z\right)$, producing the model $D=\mathbf{1}\left[\tilde{\mu}_{D}(Z)-\tilde{U}_{D} \geq 0\right]$. We keep the conditioning on $X$ implicit. One could redefine $M T E$ using $\tilde{U}_{D}$ and proceed as if the true model possessed additive separability between observables and unobservables in the latent index. This is the method pursued in approach (iii).

For two reasons, this approach does not solve the problem of providing an adequate generalization of MTE. First, with this definition, $\tilde{U}_{D}$ is a function of $\left(Z, U_{D}\right)$, and a policy that changes $Z$ will then also change $\tilde{U}_{D}$. Thus, policy invariance of the MTE no longer holds. Second, this approach generates a $\tilde{U}_{D}$ that is no longer statistically independent of $Z$ so that assumption (A-2) no longer holds when $\tilde{U}_{D}$ is substituted for $U_{D}$ even when (A-2) is true for $U_{D}$. Lack of independence between observables and

\footnotetext{
${ }^{52}$ See, e.g. Ichimura and Thompson (1998) for conditions for identifying the distribution of $\beta$ in a random coefficient discrete choice model when $Z \Perp \beta$.
} 
unobservables in the latent index both invalidates our expression for PRTE (and the expressions for the other treatment effects) and causes $L I V$ to no longer identify $M T E$.

The nonseparable model can also restrict the support of $P(Z)$. For example, consider a standard normal random coefficient model with a scalar regressor $\left(Z=\left(1, Z_{1}\right)\right)$. Assume $\beta_{0} \sim N\left(0, \sigma_{0}^{2}\right), \beta_{1} \sim N\left(\bar{\beta}_{1}, \sigma_{1}^{2}\right)$, and $\beta_{0} \Perp \beta_{1}$. Then

$$
P\left(z_{1}\right)=\Phi\left(\frac{\bar{\beta}_{1} z_{1}}{\sqrt{\sigma_{0}^{2}+\sigma_{1}^{2} z_{1}^{2}}}\right),
$$

where in this usage $\Phi$ is the standard cumulative normal distribution. If the support of $z_{1}$ is $\Re^{1}$, then in the standard additive model $\sigma_{1}^{2}=0$ and $P\left(z_{1}\right)$ has support $[0,1]$. When $\sigma_{1}^{2}>0$, the support is strictly within the unit interval. ${ }^{53}$ In the special case when $\sigma_{0}^{2}=0$, the support is one point $\left(P(z)=\Phi\left(\frac{\bar{\beta}_{1}}{\sigma_{1}}\right)\right)$. We cannot, in general, identify ATE, TT or any treatment effect requiring the endpoints 0 or 1.

Thus the more general case model of nonuniformity presented in this section does not satisfy the index sufficiency property, and the support of the treatment effects and estimators is, in general, less than full. The random coefficient model for choice may explain the empirical support problems for $P(Z)$ found in Heckman, Ichimura, Smith, and Todd (1998).

\subsection{Implications of Dependence}

We next consider relaxing the independence assumption (A-2) to allow $Z \not K U_{D} \mid X$ while maintaining the assumption that $Z \Perp\left(Y_{1}, Y_{0}\right) \mid\left(X, U_{D}\right)$. We maintain the other assumptions, including additive separability between $Z$ and $U_{D}$ in the latent index for the selection rule (equation (3)) and the assumption that the policy changes $Z$ but does not change $\left(U_{D}, Y_{0}, Y_{1}, X\right)$. Thus we assume that the policy change does not change the MTE function (policy invariance). Given these assumptions, we derive in Appendix B the following expression for PRTE in the nonindependent case:

$$
\begin{aligned}
& E\left(Y_{a}\right)-E\left(Y_{a^{\prime}}\right)=E\left[E\left(Y_{a} \mid X\right)-E\left(Y_{a^{\prime}} \mid X\right)\right] \\
& =\int\left[\int E\left(\Delta \mid X=x, U_{D}=u_{D}\right)\left(\begin{array}{c}
\operatorname{Pr}\left[\mu_{D}\left(Z_{a^{\prime}}\right)<u_{D} \mid X=x, U_{D}=u_{D}\right] \\
-\operatorname{Pr}\left[\mu_{D}\left(Z_{a}\right)<u_{D} \mid X=x, U_{D}=u_{D}\right]
\end{array}\right) d F_{U_{D} \mid X}\left(u_{D} \mid x\right)\right] d F_{X}(x) .
\end{aligned}
$$

\footnotetext{
${ }^{53}$ The interval is $\left[\Phi\left(\frac{-\left|\beta_{1}\right|}{\sigma_{1}}\right), \Phi\left(\frac{\left|\beta_{1}\right|}{\sigma_{1}}\right)\right]$.
} 
Although we can derive an expression for PRTE without requiring independence between $Z$ and $U_{D}$, to evaluate this expression requires knowledge of $M T E$ and of $\operatorname{Pr}\left[\mu_{D}\left(Z_{a^{\prime}}\right)<u_{D} \mid X=x, U_{D}=u_{D}\right]$ and of $\left.\operatorname{Pr}\left[\mu_{D}\left(Z_{a}\right)<u_{D} \mid X=x, U_{D}=u_{D}\right]\right)$ for every $\left(x, u_{D}\right)$ in the support of the distribution of $\left(X, U_{D}\right)$. This requirement is stronger than in the case of independence since the weights no longer depend only on the distribution of $P_{a}\left(Z_{a}\right)$ and $P_{a^{\prime}}\left(Z_{a^{\prime}}\right)$ conditional on $X$. To evaluate these weights requires knowledge of the function $\mu_{D}$ and of the joint distribution of $\left(U_{D}, Z_{a}\right)$ and $\left(U_{D}, Z_{a^{\prime}}\right)$ conditional on $X$, and these will in general not be identified without further assumptions.

Even if the weights are identified. Heckman and Vytlacil (2001b) show that it is not possible to use $L I V$ to identify $M T E$ without independence between $Z$ and $U_{D}$ conditional on $X$. Thus, without conditional independence between $Z$ and $U_{D}$ in the latent index for the decision rule, we can still create an expression for PRTE but both the weights and the MTE function are no longer identified without invoking further assumptions.

One superficially appealing way to avoid these problems is to define $\tilde{U}_{D}=F_{U_{D} \mid X, Z}\left(U_{D}\right)$ and $\tilde{\mu}_{D}(Z)=$ $F_{U_{D} \mid X, Z}\left(\mu_{D}(Z)\right)$, so $D=\mathbf{1}\left[\mu_{D}(Z)-U_{D} \geq 0\right]=\mathbf{1}\left[\tilde{\mu}_{D}(Z)-\tilde{U}_{D} \geq 0\right]$ with $\tilde{U}_{D} \sim U n i f[0,1]$ conditional on $X$ and $Z$ and so $\tilde{U}_{D}$ is independent of $X$ and $Z$. It might seem that the previous analysis would carry over. However, by defining $\tilde{U}_{D}=F_{U_{D} \mid X, Z}\left(U_{D}\right)$, we have defined $\tilde{U}_{D}$ in a way that depends functionally on $Z$ and $X$, and hence we violate invariance of the $M T E$ with respect to the shifts in the distribution of $Z$ given $X$.

\subsection{Do We Need Uniformity?}

The monotonicity or uniformity condition and the additional condition of positive weights for $M T E$ are both required to obtain gross treatment effects using $I V$. If these conditions are violated, changes in $Z$ induce two way flows with some people changing into treatment and others leaving it. Thus $I V$ does not identify the "gross effect" of treatment. Recall from our discussion in Section 4.3 that even if we have monotonicity or uniformity as defined in this section (a necessary and sufficient condition for the existence of representation (4)), the discussion in Section 4.3 reveals that in a model with multiple instruments we may still obtain negative $I V$ weights unless we condition on the other instruments.

Monotonicity and independence are invoked when the treatment (indicated by $D$ ) is the policy being evaluated. But treatments are only a subset of all possible policies of interest and if the goal is to evaluate 
the effects of a policy on aggregate outcomes, as in $\Delta^{P R T E}$, the monotonicity requirement may not be needed. In that case, one is interested in the net impact of the policy and not the impact of treatment operating through a particular mechanism or treatment. The policy of interest may entail two-way flows.

Consider the case where $D$ indicates schooling, which is the treatment. Define $D=1$ if the person goes to college and $D=0$ otherwise. Suppose that the policy being studied is the introduction of a physical education (PE) requirement in colleges along with mandatory augmented athletics facilities. The policy has no effect on $\left(Y_{1}, Y_{0}\right)$ (e.g. potential earnings) but it affects the choice of college, so it is a valid $Z$. Some people hate PE while others love it and are attracted by colleges with good gyms, so monotonicity (uniformity) is violated. If $Z_{a}=z$ is the policy with $\mathrm{PE}$ and $Z_{a^{\prime}}=z^{\prime}$ is the policy without PE, $E\left(Y \mid Z_{a}=z\right)-E\left(Y \mid Z_{a^{\prime}}=z^{\prime}\right)$ is a perfectly valid policy parameter - the effect of the policy on aggregate outcomes - even if uniformity is violated and $\Delta^{M T E}$ is not policy invariant. One only needs uniformity, policy invariance and the other assumptions only when the policy is a treatment.

\subsection{The Limits of Instrumental Variable Estimators}

The treatment effect literature focuses on a class of policies that move treatment choices in the same direction for everyone. General instruments do not have universally positive weights on $\Delta^{M T E}$. They are not guaranteed to shift everyone in the same direction. They do not necessarily estimate gross treatment effects. However, the effect of treatment is not always the parameter of policy interest. Thus, in the example just presented, schooling is the vehicle through which policy operates. One might be interested in the effect of schooling (the treatment effect) or the effect of the policy. These are separate issues unless the policy is the treatment.

Generalizing the MTE to the case of a nonseparable choice equation that violates the monotonicity condition, we can define but cannot identify the policy parameters of interest. If we make the model symmetrically heterogeneous in outcome and choice equations, the method of instrumental variables and our extensions of it break down in terms of estimating economically interpretable parameters. This case is beyond the outer limits of an entire literature, although it captures intuitively plausible phenomena. More general structural methods are required. ${ }^{54}$

\footnotetext{
${ }^{54}$ The framework of Carneiro, Hansen, and Heckman (2003) can be generalized to allow for random coefficients models in choice equations, and lack of policy invariance in the sense of (A-7). However, a fully semiparametric analysis does not
} 


\section{Summary and Proposed Extensions}

This paper develops an approach to policy evaluation based on the marginal treatment effect $\left(\Delta^{M T E}\right)$, which provides a choice-theoretic foundation for organizing the treatment effect literature. All of the conventional treatment effect parameters can be expressed as different weighted averages of $\Delta^{M T E}$. These conventional treatment effect parameters do not, in general, answer economically interesting questions. We define the policy relevant treatment effect as the solution to a Benthamite policy criterion for policies operating on decisions to participate, but not on potential outcomes. The policy relevant treatment effect can be represented as a weighted average of $\Delta^{M T E}$ where the weights differ in general from the weights used to generate conventional treatment effects. Thus the conventional treatment effects are not guaranteed to answer policy relevant questions.

Instrumental variable estimators and $O L S$ estimators converge to expressions that can be represented as weighted averages of $\Delta^{M T E}$ parameters, with the weights in general different from those used to define the various treatment effects and the weights not necessarily positive so they do not identify a gross treatment effect. We show how to check whether the weights are positive. Conventional $I V$ and matching assumptions impose a strong condition on the $\Delta^{M T E}$ _ that selection into programs is not made in terms of any unobservable gain from program participation.

We present methods for estimating $\Delta^{M T E}$ based on local instrumental variables and we develop a new instrumental variable for recovering policy relevant treatment effects using standard instrumental variable methods. We present conditions for using $I V$ to estimate well-posed policy questions. We show that $I V$ need not generate any interesting policy counterfactual, and that there are policy counterfactuals for which no $I V$ can be generated. In a model of heterogeneous responses, there is no natural superiority of conventional $I V$ over $O L S$ in estimating policy relevant parameters. We develop the conditions required to forecast the effects of old policies on new environments and the effects of new policies. These issues are typically ignored in the treatment effect literature but are central to the structural policy evaluation literature.

The model presented in this paper and the models presented in the recent literature on instrumental variables in heterogeneous response models are fundamentally asymmetric. Responses to treatment are appear to be possible. This generalization is under preparation for publication. 
allowed to be heterogeneous in a general way, but choices of treatment are not. When we develop a symmetrically heterogeneous model, the method of instrumental variables breaks down entirely, and a different approach to econometric policy analysis is required.

Department of Economics, University of Chicago, 1126 E. 59th Street, Chicago, IL, 60637, U.S.A. jjh@uchicago.edu,

and

Dept. of Economics, Stanford University, 579 Serra Mall, Palo Alto, CA, 94305, U.S.A. vytlacil@stanford.edu

\section{Appendices}

\section{A Testable Monotonicity in $P$ Restriction}

Theorem 1. Assume $Y_{0}, Y_{1}$, and $D$ are determined by equations 2a, $2 b$ and 3 respectively. Assume conditions (A-1) through $(A-5)$ hold.

(i) Let $g_{0}, g_{1}$ be any real valued functions such that $g_{0}\left(Y_{0}, X\right), g_{1}\left(Y_{1}, X\right) \geq 0$ with probability 1 . Then $E\left((1-D) g_{0}(Y, X) \mid X, P(Z)=p\right)$ is weakly decreasing in $p$ and $E\left(D g_{1}(Y, X) \mid X, P(Z)=p\right)$ is weakly increasing in $p$.

(ii) Let $g_{0}, g_{1}$ be any real valued functions such that $g_{0}\left(Y_{0}, X\right), g_{1}\left(Y_{1}, X\right)>0$ with probability 1 . Then $E\left((1-D) g_{0}(Y, X) \mid X, P(Z)=p\right)$ is strictly decreasing in $p$ and $E\left(D g_{1}(Y, X) \mid X, P(Z)=p\right)$ is strictly increasing in $p$.

Proof. Consider assertion (i). Consider $E\left(D g_{1}(Y, X) \mid X=x, P(Z)=p\right)$ for some $x$. Let $p_{1}$, $p_{0}$ denote any two points in the support of the distribution of $P(Z)$ conditional on $X=x$ such that $p_{1}>p_{0}$. Then 


$$
\begin{aligned}
E(D & \left.g_{1}(Y, X) \mid X=x, P(Z)=p_{1}\right)-E\left(D g_{1}(Y, X) \mid X=x, P(Z)=p_{0}\right) \\
& =E\left(\mathbf{1}\left[U_{D} \leq P(Z)\right] g_{1}\left(Y_{1}, X\right) \mid X=x, P(Z)=p_{1}\right)-E\left(\mathbf{1}\left[U_{D} \leq P(Z)\right] g_{1}\left(Y_{1}, X\right) \mid X=x, P(Z)=p_{0}\right) \\
& =E\left(\mathbf{1}\left[U_{D} \leq p_{1}\right] g_{1}\left(Y_{1}, X\right) \mid X=x\right)-E\left(\mathbf{1}\left[U_{D} \leq p_{0}\right] g_{1}\left(Y_{1}, X\right) \mid X=x\right) \\
& =E\left(\left\{\mathbf{1}\left[U_{D} \leq p_{0}\right]+\mathbf{1}\left[p_{0}<U_{D} \leq p_{1}\right]\right\} g_{1}\left(Y_{1}, X\right) \mid X=x\right)-E\left(\mathbf{1}\left[U_{D} \leq p_{0}\right] g_{1}\left(Y_{1}, X\right) \mid X=x\right) \\
& =E\left(\mathbf{1}\left[p_{0}<U_{D} \leq p_{1}\right] g_{1}\left(Y_{1}, X\right) \mid X=x\right) \\
& \geq 0
\end{aligned}
$$

where the first equality follows from the definition of $D$ and uses the fact that $D g_{1}(Y, X)=D g_{1}\left(Y_{1}, X\right)$; the second equality uses independence condition (A-2); the third equality uses the fact that $p_{0}<p_{1}$ and thus that $\mathbf{1}\left[U_{D} \leq p_{1}\right]=\mathbf{1}\left[U_{D} \leq p_{0}\right]+\mathbf{1}\left[p_{0}<U_{D} \leq p_{1}\right]$; the fourth equality follows from linearity of expectations; the final inequality follows from $g_{1}\left(Y_{1}, X\right) \geq 0$ with probability 1 . The proof that $E\left((1-D) g_{0}(Y, X) \mid\right.$ $X, P(Z)=p)$ is decreasing in $p$ follows from a similar argument. Assertion (ii) follows from a trivial modification of the last line of the above proof.

Consider the following examples of $g_{0}$ and $g_{1}$ :

(i) If $Y_{1}, Y_{0}$ are known to be nonnegative (for example, $Y_{1}, Y_{0}$ are indicator variables, or $Y_{1}, Y_{0}$ are wages), then we may choose $g_{j}(Y, X)=Y$. In this example, Theorem 1 implies that $E((1-D) Y \mid X, P(Z)=$ $p)$ is weakly decreasing in $p$ and $E(D Y \mid X, P(Z)=p)$ is weakly increasing in $p$. More generally, if $Y_{1}, Y_{0}$ are known to be bounded from below by a function of $X$ so that $Y_{1} \geq l_{1}(X), Y_{0} \geq l_{0}(X)$ with probability 1 , then we may choose $g_{j}(Y, X)=Y-l_{j}(X)$ so that Theorem 1 implies that $E\left((1-D)\left(Y-l_{0}(X)\right) \mid X, P(Z)=p\right)$ is weakly decreasing in $p$ and $E\left(D\left(Y-l_{1}(X)\right) \mid X, P(Z)=p\right)$ is weakly increasing in $p$.

(ii) Without any assumptions on the support of the distribution of $Y_{1}, Y_{0}$, let $t$ denote a real number and take $g_{j}(Y, X)=\mathbf{1}[Y \leq t]$ for $j=0,1$. Then Theorem 1 implies that $\operatorname{Pr}(D=0, Y \leq t \mid X, P(Z)=p)$ is weakly decreasing in $p$ and $\operatorname{Pr}(D=1, Y \leq t \mid X, P(Z)=p)$ is weakly increasing in $p$. More generally, let $\mathcal{A}$ denote any measurable subset of the real line, and take $g_{j}(Y, X)=\mathbf{1}[Y \in \mathcal{A}]$ for 
$j=0,1$. Then the conclusion of the proposition can be rewritten as $\operatorname{Pr}(D=0, Y \in \mathcal{A} \mid X, P(Z)=p)$ is weakly decreasing in $p$ and $\operatorname{Pr}(D=1, Y \in \mathcal{A} \mid X, P(Z)=p)$ is weakly increasing in $p$.

For any choice of $g_{0}, g_{1}$, the restriction of Theorem 1 leads to the prediction that regression functions $E\left((1-D) g_{0}(Y, X) \mid X, P(Z)=p\right)$ and $E\left(D g_{1}(Y, X) \mid X, P(Z)=p\right)$ satisfy the monotonicity conditions. This is an example of a nonparametric null with shape restrictions versus a nonparametric alternative. A formal test of the null hypothesis can be implemented using the methodology of Ghosal, Sen, and van der Vaart (2000).

The restrictions of Theorem 1 nest the Imbens-Rubin (1997) restrictions on $I V$ as a special case. They assume a binary $Z$, and obtain the density of $Y_{1}$ and $Y_{0}$ from the observed data and derive the testable restriction that these densities be nonnegative. ${ }^{55}$ Our analysis is more general. ${ }^{56}$

\section{B Derivation of $P R T E$ and $I V$ Weights}

Proof. (Equation (6)) To simplify the notation, assume that $V(Y)=Y$. Modifications required for the more general case are obvious. Define $\mathbf{1}_{\mathcal{A}}(t)$ to be the indicator function for the event $t \in \mathcal{A}$. Then

$E\left(Y_{a} \mid X\right)$

$$
\begin{aligned}
& =\int_{0}^{1} E\left(Y_{a} \mid X, P_{a}\left(Z_{a}\right)=p\right) d F_{P_{a} \mid X}(p) \\
& =\int_{0}^{1}\left[\int_{0}^{1}\left[\mathbf{1}_{[0, p]}\left(u_{D}\right) E\left(Y_{1, a} \mid X, U_{D}=u_{D}\right)+\mathbf{1}_{(p, 1]}\left(u_{D}\right) E\left(Y_{0, a} \mid X, U_{D}=u_{D}\right) d u\right] d F_{P_{a} \mid X}(p)\right. \\
& =\int_{0}^{1}\left[\int_{0}^{1}\left[\mathbf{1}_{\left[u_{D}, 1\right]}(p) E\left(Y_{1, a} \mid X, U_{D}=u_{D}\right)+\mathbf{1}_{\left(0, u_{D}\right]}(p) E\left(Y_{0, a} \mid X, U_{D}=u_{D}\right) d F_{P_{a} \mid X}(p)\right] d u_{D}\right. \\
& =\int_{0}^{1}\left[\left(1-F_{P_{a} \mid X}\left(u_{D}\right)\right) E\left(Y_{1, a} \mid X, U_{D}=u_{D}\right)+F_{P_{a} \mid X}\left(u_{D}\right) E\left(Y_{0, a} \mid X, U_{D}=u_{D}\right)\right] d u_{D} .
\end{aligned}
$$

\footnotetext{
${ }^{55}$ See also Heckman, Smith, and Taber (1998) for a closely related test.

${ }^{56}$ For ease of exposition, suppress conditioning on $X$. Take the case where $Z=0,1$, and $P(1)>P(0)$. Consider the $Y_{1}$ outcome; the analysis for $Y_{0}$ is completely symmetric. For binary $Z$ with $P(1)>P(0)$, our restriction can be rewritten as $E\left(D g_{1}(Y) \mid Z=1\right) \geq E\left(D g_{1}(Y) \mid Z=0\right)$. Take $g_{1}(Y)=\mathbf{1}[Y \in \mathcal{A}]$ for any pre-specified set $\mathcal{A}$, for example, the intervals examined in the histogram analyzed in the Imbens-Rubin paper. In this special case, our monotonicity restriction is that $\operatorname{Pr}(D=1, Y \in \mathcal{A} \mid Z=1)-\operatorname{Pr}(D=0, Y \in \mathcal{A} \mid Z=0)>0$, and the restriction is the same as the Imbens and Rubin restriction of a nonnegative density. Our analysis replaces their densities with the probability that $Y$ lies in any given set. Thus, their restriction is a very special case of the general monotonicity restriction developed in this paper.
} 
This derivation involves changing the order of integration. Note that from (A-4),

$$
E\left|\mathbf{1}_{[0, p]}\left(u_{D}\right) E\left(Y_{1, a} \mid X, U_{D}=u_{D}\right)+\mathbf{1}_{(p, 1]}\left(u_{D}\right) E\left(Y_{0, a} \mid X, U_{D}=u_{D}\right)\right| \leq E\left(\left|Y_{1}\right|+\left|Y_{0}\right|\right)<\infty
$$

so the change in the order of integration is valid by Fubini's theorem. Comparing policy $a$ to policy $a^{\prime}$,

$$
E\left(Y_{a} \mid X\right)-E\left(Y_{a^{\prime}} \mid X\right)=\int_{0}^{1} E\left(\Delta \mid X, U_{D}=u_{D}\right)\left(F_{P_{a^{\prime}} \mid X}\left(u_{D}\right)-F_{P_{a} \mid X}\left(u_{D}\right)\right) d u_{D}
$$

which gives the required weights. (Recall $\Delta=Y_{1}-Y_{0}$ and from (A-7) we can drop the $a, a^{\prime}$ subscripts on outcomes and errors.)

Q.E.D.

\section{Relaxing (A-7): Implications of Noninvariance for PRTE.}

Suppose that all of the assumptions invoked up to Section 3 are satisfied, including additive separability in the latent index choice equation (3) (equivalently, the monotonicity or uniformity condition). Impose the normalization that the distribution of $U_{D}$ is unit uniform. Suppose however, contrary to (A-7), that the distribution of $\left(Y_{1}, Y_{0}, U_{D}, X\right)$ is different under the two regimes $a$ and $a^{\prime}$. Thus, let $\left(Y_{1, a}, Y_{0, a}, U_{D, a}, X_{a}\right)$ and $\left(Y_{1, a^{\prime}}, Y_{0, a^{\prime}}, U_{D, a^{\prime}}, X_{a^{\prime}}\right)$ denote the random vectors under regimes $a$ and $a^{\prime}$, respectively. Following the same analysis as used to derive equation (6), the PRTE conditional on $X$ is given by

$$
\begin{aligned}
E\left(Y_{a} \mid\right. & \left.X_{a}=x\right)-E\left(Y_{a^{\prime}} \mid X_{a^{\prime}}=x\right) \\
& =\int_{0}^{1} E\left(Y_{1, a}-Y_{0, a} \mid X_{a}=x, U_{D, a^{\prime}}=u\right)\left[F_{P_{a^{\prime}} \mid X_{a^{\prime}}}(u \mid x)-F_{P_{a} \mid X_{a}}(u \mid x)\right] d u \\
& +\int_{0}^{1}\left[E\left(Y_{0, a} \mid X_{a}=x, U_{D, a}=u\right)-E\left(Y_{0, a^{\prime}} \mid X_{a^{\prime}}=x, U_{D, a^{\prime}}=u\right)\right] d u \\
& +\int_{0}^{1}\left[\left(1-F_{P_{a^{\prime}} \mid X}(u \mid x)\right)\left(E\left(Y_{1, a}-Y_{0, a} \mid X_{a}=x, U_{D, a}=u\right)-E\left(Y_{1, a^{\prime}}-Y_{0, a^{\prime}} \mid X_{a^{\prime}}, U_{D, a^{\prime}}=u\right)\right)\right] d u .
\end{aligned}
$$

Thus, when the policy affects the distribution of $\left(Y_{1}, Y_{0}, U_{D}, X\right)$, the PRTE is given by the sum of three terms: (I) the value of PRTE if the policy did not affect $\left(Y_{1}, Y_{0}, X, U_{D}\right)$; (II) the weighted effect of the policy change on $E\left(Y_{0} \mid X, U_{D}\right)$; and (III) the weighted effect of the policy change on MTE. Evaluating the PRTE requires knowledge of the MTE function in both regimes, knowledge of $E\left(Y_{0} \mid X=x, U_{D}=u\right)$ in both regimes, as well as knowledge of the distribution of $P(Z)$ in both regimes. Note, however, that 
if we assume that the distribution of $\left(Y_{1, a}, Y_{0, a}, U_{D, a}\right)$ conditional on $X_{a}=x$ equals the distribution of $\left(Y_{1, a^{\prime}}, Y_{0, a^{\prime}}, U_{D, a^{\prime}}\right)$ conditional on $X_{a^{\prime}}=x$, then $E\left(Y_{1, a} \mid U_{D, a}=u, X_{a}=x\right)=E\left(Y_{1, a^{\prime}} \mid U_{D, a^{\prime}}=u, X_{a^{\prime}}=x\right)$, $E\left(Y_{0, a} \mid U_{D, a}=u, X_{a}=x\right)=E\left(Y_{0, a^{\prime}} \mid U_{D, a^{\prime}}=u, X_{a^{\prime}}=x\right)$, and thus that terms (II) and (III) are zero and the expression for PRTE simplifies to the expression of equation (6).

\section{Proof. (Equation (10))}

$$
\begin{aligned}
\operatorname{Cov}\left(J_{x}(Z), Y \mid X\right) & =E\left(\left[J_{x}(Z)-E(J(Z) \mid X)\right] Y \mid X\right) \\
& =E\left((J(Z)-E(J(Z) \mid X))\left(Y_{0}+D\left(Y_{1}-Y_{0}\right)\right) \mid X\right) \\
& =E\left((J(Z)-E(J(Z) \mid X)) D\left(Y_{1}-Y_{0}\right) \mid X\right) \\
& =E\left(\tilde{J}(Z) \mathbf{1}\left[U_{D} \leq P(Z)\right]\left(Y_{1}-Y_{0}\right) \mid X\right) \\
& =E\left(\tilde{J}(Z) \mathbf{1}\left[U_{D} \leq P(Z)\right] E\left(Y_{1}-Y_{0} \mid X, Z, U_{D}\right) \mid X\right) \\
& =E\left(\tilde{J}(Z) \mathbf{1}\left[U_{D} \leq P(Z)\right] E\left(Y_{1}-Y_{0} \mid X, U_{D}\right) \mid X\right) \\
& =E\left(E\left[\tilde{J}(Z) \mathbf{1}\left[U_{D} \leq P(Z)\right] \mid X=x, U_{D}\right] E\left(Y_{1}-Y_{0} \mid X, U_{D}\right) \mid X\right) \\
& =\int_{0}^{1}\left\{E\left(\tilde{J}(Z) \mid X, P(Z) \geq u_{D}\right) \operatorname{Pr}\left(P(Z) \geq u_{D}\right) E\left(Y_{1}-Y_{0} \mid X, U_{D}=u_{D}\right)\right\} d u_{D} \\
& =\int_{0}^{1} \Delta^{M T E}\left(X, u_{D}\right) E\left(\tilde{J}(Z) \mid X, P(Z) \geq u_{D}\right) \operatorname{Pr}\left(P(Z) \geq u_{D}\right) d u_{D}
\end{aligned}
$$

The third equality follows from (3); the fourth equality follows from the law of iterated expectations with the inside expectation conditional on $\left(X, Z, U_{D}\right)$; the fifth equality follows from assumption (A-2); the sixth equality follows from the law of iterated expectations with the inside expectation conditional on $\left(X=x, U_{D}\right)$; the seventh equality follows from Fubini's Theorem and the normalization that $U_{D}$ is distributed unit uniform conditional on $X$; and the final equality follows from plugging in the definition of $\Delta^{M T E}$. Yitzhaki $(1996,1999)$ was the first to develop the interpretation of $I V$ as a weighted average although he did not develop the MTE.

Q.E.D. 
Proof. (Equation (18)) E ( $\left.Y_{a} \mid X\right)$

$$
\begin{aligned}
& =\int E\left(Y_{a} \mid X, U_{D}=u_{D}, Z_{a}=z\right) d F_{U_{D}, Z_{a} \mid X}\left(u_{D}, z\right) \\
& =\int\left(\mathbf{1}_{\Omega_{u_{D}}}(z) E\left(Y_{1} \mid X, U_{D}=u_{D}, Z_{a}=z\right)+\mathbf{1}_{\Omega_{u_{D}}^{c}}(z) E\left(Y_{0} \mid X, U_{D}=u_{D}, Z_{a}=z\right)\right) d F_{U_{D}, Z_{a} \mid X}\left(u_{D}, z\right) \\
& =\int\left(\mathbf{1}_{\Omega_{u_{D}}}(z) E\left(Y_{1} \mid X, U_{D}=u_{D}\right)+\mathbf{1}_{\Omega_{u_{D}}^{c}}(z) E\left(Y_{0} \mid X, U_{D}=u_{D}\right)\right) d F_{U_{D}, Z_{a} \mid X}\left(u_{D}, z\right) \\
& =\int\left[\int\left(\mathbf{1}_{\Omega_{u_{D}}}(z) E\left(Y_{1} \mid X, U_{D}=u_{D}\right)+\mathbf{1}_{\Omega_{u_{D}}^{c}}(z) E\left(Y_{0} \mid X, U_{D}=u_{D}\right)\right) d F_{Z_{a} \mid X}(z)\right] d F_{U_{D} \mid X}\left(u_{D}\right) \\
& =\int\left[\operatorname{Pr}\left[Z_{a} \in \Omega_{u_{D}} \mid X\right] E\left(Y_{1} \mid X, U_{D}=u_{D}\right)+\left(1-\operatorname{Pr}\left[Z_{a} \in \Omega_{u_{D}} \mid X\right]\right) E\left(Y_{0} \mid X, U_{D}=u_{D}\right)\right] d F_{U_{D} \mid X}\left(u_{D}\right)
\end{aligned}
$$

where $\Omega_{u_{D}}^{c}$ denotes the complement of $\Omega_{u_{D}}$ and where the first equality follows from the law of iterated expectations; the second equality follows by plugging in our threshold crossing model for $D$; the third equality follows from independence $Z \Perp\left(Y_{1}, Y_{0}, U_{D}\right) \mid X$; the fourth and fifth equalities follow by an application of Fubini's Theorem and a rearrangement of terms. Fubini's Theorem may be applied by assumption (A-4). Thus comparing policy $a$ to policy $a^{\prime}$, we obtain (18).

$$
E\left(Y_{a} \mid X\right)-E\left(Y_{a^{\prime}} \mid X\right)=\int E\left(\Delta \mid X, U_{D}=u_{D}\right)\left(\operatorname{Pr}\left[Z_{a} \in \Omega_{u_{D}} \mid X\right]-\operatorname{Pr}\left[Z_{a^{\prime}} \in \Omega_{u_{D}} \mid X\right]\right) d F_{U_{D} \mid X}\left(u_{D}\right)
$$

Q.E.D.

Proof. (Equation (19)) 
$E\left(Y_{a} \mid X\right)$

$$
\begin{aligned}
& =\int E\left(Y_{a} \mid X, U_{D}=u_{D}, Z_{a}=z\right) d F_{U_{D}, Z_{a} \mid X}\left(u_{D}, z\right) \\
& =\int\left[\begin{array}{c}
\mathbf{1}_{\left[0, \mu_{D}(z)\right]}\left(u_{D}\right) E\left(Y_{1} \mid X, Z=z, U_{D}=u_{D}\right) \\
+\mathbf{1}_{\left(\mu_{D}(z), 1\right]}\left(u_{D}\right) E\left(Y_{0} \mid X, Z=z, U_{D}=u_{D}\right)
\end{array}\right] d F_{U_{D}, Z_{a} \mid X}\left(u_{D}, z\right) \\
& =\int\left[\begin{array}{c}
\mathbf{1}_{\left[0, \mu_{D}(z)\right]}\left(u_{D}\right) E\left(Y_{1} \mid X, U_{D}=u_{D}\right) \\
+\mathbf{1}_{\left(\mu_{D}(z), 1\right]}\left(u_{D}\right) E\left(Y_{0} \mid X, U_{D}=u_{D}\right)
\end{array}\right] d F_{U_{D}, Z_{a} \mid X}\left(u_{D}, z\right) \\
& =\int\left[\int\left(\begin{array}{c}
\mathbf{1}_{\left[0, \mu_{D}(z)\right]}\left(u_{D}\right) E\left(Y_{1} \mid X, U_{D}=u_{D}\right) \\
+\mathbf{1}_{\left(\mu_{D}(z), 1\right]}\left(u_{D}\right) E\left(Y_{0} \mid X, U_{D}=u_{D}\right)
\end{array}\right) d F_{Z_{a} \mid U_{D}}\left(z \mid u_{D}\right)\right] d F_{U_{D} \mid X}\left(u_{D}\right) \\
& =\int\left[\begin{array}{l}
\left(1-\operatorname{Pr}\left[\mu_{D}\left(Z_{a}\right)<u_{D} \mid U_{D}=u_{D}\right]\right) E\left(Y_{1} \mid X, U_{D}=u_{D}\right) \\
+\operatorname{Pr}\left[\mu_{D}\left(Z_{a}\right)<u_{D} \mid U_{D}=u_{D}\right] E\left(Y_{0} \mid X, U_{D}=u_{D}\right)
\end{array}\right] d F_{U_{D} \mid X}\left(u_{D}\right)
\end{aligned}
$$

where the first equality follows from the law of iterated expectations; the second equality follows by plugging in our model for $D$; the third equality follows from independence $Z \Perp\left(Y_{1}, Y_{0}\right) \mid X, U_{D}$; the fourth equality follows by an application of Fubini's Theorem; and the final equality follows immediately. Thus comparing policy $a$ to policy $a^{\prime}$, we obtain (19) in the text.

Q.E.D.

\section{Proofs of Propositions}

\section{Proof of Proposition 1}

We first show that, given (i), conditions (ii) and (iii) are sufficient for the instrument $J_{x}(Z)$ defined the proposition to have the desired properties. As a preliminary step, note that

$$
E\left(J_{x} \mid X=x\right)=\int_{0}^{1} \mathbf{1}\left[f_{P \mid X}(p \mid x)>0\right] w^{\prime}(p \mid x) d p=\int_{0}^{1} w^{\prime}(p \mid x) d p=0
$$

where the first equality comes from plugging in the proposed $J_{x}$ and using condition (i); the second equality follows from condition (iii); and the final equality follows from condition (ii). We now check that 
the proposed $J_{x}$ is correlated with $D$ under conditions (i) to (iii).

$\operatorname{Cov}\left(J_{x}(Z), D \mid X=x\right)=\operatorname{Cov}\left(J_{x}(Z), P(Z) \mid X=x\right)=\int_{0}^{1} \mathbf{1}\left[f_{P \mid X}(p \mid x)>0\right] w^{\prime}(p \mid x) p d p=\int_{0}^{1} w^{\prime}(p \mid x) p d p=-1$

where the first equality follows from the law of iterated expectations; the second equality comes from plugging in the proposed $J_{x}$ and using $E\left(J_{x} \mid X=x\right)=0$; the third equality uses condition (iii) and the final equality follows from integration by parts using condition (ii). We now check that the proposed instrument $J_{x}$ implies the desired weights on $\Delta^{M T E}$. With the proposed $J_{x}$, for $u$ such that $f_{P \mid X}(u \mid x)>0$,

$$
-\frac{T\left(u \mid x ; J_{x}\right) f_{P \mid X}(u \mid x)}{\operatorname{Cov}\left(J_{x}, P \mid X=x\right)}=w^{\prime}(u \mid x)
$$

where the equality comes from plugging in the proposed $J_{x}$ and using $E\left(J_{x} \mid X=x\right)=0$ and $\operatorname{Cov}\left(J_{x}, P \mid\right.$ $X=x)=-1$. Thus, for $u$ such that $f_{P \mid X}(u \mid x)>0$, we have that $-T\left(u \mid x ; J_{x}\right) f_{P \mid X}(u \mid x) / \operatorname{Cov}\left(J_{x}, P \mid\right.$ $X=x)=w^{\prime}(u \mid x)$ as desired. For $u$ such that $f_{P \mid X}(u \mid x)=0$, condition (iii) implies that $w^{\prime}(u \mid x)=0$, and thus trivially $-T\left(u \mid x ; J_{x}\right) f_{P \mid X}(u \mid x) / \operatorname{Cov}\left(J_{x}, P \mid X=x\right)=w^{\prime}(u \mid x)$ for $u$ such that $f_{P \mid X}(u \mid x)=0$.

We now show that, given condition (i), conditions (ii) and (iii) are necessary. First, consider condition (ii). We have previously established that the weights corresponding to any instrument must integrate to one, and that the weights corresponding to any instrument must satisfy $w(0 \mid x)=0$. One can also directly verify that $w(1 \mid x)=0$ unless the conditional distribution of $P(Z)$ has a mass point at 1 . The conditional distribution of $P(Z)$ does not have a mass point at 1 by condition (i). Using condition (i), one can apply Lebesgue's theorem for the derivative of an integral to show that the weights corresponding to any instrument will be differentiable. Thus, given condition (i), the weights corresponding to any instrument will satisfy condition (ii), and thus condition (ii) is a necessary condition for there to exist an instrument that corresponds to the desired weights. Now assume conditions (i) and (ii), and consider condition (iii). Suppose that (iii) does not hold, so that there exists a set of $t$ values such that $f_{P \mid X}(t \mid x)=0$ but $w^{\prime}(t \mid x)>0$. Then, for such values of $t$,

$$
-\frac{T\left(t \mid x ; J_{x}\right) f_{P \mid X}(t \mid x)}{\operatorname{Cov}\left(J_{x}, P \mid X=x\right)}=0
$$

for any potential instrument $J_{x}$ while $w^{\prime}(t \mid x)>0$, and thus trivially there cannot exist an instrument $J_{x}$ 
such that

$$
-\frac{T\left(t \mid x ; J_{x}\right) f_{P \mid X}(t \mid x)}{\operatorname{Cov}\left(J_{x}, P \mid X=x\right)}=w^{\prime}(t \mid x)
$$

for all $t$. Thus, given condition (i), conditions (ii) and (iii) are necessary for the existence of an instrument with the desired properties.

\section{Proof of Proposition 2}

Define

$$
w(\cdot \mid x) \equiv \frac{F_{P_{a^{\prime}} \mid X}(\cdot \mid x)-F_{P_{a} \mid X}(\cdot \mid x)}{E\left(P_{a} \mid X=x\right)-E\left(P_{a^{\prime}} \mid X=x\right)} .
$$

We now show that conditions (i) and (ii) of Proposition 2 imply conditions (i) and (ii) of Proposition 1 when $w(\cdot \mid x)$ is defined in this manner. Note that condition (i) of Proposition 2 immediately implies that condition (i) of Proposition 1 holds. Condition (i) of Proposition 2 implies that $w(\cdot \mid x)$ is differentiable for all evaluation points with

$$
w^{\prime}(\cdot \mid x)=\frac{f_{P_{a^{\prime}} \mid X}(\cdot \mid x)-f_{P_{a} \mid X}(\cdot \mid x)}{E\left(P_{a} \mid X=x\right)-E\left(P_{a^{\prime}} \mid X=x\right)} .
$$

Since $F_{P_{a} \mid X}(\cdot)$ and $F_{P_{a^{\prime}} \mid X}(\cdot)$ are distribution functions, one can directly verify that $\int_{0}^{1} w(u \mid x) d u=1$. Since the propensity score is always bounded by 0 and 1 , using condition (i), $F_{P_{a} \mid X}(1 \mid x)=F_{P_{a^{\prime}} \mid X}(1 \mid x)=1$ and $F_{P_{a} \mid X}(0 \mid x)=F_{P_{a^{\prime}} \mid X}(0 \mid x)=0$ and thus $w(1 \mid x)=w(0 \mid x)=0$. Defining $w(\cdot \mid x)$ in this manner, we have that conditions (i) and (ii) of Proposition 2 imply conditions (i) and (ii) of Proposition 1. Given

$$
w^{\prime}(\cdot \mid x)=\frac{f_{P_{a^{\prime}} \mid X}(\cdot \mid x)-f_{P_{a} \mid X}(\cdot \mid x)}{E\left(P_{a} \mid X=x\right)-E\left(P_{a^{\prime}} \mid X=x\right)},
$$

we have that condition (iii) of Proposition 2 is equivalent to condition (iii) of Proposition 1 for this choice of $w(\cdot \mid x)$. The result now follows directly from Proposition 1.

Q.E.D.

\section{Proof of Proposition 3}

Assume that the conditions of Proposition 1 hold almost everywhere with respect to $X$. From the proof of Proposition 1, under the stated conditions, $E(J(Z) \mid X)=0, \operatorname{Cov}(J(Z), D \mid X)=-1$, and

$$
\frac{\operatorname{Cov}(J(Z), Y \mid X)}{\operatorname{Cov}(J(Z), D \mid X)}=\int \Delta^{M T E}(X, u) w(u \mid X) d u
$$

It follows that $\operatorname{Cov}(J(Z), D \mid X)=\operatorname{Cov}(J(Z), D \mid X)=-1$, that $\operatorname{Cov}(J(Z), Y)=E(J(Z) Y)=$ 
$E[E(J(Z) Y \mid X)]$, and thus that

$$
\begin{aligned}
\frac{\operatorname{Cov}(J(Z), Y)}{\operatorname{Cov}(J(Z), D)} & =E[-E(J(Z) Y \mid X)] \\
& =E\left[\frac{\operatorname{Cov}(J(Z), Y \mid X)}{\operatorname{Cov}(J(Z), D \mid X)}\right]=\int\left[\int_{0}^{1} \Delta^{M T E}(x, u) w(u \mid x) d u\right] d F_{X}(x) .
\end{aligned}
$$

Q.E.D.

\section{Proof of Proposition 4}

We first show that, given condition (i), conditions (ii) and (iii) are sufficient for the instrument $J_{x}(Z)$ defined by the proposition to have the desired properties. For notational convenience, define $\pi_{l}=\operatorname{Pr}\left[P(Z)=p_{l}\right]$, for $l=1, \ldots, K$. As a preliminary step, note that with this definition of $J_{x}(Z)$,

$$
E\left(J_{x} \mid X=x\right)=\sum_{l=1}^{K} \frac{1}{\pi_{l}}\left[w_{l}-w_{l+1}\right] \pi_{l}=\sum_{l=1}^{K}\left[w_{l}-w_{l+1}\right]=w_{1}-w_{K+1}=0
$$

where we use the fact that $w_{1}=w_{K+1}=0$. We now check that the proposed $J_{x}$ is correlated with $D$ under conditions (i) to (iii).

$$
\begin{aligned}
\operatorname{Cov}\left(J_{x}(Z), D \mid X=x\right) & =\operatorname{Cov}\left(J_{x}(Z), P(Z) \mid X=x\right) \\
& =\sum_{l=1}^{K} \frac{1}{\pi_{l}}\left[w_{l}-w_{l+1}\right] p_{l} \pi_{l}=\sum_{l=2}^{K}\left[p_{l}-p_{l-1}\right] w_{l} \\
& =\sum_{l=1}^{K} \int_{p_{l-1}}^{p_{l}} w(t \mid x) d t=\int_{0}^{1} w(t \mid x) d t=1
\end{aligned}
$$

where the first equality follows from the law of iterated expectations; the second equality comes from $E\left(J_{x} \mid X=x\right)=0$ and plugging in the proposed $J_{x}(Z)$; the third equality rearranges terms in the sum using $w_{1}=w_{K+1}=0$; the fourth equality uses condition (iii) and the definition of $w_{l}$; the fifth equality uses linearity of integration; and the final equality uses condition (ii). We now check that the proposed instrument $J_{x}$ implies the desired weights on $\Delta^{M T E}$. Using $\operatorname{Cov}\left(J_{x}(Z), D \mid X=x\right)=1$ and that $E\left(J_{x}(Z) \mid\right.$ $X=x)=0$, the $I V$ weights corresponding to the proposed $J_{x}(Z)$ as given by $h_{I V}(u)=E\left(J_{x}(Z) \mathbf{1}[P(Z) \geq\right.$

$u]$ ). We immediately have $h_{I V}(u)=w(u \mid x)=0$ for $u \in\left(p_{K}, 1\right]$ and for $u \in\left[0, p_{1}\right]$. For $u \in\left(p_{j-1}, p_{j}\right]$, 
$j=2, \ldots, K$, we have

$$
E\left(J_{x}(Z) \mathbf{1}[P(Z) \geq u]\right)=\sum_{l=1}^{K} \frac{1}{\pi_{l}}\left[w_{l}-w_{l+1}\right] \mathbf{1}\left[p_{l} \geq u\right] \pi_{l}=\sum_{l=j}^{K}\left[w_{l}-w_{l+1}\right]=w_{j}-w_{K+1}=w_{j}=w(u \mid x)
$$

where the first equality comes from plugging in the proposed $J_{x}(Z)$; the second equality follows from $u \in\left(p_{j-1}, p_{j}\right]$; the third equality follows by rearranging terms; the fourth equality follows from $w_{K+1}=0$; and the final equality follows by the definition of $w_{j}$ and the fact that $u \in\left(p_{j-1}, p_{j}\right]$.

We now show that, given condition (i), conditions (ii) and (iii) are necessary. First, consider condition (ii). One can verify that the weights corresponding to any instrument must integrate to one. One can also verify that the weights corresponding to any instrument must satisfy $w(u \mid x)=0$ for $u \leq p_{x}^{\text {Min }}$ and for $u>p_{x}^{M a x}$, where $p_{x}^{M i n}$ and $p_{x}^{M a x}$ are the minimum and maximum values in the support of the conditional distribution of $P(Z)$. Given condition (i), one can immediately verify that the $I V$ weights for any instrument must satisfy condition (iii). Thus, given condition (i), the weights corresponding to any instrument will satisfy conditions (ii) and (iii), and thus, given condition (i), conditions (ii) and (iii) are necessary conditions for there to exist an instrument that corresponds to the desired weights.

Q.E.D.

\section{Local Instrumental Variables for the Random Coefficient Model}

Consider the model:

$$
D=\mathbf{1}[Z \beta \geq 0]
$$

where $\beta$ is a random variable. For ease of exposition, we leave implicit the conditioning on $X$ covariates. Assume that $\left(Y_{0}, Y_{1}, \beta\right) \Perp Z$. Assume that $\beta$ has a density that is absolutely continuous with respect to Lebesgue measure on $\Re^{K}$. We have

$$
E(Y \mid Z=z)=E\left(D Y_{1} \mid Z=z\right)+E\left((1-D) Y_{0} \mid Z=z\right)
$$


To simplify the exposition, first consider the first term, $E\left(D Y_{1} \mid Z=z\right)$. Using the model, the independence assumption, and the law of iterated expectations, we have

$$
\begin{aligned}
E(D Y \mid Z=z) & =E\left(\mathbf{1}[z \beta \geq 0] Y_{1}\right)=E\left(\mathbf{1}[z \beta \geq 0] E\left(Y_{1} \mid \beta\right)\right) \\
& =E\left(\mathbf{1}\left\{z^{[K]} \beta^{[K]} \geq-z^{[-K]} \beta^{[-K]}\right\} E\left(Y_{1} \mid \beta\right)\right)
\end{aligned}
$$

where the final outer expectation is over $\beta$. Consider taking the derivative with respect to the $K$ th element of $Z$ assumed to be continuous. Let $Z^{[K]}$ denote the $K$ th element of $Z$ and $Z^{[-K]}$ denote all other elements of $Z$, and write $Z=\left(Z^{[-K]}, Z^{[K]}\right)$. Likewise, partition $z, \beta$, and $b$ as $z=\left(z^{[-K]}, z^{[K]}\right), \beta=\left(\beta^{[-K]}, \beta^{[K]}\right)$, and $b=\left(b^{[-K]}, b^{[K]}\right)$, where $z$ is a realization of $Z$ and $b$ is a realization of $\beta$. For simplicity, suppose that the $K$ th element of $z$ is positive, $z^{[K]}>0$. We obtain

$$
\begin{aligned}
E(D Y \mid Z=z) & =E\left[E\left(\mathbf{1}\left\{z^{[K]} \beta^{[K]} \geq-z^{[-K]} \beta^{[-K]}\right\} E\left(Y_{1} \mid \beta\right) \mid \beta^{[-K]}\right)\right] \\
& =E\left[E\left(\mathbf{1}\left\{\beta^{[K]} \geq \frac{-z^{[-K]} \beta^{[-K]}}{z^{[K]}}\right\} E\left(Y_{1} \mid \beta\right) \mid \beta^{[-K]}\right)\right],
\end{aligned}
$$

where the inside expectation is over $\beta^{[K]}$ conditional on $\beta^{[-K]}$, i.e., is over the $K$ th element of $\beta$ conditional on all other components of $\beta$. Thus,

$$
\frac{\partial}{\partial z^{[K]}} E(D Y \mid Z=z)=\int E\left(Y_{1} \mid \beta=M\left(b^{[-K]}\right)\right) \tilde{w}\left(b^{[-K]}\right) d b^{[-K]}
$$

where

$$
M\left(b^{[-K]}\right)=\left(\left(b^{[-K]}\right)^{\prime}, \frac{-z^{[-K]} b^{[-K]}}{z^{[K]}}\right)^{\prime} \text { and } \tilde{w}\left(b^{[-K]}\right)=\frac{z^{[-K]} b^{[-K]}}{\left(z^{[K]}\right)^{2}} f\left(b^{[-K]}, \frac{-z^{[-K]} b^{[-K]}}{z^{[K]}}\right)
$$

with $f(\cdot)$ the density of $\beta$ (with respect to Lebesgue measure), and where for notational simplicity we suppress the dependence of the function $M(\cdot)$ and the weights $\tilde{w}(\cdot)$ on the $z$ evaluation point. In this expression, we are averaging over $E\left(Y_{1} \mid \beta=b\right)$, but only over $b$ evaluation points such that $z b=0$. In particular, the expression average over the $K-1$ space of $b^{[-K]}$, while for each potential realization of $b^{[-K]}$ it is filling in the value of $b^{[K]}$ such that $z^{[K]} b^{[K]}=-z^{[-K]} b^{[-K]}$ so that $z^{[K]} b^{[K]}+z^{[-K]} b^{[-K]}=0$. Note that the weights $\tilde{w}\left(b^{-K]}\right)$ will be zero for any $b^{[-K]}$ such that $f\left(b^{[-K]}, \frac{-z^{[-K]} b^{[-K]}}{z^{[K]}}\right)=0$, i.e., the weights will be zero for 
any $b^{[-K]}$ such that there does not exist $b^{[K]}$ in the conditional support of $\beta^{[K]}$ with $z^{[K]} b^{[K]}=-z^{[-K]} b^{[-K]}$.

Following the same logic for $E\left((1-D) Y_{0} \mid Z=z\right)$ we obtain

$$
\frac{\partial}{\partial z^{[K]}} E((1-D) Y \mid Z=z)=-\int E\left(Y_{0} \mid \beta=M\left(b^{[-K]}\right)\right) \tilde{w}\left(b^{[-K]}\right) d b^{[-K]}
$$

and likewise have

$$
\frac{\partial}{\partial z^{[K]}} \operatorname{Pr}(D=1 \mid Z=z)=\int \tilde{w}\left(b^{[-K]}\right) d b^{[-K]}
$$

so that

$$
\frac{\frac{\partial}{\partial z\left[^{K]}\right.} E(Y \mid Z=z)}{\frac{\partial}{\partial z^{[K]}} \operatorname{Pr}(D=1 \mid Z=z)}=\int E\left(Y_{1}-Y_{0} \mid \beta=M\left(b^{[-K]}\right)\right) w\left(b^{[-K]}\right) d b^{[-K]},
$$

where

$$
w\left(b^{[-K]}\right)=\tilde{w}\left(b^{[-K]}\right) / \int \tilde{w}\left(b^{[-K]}\right) d b^{[-K]} .
$$

Now consider the question of whether this expression will include both positive and negative weights. Recall that $\tilde{w}\left(b^{[-K]}\right)=\frac{z^{[-K]} b^{[-K]}}{\left(z^{[K]}\right)^{2}} f\left(b^{[-K]}, \frac{-z^{[-K]}[-K]}{z^{[K]}}\right)$. Thus,

$$
\tilde{w}\left(b^{[-K]}\right) \geq 0 \quad \text { if } z^{[-K]} b^{[-K]}>0, \quad \tilde{w}\left(b^{[-K]}\right) \leq 0 \quad \text { if } z^{[-K]} b^{[-K]}<0,
$$

and will be nonzero if $z^{[-K]} b^{[-K]} \neq 0$ and there exists $b^{[K]}$ in the conditional support of $\beta^{[K]}$ with $z^{[K]} b^{[K]}=$ $z^{[-K]} b^{[-K]}$, i.e., with $z b=0$. We thus have that there will be both positive and negative weights on the $M T E$ if there exists values of $b$ in the support of $\beta$ with both $z^{[-K]} b^{[-K]}>0$ and $z b=0$, and there exists other values of $b$ in the support of $\beta$ with $z^{[-K]} b^{[-K]}<0$ and $z b=0$. 


\section{References}

Ahn, H., And J. Powell (1993): "Semiparametric Estimation of Censored Selection Models with a Nonparametric Selection Mechanism," Journal of Econometrics, 58(1-2), 3-29.

Amemiya, T. (1985): Advanced Econometrics. Harvard University Press, Cambridge, MA.

Andrews, D. W., And M. M. Schafgans (1998): "Semiparametric Estimation of the Intercept of a Sample Selection Model," Review of Economic Studies, 65(3), 497-517.

Angrist, J., K. Graddy, and G. Imbens (2000): "The Interpretation of Instrumental Variables

Estimators in Simultaneous Equations Models with an Application to the Demand for Fish," Review of Economic Studies, 67(3), 499-527.

Angrist, J. D., And A. B. Krueger (1999): "Empirical Strategies in Labor Economics," in Handbook of Labor Economics, ed. by O. Ashenfelter, and D. Card, vol. 3A, pp. 1277-1366. North-Holland, New York.

Buörklund, A., And R. Moffitt (1987): "The Estimation of Wage Gains and Welfare Gains in SelfSelection," Review of Economics and Statistics, 69(1), 42-49.

Campbell, D. T., And J. C. Stanley (1966): Experimental and quasi-experimental designs for research. Rand McNally, Skokie.

Carneiro, P., K. Hansen, and J. J. Heckman (2003): "Estimating Distributions of Treatment Effects with an Application to the Returns to Schooling and Measurement of the Effects of Uncertainty on College Choice," International Economic Review, 44(2), 361-422, 2001 Lawrence R. Klein Lecture.

Chen, X., And Y. FAn (1999): “Consistent Hypothesis Testing in Semiparametric and Nonparametric Models for Econometric Time Series," Journal of Econometrics, 91(2), 373-401.

Cunha, F., J. J. Heckman, And S. Navarro (2005): "Separating Uncertainty from Heterogeneity in Life Cycle Earnings, The 2004 Hicks Lecture," Oxford Economic Papers, 57(191-261), 1-72.

Ellison, G., And S. F. Ellison (1999): "A Simple Framework for Nonparametric Specification Testing," Journal of Econometrics, 96, 1-23. 
Florens, J.-P., J. J. Heckman, C. Meghir, and E. Vytlacil (2002): "Instrumental Variables, Local Instrumental Variables and Control Functions," Discussion Paper CWP15/02, CEMMAP.

Ghosal, S., A. Sen, and A. W. Van Der VaArt (2000): "Testing Monotonicity of Regression," The Annals of Statistics, 28(4), 1054-1082.

GiLl, R. D., And J. M. Robins (2001): "Graphical Models and Causal Inference - Causal Inference for Complex Longitudinal Data: The Continuous Case," Annals of Statistics, 29(6), 1-27.

Hansen, L. P., And T. J. SARgent (1981): "Rational Expectations Models of Dynamically Interrelated Variables," in Linear Rational Expectations Models of Dynamically Interrelated Variables, ed. by R. Lucas, and T. Sargent, pp. 127-156. University of Minnesota Press, Minneapolis.

Heckman, J. J. (1976): "The Common Structure of Statistical Models of Truncation, Sample Selection and Limited Dependent Variables and a Simple Estimator for Such Models," Annals of Economic and Social Measurement, 5(4), 475-492.

(1990): "Varieties of Selection Bias," American Economic Review, 80(2), 313-18.

(1997): "Instrumental Variables: A Study of Implicit Behavioral Assumptions Used in Making Program Evaluations," Journal of Human Resources, 32(3), 441-62, Addendum published vol. 33 no. 1 (Winter 1998).

(2001): "Micro Data, Heterogeneity, and the Evaluation of Public Policy: Nobel Lecture," Journal of Political Economy, 109(4), 673-748.

Heckman, J. J., H. Ichimura, J. Smith, And P. E. Todd (1998): "Characterizing Selection Bias Using Experimental Data," Econometrica, 66(5), 1017-1098.

Heckman, J. J., H. IChimura, And P. E. Todd (1997): "Matching as an Econometric Evaluation Estimator: Evidence from Evaluating a Job Training Programme," Review of Economic Studies, 64(4), $605-654$. 
Heckman, J. J., R. J. LaLonde, and J. A. Smith (1999): "The Economics and Econometrics of Active Labor Market Programs," in Handbook of Labor Economics, ed. by O. Ashenfelter, and D. Card, vol. 3A, chap. 31, pp. 1865-2097. North-Holland, New York.

Heckman, J. J., L. Lochner, And C. TABer (1998): "General-Equilibrium Treatment Effects: A Study of Tuition Policy," American Economic Review, 88(2), 381-86.

Heckman, J. J., and S. Navarro (2004): "Using Matching, Instrumental Variables, and Control Functions to Estimate Economic Choice Models," Review of Economics and Statistics, 86(1), 30-57.

HeCKMan, J. J., And R. RoBB (1985): "Alternative Methods for Evaluating the Impact of Interventions," in Longitudinal Analysis of Labor Market Data, ed. by J. Heckman, and B. Singer, vol. 10, pp. 156-245. Cambridge University Press, New York.

(1986): "Alternative Methods for Solving the Problem of Selection Bias in Evaluating the Impact of Treatments on Outcomes," in Drawing Inferences from Self-Selected Samples, ed. by H. Wainer. Springer-Verlag, New York.

Heckman, J. J., J. Smith, and C. Taber (1998): “Accounting for Dropouts in Evaluations of Social Programs," Review of Economics and Statistics, 80(1), 1-14.

Heckman, J. J., S. Urzua, and E. Vytlacil (2004): "Understanding Instrumental Variables in Models with Essential Heterogeneity," Review of Economics and Statistics Lecture, 2002. Revised.

Heckman, J. J., And E. Vytlacil (1999): "Local Instrumental Variables and Latent Variable Models for Identifying and Bounding Treatment Effects," Proceedings of the National Academy of Sciences, 96, $4730-4734$.

(2001a): "Instrumental Variables, Selection Models, and Tight Bounds on the Average Treatment Effect," in Econometric Evaluation of Labour Market Policies, ed. by M. Lechner, and F. Pfeiffer, pp. 1-15. Center for European Economic Research, New York.

(2001b): "Local Instrumental Variables," in Nonlinear Statistical Modeling: Proceedings of the Thirteenth International Symposium in Economic Theory and Econometrics: Essays in Honor of Takeshi 
Amemiya, ed. by C. Hsiao, K. Morimue, and J. L. Powell, pp. 1-46, New York. Cambridge University Press.

(2005): "Econometric Evaluation of Social Programs," in Handbook of Econometrics, Volume 6, ed. by J. Heckman, and E. Leamer, p. forthcoming. Elsevier, Amsterdam.

Hendry, D. (1995): Dynamic Econometrics. Oxford University Press, New York.

Hurwicz, L. (1962): "On the Structural Form of Interdependent Systems," in Logic, Methodology and Philosophy of Science, ed. by E. Nagel, S. P., and A. Tarski. Stanford University Press.

IChimura, H., And C. TABER (2002): "Semiparametric Reduced-Form Estimation of Tuition Subsidies," American Economic Review, 92(2), 286-92.

IChimura, H., And T. S. Thompson (1998): "Maximum Likelihood Estimation of a Binary Choice Model with Random Coefficients of Unknown Distribution," Journal of Econometrics, 86(2), 269-95.

Imbens, G. W., And J. D. Angrist (1994): "Identification and Estimation of Local Average Treatment Effects," Econometrica, 62(2), 467-75.

Imbens, G. W., And D. B. Rubin (1997): "Estimating Outcome Distributions for Compliers in Instrumental Variables Models," Review of Economic Studies, 64(4), 555-74.

Manski, C. F. (1990): "Nonparametric Bounds on Treatment Effects," American Economic Review, $80(2), 319-23$.

Marschak, J. (1953): "Economic Measurements for Policy and Prediction," in Studies in Econometric Method, ed. by W. Hood, and T. Koopmans, pp. 1-26. Wiley, New York.

Matzkin, R. (1994): "Restrictions of Economic Theory in Nonparametric Methods," in Handbook of Econometrics, ed. by R. Engle, and D. McFadden, vol. 4, pp. 2523-58. North-Holland, New York.

Pearl, J. (2000): Causality. Cambridge University Press, Cambridge, England.

Powell, J. L. (1994): "Estimation of Semiparametric Models," in Handbook of Econometrics, Volume 4, ed. by R. Engle, and D. McFadden, pp. 2443-2521. Elsevier, Amsterdam. 
Roy, A. (1951): "Some Thoughts on the Distribution of Earnings," Oxford Economic Papers, 3(2), 135146.

Rudin, W. (1974): Real and Complex Analysis. McGraw-Hill, New York, second edn.

Smith, V. K., and H. S. Banzhaf (2004): "A Diagrammatic Exposition of Weak Complementarity and the Willig Condition," American Journal of Agricultural Economics, 86(2), 455-66.

Vytlacil, E. (2002): "Independence, Monotonicity, and Latent Index Models: An Equivalence Result," Econometrica, 70(1), 331-41.

(2004): “Additive Separability and Latent Index Models of Binary Choice: Representative Results," Unpublished Paper, Stanford Univerity, Department of Economics.

Yitzhaki, S. (1996): "On Using Linear Regressions in Welfare Economics," Journal of Business and Economic Statistics, 14(4), 478-86.

_ (1999): “The Gini Instrumental Variable or 'The Double IV Estimator',' Unpublished manuscript, Hebrew University, Department of Economics.

Zheng, J. X. (1996): "A Consistent Test of Functional Form via Nonparametric Estimation Techniques," Journal of Econometrics, 75, 263-289. 
Table IA

Treatment Effects and Estimands as Weighted Averages of the Marginal Treatment Effect

$$
\begin{aligned}
& \operatorname{ATE}(x)=\int_{0}^{1} \Delta^{M T E}\left(x, u_{D}\right) d u_{D} \\
& \operatorname{TT}(x)=\int_{0}^{1} \Delta^{M T E}\left(x, u_{D}\right) h_{T T}\left(x, u_{D}\right) d u_{D} \\
& \operatorname{LATE}\left(x, u_{D}, u_{D}^{\prime}\right)=\frac{1}{u_{D}-u_{D}^{\prime}}\left[\int_{u_{D}^{\prime}}^{u_{D}} \Delta^{M T E}(x, u) d u\right] \\
& \operatorname{TUT}(x)=\int_{0}^{1} \Delta^{M T E}\left(x, u_{D}\right) h_{T U T}\left(x, u_{D}\right) d u_{D} \\
& \operatorname{PRTE}(x)=\int_{0}^{1} \Delta^{M T E}\left(x, u_{D}\right) h_{P R T E}\left(x, u_{D}\right) d u_{D} \\
& \operatorname{IV}(x)=\int_{0}^{1} \Delta^{M T E}\left(x, u_{D}\right) h_{I V}\left(x, u_{D}\right) d u_{D} \\
& \operatorname{OLS}(x)=\int_{0}^{1} \Delta^{M T E}\left(x, u_{D}\right) h_{O L S}\left(x, u_{D}\right) d u_{D}
\end{aligned}
$$




\begin{aligned} & \hline \hline$h_{A T E}\left(x, u_{D}\right)=1 \\ & h_{T T}\left(x, u_{D}\right)= {\left[\int_{u_{D}}^{1} f(p \mid X=x) d p\right] \frac{1}{E(P \mid X=x)} } \\ & h_{T U T}\left(x, u_{D}\right)= {\left[\int_{0}^{u_{D}} f(p \mid X=x) d p\right] \frac{1}{E((1-P) \mid X=x)} } \\ & h_{P R T E}\left(x, u_{D}\right)= {\left[\frac{F_{P^{*}, X}\left(u_{D} \mid x\right)-F_{P, X}\left(u_{D} \mid x\right)}{\Delta \bar{P}(x)}\right]$ where $\Delta \bar{P}(x)=E(P \mid X=x)-E\left(P^{*} \mid X=x\right) } \\ & h_{I V}\left(x, u_{D}\right)= {\left[\int_{u_{D}}^{1}(p-E(P \mid X=x)) f(p \mid X=x) d p\right] \frac{1}{\operatorname{Var}(P \mid X=x)}$ for $P(Z)$ as an instrument $} \\ & h_{O L S}\left(x, u_{D}\right)=1+\frac{E\left(U_{1} \mid X=x, U_{D}=u_{D}\right) h_{1}\left(x, u_{D}\right)-E\left(U_{0} \mid X=x, U_{D}=u_{D}\right) h_{0}\left(x, u_{D}\right)}{\Delta^{M T E}\left(x, u_{D}\right)}$, if $\Delta^{M T E}\left(x, u_{D}\right) \neq 0 \\ &,=0$ otherwise \\ &$h_{1}\left(x, u_{D}\right)= {\left[\int_{u_{D}}^{1} f(p \mid X=x) d p\right]\left[\frac{1}{E(P \mid X=x)}\right] } \\ & h_{0}\left(x, u_{D}\right)= {\left[\int_{0}^{u_{D}} f(p \mid X=x) d p\right] \frac{1}{E((1-P) \mid X=x)} } \\ &$\hline\end{aligned}




\section{Table II}

Treatment Parameters and Estimands in the Generalized Roy Example

\begin{tabular}{lr}
\hline \hline Treatment on the Treated & 0.2353 \\
Treatment on the Untreated & 0.1574 \\
Average Treatment Effect & 0.2000 \\
Sorting Gain $^{a}$ & 0.0353 \\
Policy Relevant Treatment Effect $(P R T E)_{\text {Selection Bias }^{b}}$ & 0.1549 \\
Linear Instrumental Variables $^{c}$ & -0.0628 \\
Ordinary Least Squares $^{c}$ & 0.2013 \\
\hline
\end{tabular}

${ }^{a} T T-A T E=E\left(Y_{1}-Y_{0} \mid D=1\right)-E\left(Y_{1}-Y_{0}\right)$

${ }^{b} O L S-T T=E\left(Y_{0} \mid D=1\right)-E\left(Y_{0} \mid D=0\right)$

${ }^{c}$ Using Propensity Score $P(Z)$ as the instrument.

Note: The model used to create Table II is the same as those used to create Figures $1 \mathrm{~A}$ and $1 \mathrm{~B}$. The PRTE is computed using a policy $t$ characterized as follows:

If $Z>0$ then $D=1$ if $Z(1+t)-V>0$.

If $Z \leq t$ then $D=1$ if $Z-V>0$.

For this example $t$ is set equal to 0.2 . 
Table III

Linear Instrumental Variable Estimands and the Policy Relevant Treatment Effect

Using Propensity Score $P(Z)$ as the Instrument 0.2013

Using Propensity Score $P(Z(1+t(\mathbf{1}[Z>0])))$ as the Instrument $\quad 0.1859$

$\begin{array}{ll}\text { Using a dummy } B \text { as an Instrument } & \\ & \end{array}$

Policy Relevant Treatment Effect (PRTE) 0.1549

${ }^{a}$ The dummy $B$ is such that $B=1$ if an individual belongs to a randomly assigned eligible population, 0 otherwise. 
Table IV

The $I V$ Estimator and $\operatorname{Cov}\left(Z_{1}, \beta^{\prime} Z\right)$ Associated with each Value of $\Sigma_{2}$ (Group 2 Covariance)

\begin{tabular}{|c|c|c|c|}
\hline Weights & $\Sigma_{2}$ & $I V$ & $\operatorname{Cov}\left(Z_{1}, \beta^{\prime} Z\right)=\beta^{\prime} \Sigma_{2}^{1}$ \\
\hline$h_{1}$ & $\begin{array}{cc}0.6 & -0.3 \\
-0.3 & 0.6\end{array}$ & 0.133 & -0.30 \\
\hline$h_{2}$ & $\begin{array}{cc}0.6 & -0.1 \\
-0.1 & 0.6\end{array}$ & 0.177 & -0.02 \\
\hline$h_{3}$ & $\left.\begin{array}{ll}0.6 & 0.1 \\
0.1 & 0.6\end{array}\right]$ & 0.194 & 0.26 \\
\hline
\end{tabular}

Weights for Mixture of Normals $I V$ :

$h_{I V}(v)=\frac{\left[\frac{P_{1} \beta^{\prime} \Sigma_{1}^{1}}{\left(\beta^{\prime} \Sigma_{1} \beta\right)^{1 / 2}} \exp \left[-\frac{1}{2}\left(\frac{v-\beta^{\prime} \mu_{1}}{\left(\beta^{\prime} \Sigma_{1} \beta\right)^{1 / 2}}\right)^{2}\right]+\frac{P_{2} \beta^{\prime} \Sigma_{2}^{1}}{\left(\beta^{\prime} \Sigma_{2} \beta\right)^{1 / 2}} \exp \left[-\frac{1}{2}\left(\frac{v-\beta^{\prime} \mu_{2}}{\left(\beta^{\prime} \Sigma_{2} \beta\right)^{1 / 2}}\right)^{2}\right]\right] f_{V}(v)}{\frac{P_{1} \beta^{\prime} \Sigma_{1}^{1}}{\left(\beta^{\prime} \Sigma_{1} \beta+\sigma_{V}^{2}\right)^{1 / 2}} \exp \left[-\left(\frac{-\beta^{\prime} \mu_{1}}{\left(\beta^{\prime} \Sigma_{1} \beta+\sigma_{V}^{2}\right)^{1 / 2}}\right)^{2}\right]+\frac{P_{2} \beta^{\prime} \Sigma_{2}^{1}}{\left(\beta^{\prime} \Sigma_{2} \beta+\sigma_{V}^{2}\right)^{1 / 2}} \exp \left[-\left(\frac{-\beta^{\prime} \mu_{2}}{\left(\beta^{\prime} \Sigma_{2} \beta+\sigma_{V}^{2}\right)^{1 / 2}}\right)^{2}\right]}$,

where $\Sigma_{1}^{1}$ and $\Sigma_{2}^{1}$ are the first rows of $\Sigma_{1}$ and $\Sigma_{2}$, respectively. Clearly, $h_{I V}(-\infty)=0, h_{I V}(\infty)=0$.

The weights clearly integrate to one over the support of $V=(-\infty, \infty)$. Observe that if $P_{2}=0$, the weights must be positive. Thus the structure of the covariances of the instruments is a key determinant of the positivity of the weights for any instrument. It has nothing to do with the ceteris paribus effect of $Z_{1}$ on $P(Z)$ in the general case (changing $Z_{1}$ holding all other components of $Z$ fixed). Now observe that a necessary condition for $h_{I V}<0$ is that sign $\left(\beta^{\prime} \Sigma_{1}^{1}\right)=-\operatorname{sign}\left(\beta^{\prime} \Sigma_{2}^{1}\right)$, i.e., that the covariance between $Z_{1}$ and $\beta^{\prime} Z$ be of opposite signs in the two populations. Without loss of generality assume that $\beta^{\prime} \Sigma_{1}^{1}>0$. If it equals zero, we fail the rank condition. $f_{V}(v)$ is the density of $V$. 
Table V

Comparison of Alternative Approaches to Program Evaluation

\begin{tabular}{|c|c|c|c|}
\hline & $\begin{array}{c}\text { Structural Econometric } \\
\text { Approach }\end{array}$ & $\begin{array}{c}\text { Treatment Effect } \\
\text { Approach }\end{array}$ & $\begin{array}{l}\text { Approach Based } \\
\text { on } M T E\end{array}$ \\
\hline Interpretability & $\begin{array}{l}\text { Well defined economic } \\
\text { parameters and } \\
\text { welfare comparisons }\end{array}$ & $\begin{array}{l}\text { Link to economics } \\
\text { and welfare comparisons } \\
\text { obscure }\end{array}$ & $\begin{array}{l}\text { Interpretable in terms of } \\
\text { willingness to pay; } \\
\text { weighted averages of the } \\
M T E \text { answer well-posed } \\
\text { economic questions }\end{array}$ \\
\hline $\begin{array}{l}\text { Range of Questions } \\
\text { Addressed }\end{array}$ & $\begin{array}{l}\text { Answers many } \\
\text { counterfactual questions }\end{array}$ & $\begin{array}{l}\text { Focuses on one treatment } \\
\text { effect or narrow range } \\
\text { of effects }\end{array}$ & $\begin{array}{l}\text { With support conditions, } \\
\text { generates all } \\
\text { treatment parameters }\end{array}$ \\
\hline $\begin{array}{l}\text { Extrapolation to } \\
\text { New Environments }\end{array}$ & $\begin{array}{l}\text { Provides ingredients for } \\
\text { extrapolation }\end{array}$ & $\begin{array}{l}\text { Evaluates one program in } \\
\text { one environment }\end{array}$ & $\begin{array}{l}\text { Can be partially extrapolated; } \\
\text { extrapolates to new policy environments } \\
\text { with different distributions of the } \\
\text { probability of participation due solely } \\
\text { to differences in distributions of } Z \text {; }\end{array}$ \\
\hline $\begin{array}{l}\text { Comparability } \\
\text { Across Studies }\end{array}$ & $\begin{array}{l}\text { Policy invariant parameters } \\
\text { comparable across studies }\end{array}$ & $\begin{array}{l}\text { Not generally } \\
\text { comparable }\end{array}$ & $\begin{array}{l}\text { Partially comparable; } \\
\text { comparable across environments } \\
\text { with different distributions of the } \\
\text { probability of participation due solely } \\
\text { to differences in distributions of } Z \text {. }\end{array}$ \\
\hline $\begin{array}{l}\text { Key Econometric } \\
\text { Problems }\end{array}$ & $\begin{array}{l}\text { Exogeneity, policy invariance } \\
\text { and selection bias }\end{array}$ & Selection bias & $\begin{array}{l}\text { Selection bias: exogeneity and policy } \\
\text { invariance if used for forecasting }\end{array}$ \\
\hline $\begin{array}{l}\text { Range of Policies } \\
\text { that Can Be } \\
\text { Evaluated }\end{array}$ & $\begin{array}{l}\text { Programs with either partial } \\
\text { or universal coverage, } \\
\text { depending on variation } \\
\text { in data (prices/endowments) }\end{array}$ & $\begin{array}{l}\text { Programs with partial } \\
\text { coverage } \\
\text { (treatment and } \\
\text { control groups) }\end{array}$ & $\begin{array}{l}\text { Programs with partial coverage } \\
\text { (treatment and } \\
\text { control groups) }\end{array}$ \\
\hline $\begin{array}{l}\text { Extension to } \\
\text { General Equilibrium } \\
\text { Evaluation }\end{array}$ & $\begin{array}{l}\text { Need to link to time series data; } \\
\text { parameters compatible with } \\
\text { general equilibrium theory }\end{array}$ & $\begin{array}{l}\text { Difficult because link to } \\
\text { economics is not } \\
\text { precisely specified }\end{array}$ & $\begin{array}{l}\text { Can be linked to nonparametric } \\
\text { general equilibrium models under } \\
\text { exogeneity and policy invariance }\end{array}$ \\
\hline
\end{tabular}


Figure 1A

Weights for the Marginal Treatment Effect for Different Parameters

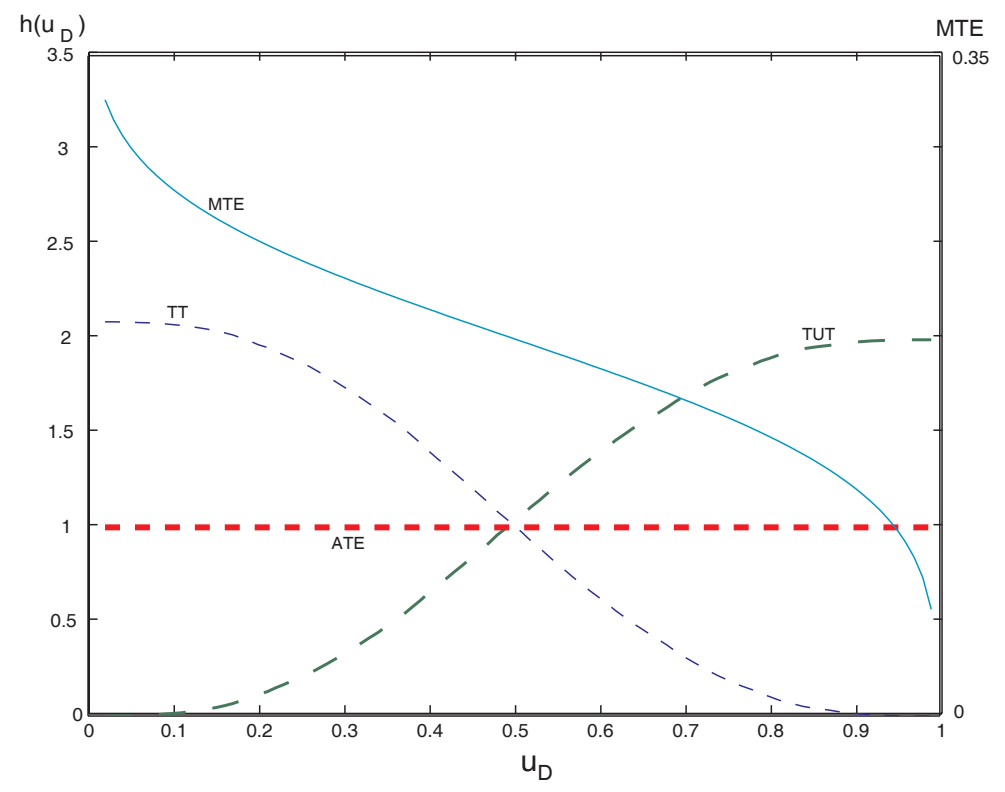

Figure 1B

Marginal Treatment Effect vs Linear Instrumental Variables and Ordinary Least Squares Weights

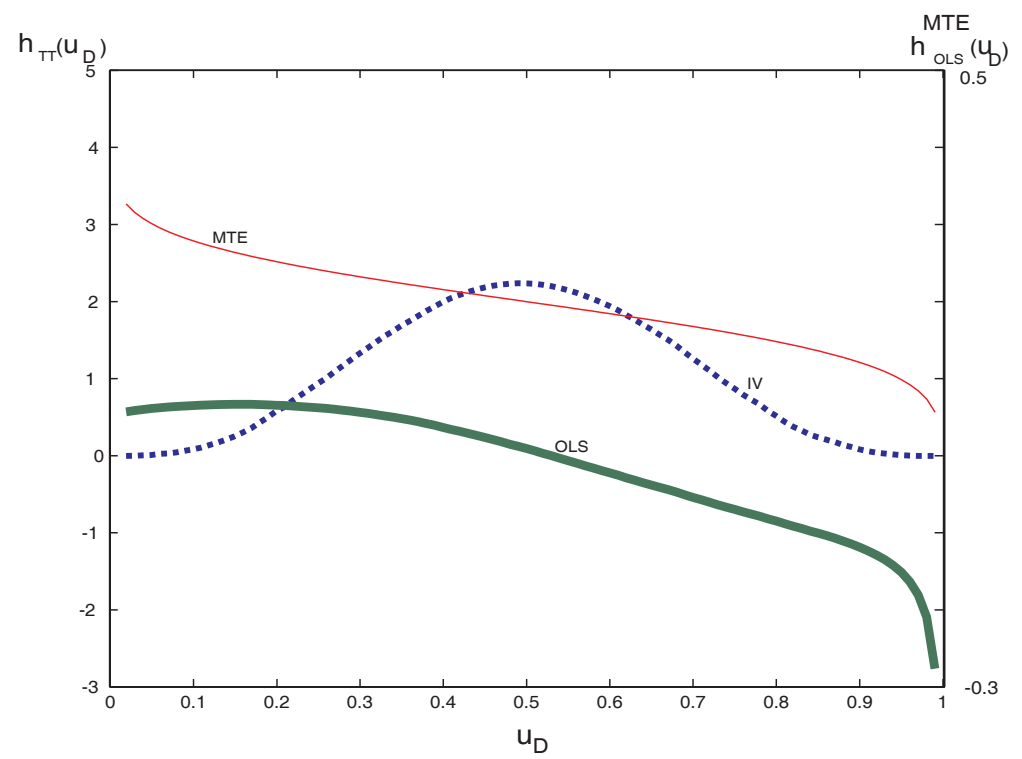

$$
\begin{array}{llll}
Y_{1}=\gamma+\alpha+U_{1} & U_{1}=\sigma_{1} \varepsilon & \gamma=0.67 & \sigma_{1}=0.012 \\
Y_{0}=\gamma+U_{0} & U_{0}=\sigma_{0} \varepsilon & \alpha=0.2 & \sigma_{0}=-0.050 \\
D=1 \text { if } Z-V>0 & V=\sigma_{V} \varepsilon & \varepsilon \sim N(0,1) & \sigma_{V}=-1.000 \\
& U_{D}=\Phi\left(\frac{V}{\sigma_{V} \sigma_{\varepsilon}}\right) & & Z \sim N(-0.0026,0.2700)
\end{array}
$$


Figure 2A

Plot of the $E(Y \mid P(Z)=p)$

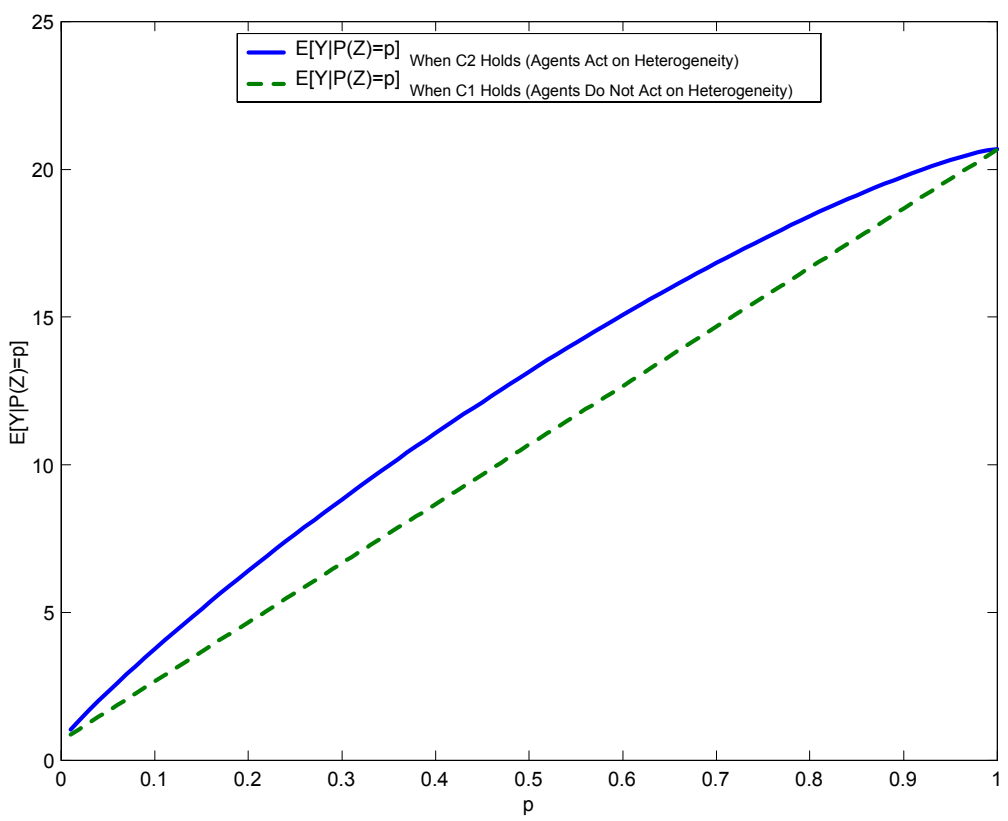

Figure 2B

Plot of the Identified Marginal Treatment Effect from Figure 2A (the Derivative).

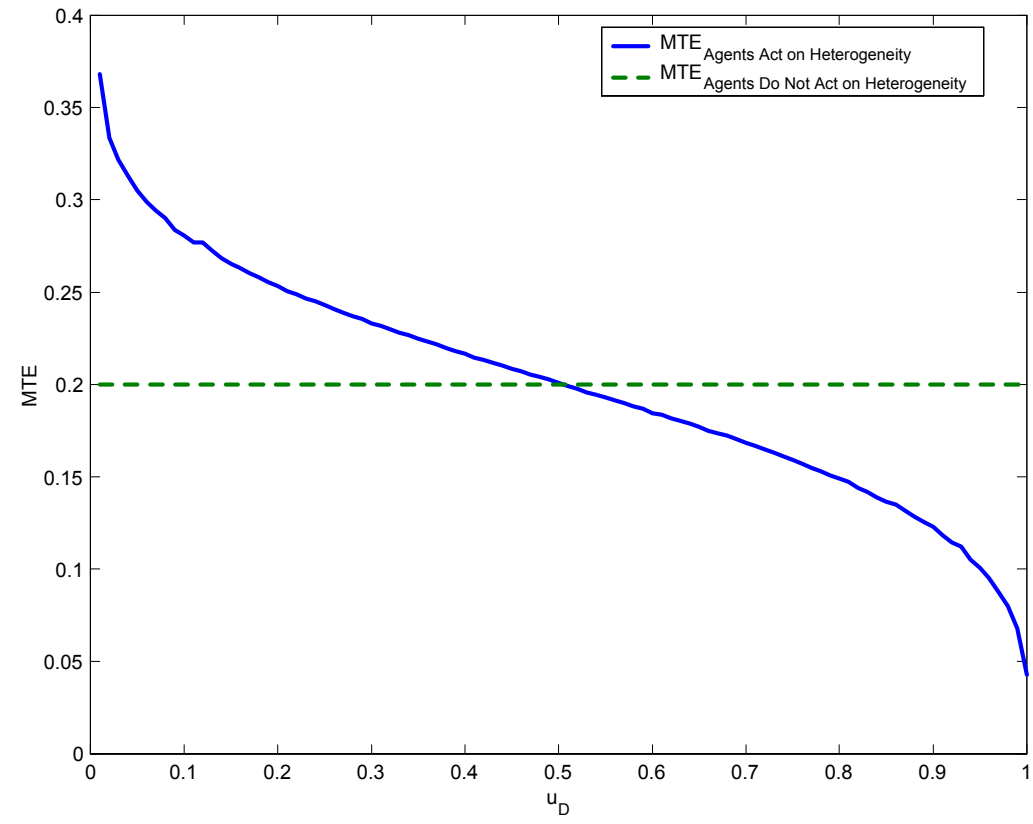

Note: Parameters for the general heterogeneous case are the same as those used in Figures $1 \mathrm{~A}$ and $1 \mathrm{~B}$. For the homogeneous case we impose $U_{1}=U_{0}\left(\sigma_{1}=\sigma_{0}=0.012\right)$. 
Figure 3A

Marginal Treatment Effect vs Linear Instrumental Variables, Ordinary Least Squares, and Policy Relevant Treatment Effect Weights: When $P(Z)$ is the Instrument

The Policy is Given at the Base of Table II

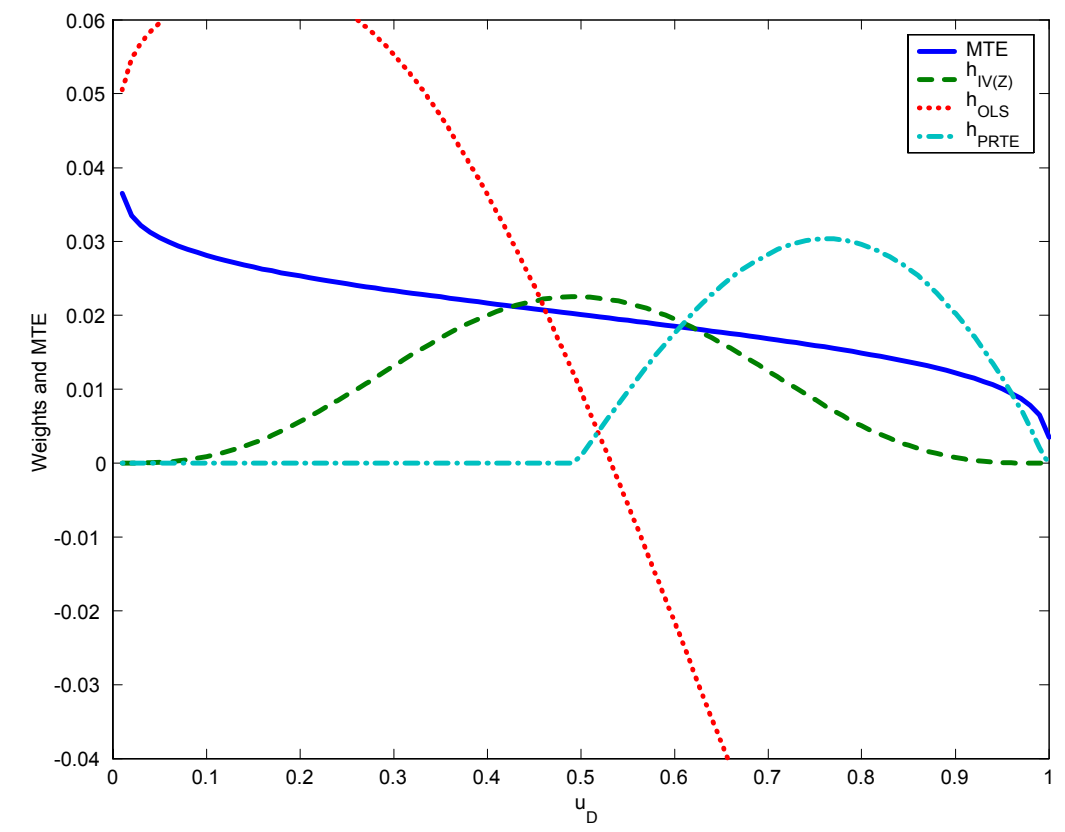

Figure 3B

Marginal Treatment Effect vs Linear $I V$ with $Z$ as an Instrument, Linear $I V$ with $P(Z(1+t(\mathbf{1}[Z>0])))=\tilde{P}(Z, t)$ as an Instrument, and Policy Relevant Treatment Effect Weights

For The Policy Defined at the Base of Table II

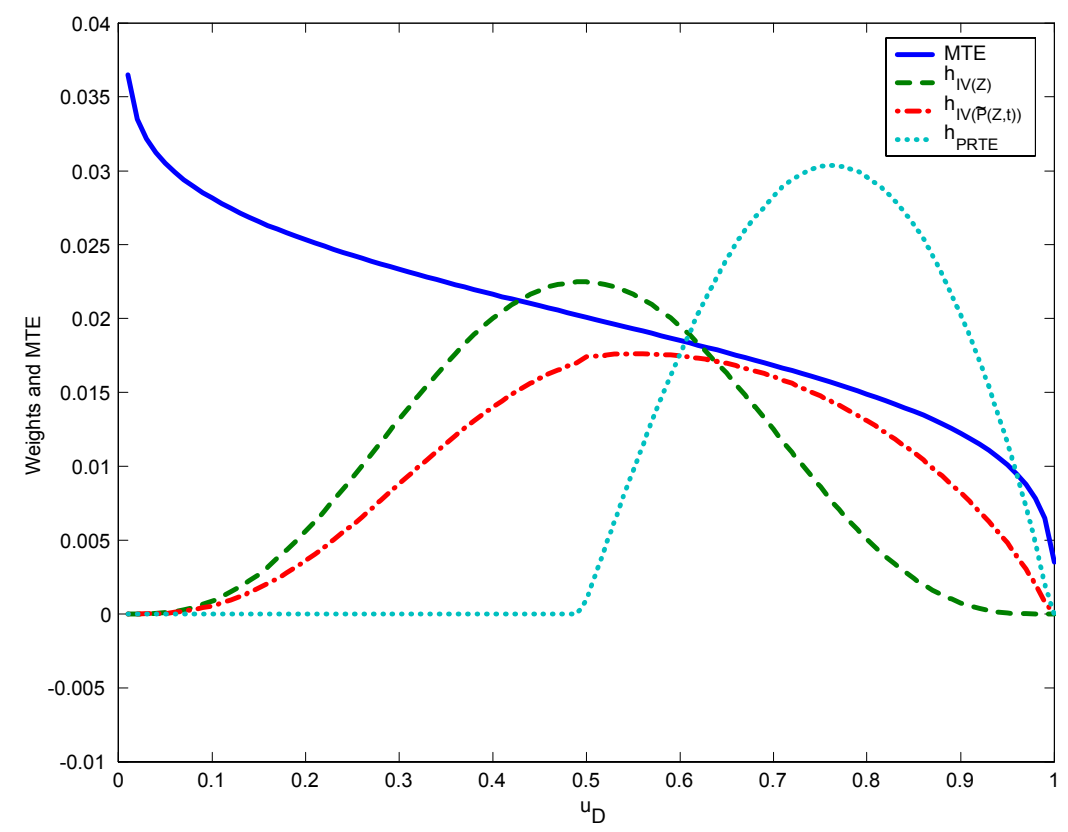


Figure 3C

Marginal Treatment Effect vs $I V$ Policy and Policy Relevant Treatment Effect Weights For The Policy Defined at the Base of Table II

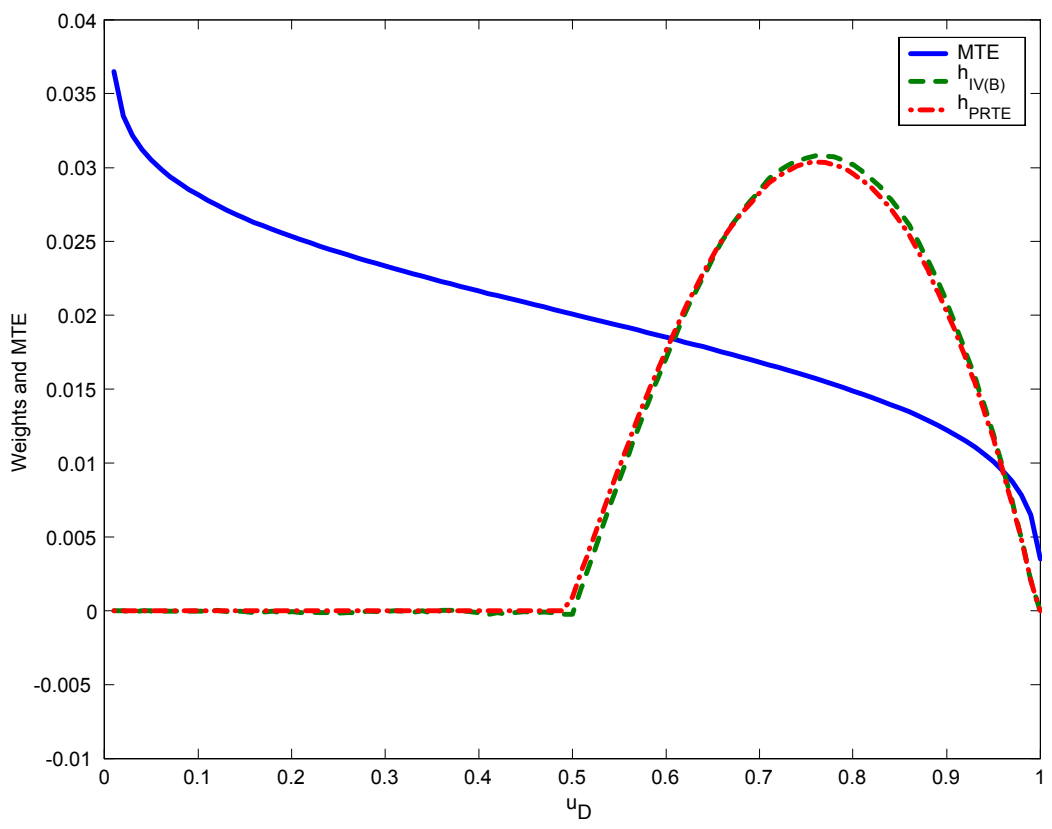


Figure 4

$I V$ Weights when $Z \sim p_{1} N\left(\mu_{1}, \Sigma_{1}\right)+p_{2} N\left(\mu_{2}, \Sigma_{2}\right)$ for Different Values of $\Sigma_{2}$

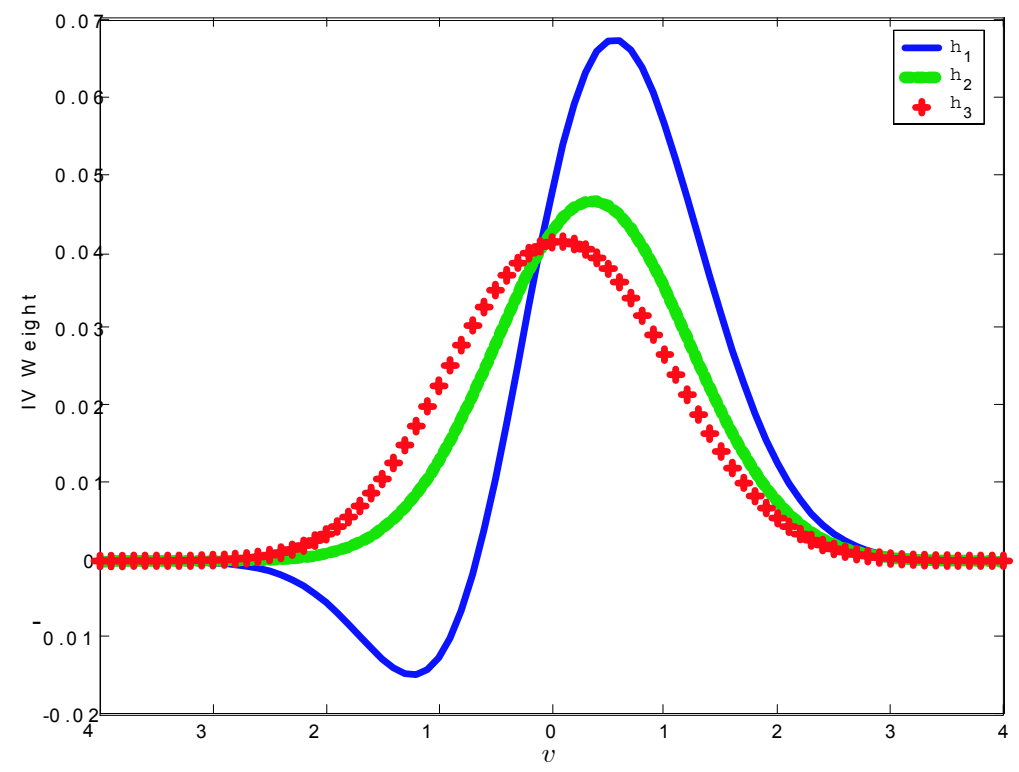

$$
\begin{aligned}
& Y_{1}=\gamma+\alpha+U_{1} \quad U_{1}=\sigma_{1} \epsilon \quad \epsilon \sim N(0,1) \\
& Y_{0}=\gamma+U_{0} \quad U_{0}=\sigma_{0} \epsilon \quad \sigma_{1}=0.012, \sigma_{0}=-0.05, \sigma_{V}=-1 \\
& I=\beta^{\prime} Z-V \quad V=\sigma_{V} \epsilon \quad \gamma=0.67, \alpha=0.2 \\
& D=\left\{\begin{array}{l}
1 \text { if } I>0 \\
0 \text { if } I \leq 0
\end{array}\right. \\
& Z \sim p_{1} N\left(\mu_{1}, \Sigma_{1}\right)+p_{2} N\left(\mu_{2}, \Sigma_{2}\right) \\
& \mu_{1}=\left[\begin{array}{ll}
0 & -1
\end{array}\right], \mu_{2}=\left[\begin{array}{ll}
0 & 1
\end{array}\right] \quad \Sigma_{1}=\left[\begin{array}{ll}
1.4 & 0.5 \\
0.5 & 1.4
\end{array}\right] \\
& p_{1}=0.45, p_{2}=0.55 \quad \beta=\left[\begin{array}{ll}
0.2 & 1.4
\end{array}\right] \\
& \operatorname{Cov}\left(Z_{1}, \beta^{\prime} Z\right)=\beta^{\prime} \Sigma_{1}^{1}=0.98(\text { Group } 1)
\end{aligned}
$$

$$
\begin{aligned}
\Delta^{M T E}(v) & =\alpha+\left[\frac{\operatorname{Cov}\left(U_{1}-U_{0}, V\right)}{\operatorname{Var}(V)}\right] v \\
h_{I V}(v) & =\frac{E\left(Z_{1} \mid \beta^{\prime} Z>v\right) \operatorname{Pr}\left(\beta^{\prime} Z>v\right)}{\operatorname{Cov}\left(Z_{1}, D\right)} \\
\alpha_{I V} & =\int_{-\infty}^{\infty} \Delta^{M T E}(v) h_{I V}(v) d v
\end{aligned}
$$

